\title{
On the Wellposedness of Three-Dimensional Inhomogeneous Navier-Stokes Equations in the Critical Spaces
}

\author{
Hammadi Abidi, Guilong Gui \& Ping Zhang
}

Communicated by V. ŠvERÀK

\begin{abstract}
We prove the local wellposedness of three-dimensional incompressible inhomogeneous Navier-Stokes equations with initial data in the critical Besov spaces, without assumptions of small density variation. Furthermore, if the initial velocity field is small enough in the critical Besov space $\dot{B}_{2,1}^{1 / 2}\left(\mathbb{R}^{3}\right)$, this system has a unique global solution.
\end{abstract}

\section{Introduction}

In this paper, we consider the local and global wellposedness of the following three-dimensional incompressible inhomogeneous Navier-Stokes equations with initial data in the critical Besov spaces:

$$
\left\{\begin{array}{l}
\partial_{t} \rho+\operatorname{div}(\rho u)=0, \quad(t, x) \in \mathbb{R}^{+} \times \mathbb{R}^{3} \\
\partial_{t}(\rho u)+\operatorname{div}(\rho u \otimes u)-\operatorname{div}(\mu \mathcal{M})+\nabla \Pi=0 \\
\operatorname{div} u=0, \\
\left.\rho\right|_{t=0}=\rho_{0},\left.\quad \rho u\right|_{t=0}=m_{0},
\end{array}\right.
$$

where $\rho$ and $u=\left(u_{1}, u_{2}, u_{3}\right)$ stand for the density and velocity of the fluid, respectively, $\mathcal{M}=\frac{1}{2}\left(\partial_{i} u_{j}+\partial_{j} u_{i}\right), \Pi$ is a scalar pressure function, and in general, the viscosity coefficient $\mu(\rho)$ is a smooth, positive function on $[0, \infty)$. This system describes a fluid obtained by mixing two miscible fluids which are incompressible and which have different densities. It can also describe a fluid containing a melted substance. The reader may refer to [20] for the detailed derivation.

KAZHIKOV [18] proved that the inhomogeneous Navier-Stokes equations (1.1) have at least one global weak solution in the energy space when $\mu(\rho)$ is independent of $\rho$, that is, $\mu$ is a positive constant, and $\rho_{0}$ is bounded away from 0 . In addition, he also proved the global existence of strong solutions to this system for small data in three space dimensions and all data in two dimensions. However, the uniqueness 
of both types of weak solutions has not been solved. LADYŽENSKAJA and SOLONNIKOV [19] first addressed the question of unique resolvability of (1.1). More precisely, they considered the system (1.1) in bounded domain $\Omega$ with homogeneous Dirichlet boundary conditions for $u$. Under the assumption that $u_{0} \in W^{2-\frac{2}{p}, p}(\Omega)(p>N)$ is divergence-free and vanishes on $\partial \Omega$, and that $\rho_{0} \in C^{1}(\Omega)$ is bounded away from zero, then they [19] proved

- Global wellposedness in dimension $N=2$;

- Local wellposedness in dimension $N=3$. If in addition $u_{0}$ is small in $W^{2-\frac{2}{p}, p}(\Omega)$, then global wellposedness holds true.

Similar results were obtained by DANCHIN [11] in $\mathbb{R}^{N}$ with initial data in the almost-critical Sobolev spaces.

In general, DiPeRnA and LiONS [15,20] proved the global existence of weak solutions to (1.1) in any spacial dimensions. Yet the uniqueness and regularities of such weak solutions are big open questions even in two space dimensions, as mentioned by Lions [20]. Except under the additional assumptions that

$$
\inf _{c>0}\left\|\frac{\mu\left(\rho_{0}\right)}{c}-1\right\|_{L^{\infty}\left(\mathbb{T}^{2}\right)} \leqq \varepsilon \text { and } u_{0} \in H^{1}\left(\mathbb{T}^{2}\right),
$$

DesJaRDINS [14] proved that $u \in L^{\infty}\left([0, T] ; H^{1}\left(\mathbb{T}^{2}\right)\right)$ and $\rho \in L^{\infty}\left([0, T] \times \mathbb{T}^{2}\right)$ for the weak solution $(\rho, u)$ constructed in [20]. Moreover, with additional regularity assumptions on the initial data, he could also prove that $u \in L^{2}\left([0, \tau] ; H^{2}\left(\mathbb{T}^{2}\right)\right.$ for some short time $\tau$. In [17], the last two authors of the present paper proved that in the two-dimensional case, if the initial density is close enough to a positive constant, then for any given smooth initial velocity field, (1.1) has a unique global smooth solution. Moreover, the velocity $u(t, x)$ decays to 0 with a precise decay rate at $t=\infty$. One may also check [23] for a modified two-dimensional model system of (1.1), where the author proved the global wellposedness of this system with general large initial data. Very recently, we [2] investigated the large time decay and stability of any given global smooth solution of (1.1) for the constant viscosity coefficient case.

On the other hand, if the density $\rho$ is away from zero, which we denote by $a \stackrel{\text { def }}{=} \rho^{-1}-1$ and $\tilde{\mu}(a) \stackrel{\text { def }}{=} \mu(\rho)$, then the system (1.1) can be equivalently reformulated as

$$
(\mathrm{INS}) \quad\left\{\begin{array}{l}
\partial_{t} a+u \cdot \nabla a=0, \quad(t, x) \in \mathbb{R}^{+} \times \mathbb{R}^{N}, \\
\partial_{t} u+u \cdot \nabla u+(1+a)(\nabla \Pi-\operatorname{div}(\tilde{\mu}(a) \mathcal{M}))=0, \\
\operatorname{div} u=0, \\
\left.(a, u)\right|_{t=0}=\left(a_{0}, u_{0}\right) .
\end{array}\right.
$$

Just as the classical Navier-Stokes system, which is the case when $a=0$ in (INS), the system (INS) also has a scaling; indeed if $(a, u)$ solves (INS) with initial data $\left(a_{0}, u_{0}\right)$, then for $\forall \ell>0$,

$$
(a, u)_{\ell}(t, x) \stackrel{\text { def }}{=}\left(a\left(\ell^{2} \cdot, \cdot\right), \ell u\left(\ell^{2} \cdot, \cdot\right)\right)
$$


is also a solution of (INS) with initial data $\left(a_{0}(\ell \cdot), \ell u_{0}(\ell \cdot)\right)$. In particular, the norm of $(a, u) \in \dot{B}_{2, \infty}^{N / 2}\left(\mathbb{R}^{N}\right) \cap L^{\infty}\left(\mathbb{R}^{N}\right) \times \dot{B}_{2,1}^{(N / 2)-1}\left(\mathbb{R}^{N}\right)$ is scaling invariant under this change of scale. In [9], Danchin studied, in general space dimension $N$, the unique solvability of the system (INS) with constant viscosity coefficient and in scaling invariant (or critical) homogeneous Besov spaces, which generalized the celebrated results by FujITA and Kato [16] devoted to the classical Navier-Stokes equations. More precisely, he proved that if the initial data $\left(a_{0}, u_{0}\right) \in \dot{B}_{2, \infty}^{N / 2}\left(\mathbb{R}^{N}\right) \cap L^{\infty}\left(\mathbb{R}^{N}\right) \times$ $\dot{B}_{2,1}^{N / 2-1}\left(\mathbb{R}^{N}\right)$ with $a_{0}$ sufficiently small in $\dot{B}_{2, \infty}^{N / 2}\left(\mathbb{R}^{N}\right) \cap L^{\infty}\left(\mathbb{R}^{N}\right)$, then the system (INS) has a unique local-in-time solution. In [1], Abidi proved that if $1<p<2 N$, $0<\underline{\mu}<\mu(\rho), u_{0} \in \dot{B}_{p, 1}^{N / p-1}\left(\mathbb{R}^{N}\right)$ and $a_{0}=\rho_{0}{ }^{-1}-1 \in \dot{B}_{p, 1}^{N / p}\left(\mathbb{R}^{N}\right)$, then (INS) has a global solution provided that $\left\|a_{0}\right\|_{\dot{B}_{p, 1}^{N / p}}+\left\|u_{0}\right\|_{\dot{B}_{p, 1}^{N / p-1}} \leqq c_{0}$ for $c_{0}$ sufficiently small. Furthermore, the solution thus obtained is unique if $1<p \leqq N$. This result generalized the corresponding results of $[9,11]$ for the constant viscosity case. ABIDI and PAICU [3] further improved the wellposedness results in [1,9] for more general $p$ when $\tilde{\mu}(a)$ equals a positive constant.

In summary, all the wellposedness results of (INS) in the critical spaces obtained so far carry the additional assumption that the initial density is close enough to a positive constant. We should mention that in the very interesting paper [13], Danchin first proved the local wellposedness of compressible isentropic NavierStokes equations in critical Besov spaces, but without the small density variation assumption.

Motivated by [13], we shall investigate the local and global wellposedness of the three-dimensional incompressible inhomogeneous Navier-Stokes equations (1.1) with a constant viscosity coefficient and with initial data in the critical Besov spaces. Compared with [13], the main difficulty of our local wellposedness result, below, lies in the estimate of the pressure term.

For simplicity, we just take $\mu(\rho)=1$ and the space dimension $N=3$. In this case, (INS) becomes

$$
\left\{\begin{array}{l}
\partial_{t} a+u \cdot \nabla a=0, \quad(t, x) \in \mathbb{R}^{+} \times \mathbb{R}^{3}, \\
\partial_{t} u+u \cdot \nabla u+(1+a)(\nabla \Pi-\Delta u)=0 \\
\operatorname{div} u=0 \\
\left.(a, u)\right|_{t=0}=\left(a_{0}, u_{0}\right),
\end{array}\right.
$$

or equivalently

$$
\left\{\begin{array}{l}
\partial_{t} \rho+u \cdot \nabla \rho=0, \quad(t, x) \in \mathbb{R}^{+} \times \mathbb{R}^{3}, \\
\partial_{t}(\rho u)+\operatorname{div}(\rho u \otimes u)-\Delta u+\nabla \Pi=0, \\
\operatorname{div} u=0, \\
\left.(\rho, u)\right|_{t=0}=\left(\rho_{0}, u_{0}\right),
\end{array}\right.
$$

for $\rho=\frac{1}{1+a}$.

Our main result in this paper concerns the unique solvability of (1.2) with initial data in the critical Besov spaces but without a smallness assumption on $a_{0}$. We should mention the method in [13] will be very useful in the proof of Theorem 1, below. 
Theorem 1. Let $a_{0} \in B_{2,1}^{3 / 2}\left(\mathbb{R}^{3}\right), u_{0} \in \dot{B}_{2,1}^{1 / 2}\left(\mathbb{R}^{3}\right)$ with div $u_{0}=0$, and

$$
1+a_{0} \geqq \underline{b}
$$

for some positive constant $\underline{b}$. Then there exists a positive time $T$ such that (1.2) has a unique local-in-time solution $(a, u) \in \mathcal{C}_{b}\left([0, T] ; B_{2,1}^{3 / 2}\left(\mathbb{R}^{3}\right)\right) \times \mathcal{C}_{b}([0, T]$; $\left.\dot{B}_{2,1}^{1 / 2}\left(\mathbb{R}^{3}\right)\right) \cap L^{1}\left([0, T] ; \dot{B}_{2,1}^{5 / 2}\left(\mathbb{R}^{3}\right)\right)$. Moreover, there exits a small constant c depending on $\left\|a_{0}\right\|_{B_{2,1}^{3 / 2}}$ such that if

$$
\left\|u_{0}\right\|_{\dot{B}_{2,1}^{1 / 2}} \leqq c,
$$

(1.2) has a unique global solution $(a, u)$ satisfying

$$
\begin{gathered}
\|a\|_{\widetilde{L}_{t}^{\infty}\left(B_{2,1}^{3 / 2}\right)}+\|u\|_{\widetilde{L}_{t}^{\infty}\left(\dot{B}_{2,1}^{1 / 2}\right)}+\|u\|_{L_{t}^{1}\left(\dot{B}_{2,1}^{5 / 2}\right)}+\|\nabla \Pi\|_{L_{t}^{1}\left(\dot{B}_{2,1}^{1 / 2}\right)} \\
\leqq C\left(\left\|a_{0}\right\|_{B_{2,1}^{3 / 2}}+\left\|u_{0}\right\|_{\left.\dot{B}_{2,1}^{1 / 2}\right)} \exp \{C \sqrt{t}\} \text { for any } t>0 .\right.
\end{gathered}
$$

Scheme of the proof and organization of the paper The main difficulty in proving Theorem 1 lies in the fact that when $a$ is not small, we cannot use the classical arguments in $[1,3,9,11]$ to deal with the following linearized system of (1.2):

$$
\left\{\begin{array}{l}
\partial_{t} u-(1+a)(\Delta u-\nabla \Pi)=f \\
\operatorname{div} u=0 \\
\left.u\right|_{t=0}=u_{0}
\end{array}\right.
$$

Motivated by [13], for some large enough integer $m$, we shall rewrite (1.6) as

$$
\left\{\begin{array}{l}
\partial_{t} u-\left(1+S_{m} a\right)(\Delta u-\nabla \Pi)=\left(a-S_{m} a\right)(\Delta u-\nabla \Pi)+f, \\
\operatorname{div} u=0 \\
\left.u\right|_{t=0}=u_{0}
\end{array}\right.
$$

where $S_{m} a$ is the partial sum of $a$ defined in (2.3). The energy method can then be used to solve (1.7).

To deal with the global wellposedness part of Theorem 1, we observe that as long as $\left\|u_{0}\right\|_{\dot{B}_{2,1}^{1 / 2}}$ is sufficiently small, the lifespan of the local solution thus obtained should be $>1$. Moreover, there exists $t_{1} \in(0,1)$ such that

$$
\left\|u\left(t_{1}\right)\right\|_{\dot{B}_{2,1}^{1 / 2} \cap \dot{B}_{2,1}^{7 / 2}} \leqq C\left\|u_{0}\right\|_{\dot{B}_{2,1}^{1 / 2}} .
$$

We shall first solve $v$ via the classical Navier-Stokes system

$$
\left\{\begin{array}{l}
\partial_{t} v+v \cdot \nabla v-\Delta v+\nabla \Pi_{v}=0, \\
\operatorname{div} v=0 \\
\left.v\right|_{t=t_{1}}=u\left(t_{1}\right),
\end{array}\right.
$$

and then solve $w=u-v$ via

$$
\left\{\begin{array}{l}
\partial_{t} \rho+\operatorname{div}(\rho(v+w))=0, \quad \operatorname{div} w=0 \\
\rho \partial_{t} w+\rho(v+w) \cdot \nabla w-\Delta w+\nabla \Pi_{w}=(1-\rho)\left(\partial_{t} v+v \cdot \nabla v\right)-\rho w \cdot \nabla v \\
\left.\rho\right|_{t=t_{1}}=\rho\left(t_{1}\right),\left.\quad w\right|_{t=t_{1}}=0
\end{array}\right.
$$


This result can be reached through an energy estimate. One may check Remark 10 for the technical reason which precludes using the replacement that $\mathfrak{w} \stackrel{\text { def }}{=} u-v_{L}$ with $v_{L}(t)=\mathrm{e}^{\left(t-t_{1}\right) \Delta} u\left(t_{1}\right)$.

In the second section, we shall collect some basic facts on Littlewood-Paley analysis and various product laws in Besov spaces; then in Section 3 we apply the Littlewood-Paley theory to study the linearized inhomogeneous Navier-Stokes type equations. With these estimates, we shall prove the local-in-time wellposedness part of Theorem 1 in Section 4. Finally in the last section, we present the proof of the global existence part of Theorem 1 .

Let us complete this section by describing the notations we are going to use in this context.

Notations. Let $A, B$ be two operators. We denote $[A, B]=A B-B A$, the commutator between $A$ and $B$. For $a \lesssim b$, we mean that there is a uniform constant $C$, which may be different on different lines, such that $a \leqq C b$. We shall denote by $(a \mid b)\left(\right.$ or $\left.(a \mid b)_{L^{2}}\right)$ the $L^{2}\left(\mathbb{R}^{3}\right)$ inner product of $a$ and $b$.

For $X$ a Banach space and $I$ an interval of $\mathbb{R}$, we denote by $\mathcal{C}(I ; X)$ the set of continuous functions on $I$ with values in $X$, and by $\mathcal{C}_{b}(I ; X)$ the subset of bounded functions of $\mathcal{C}(I ; X)$. For $q \in[1,+\infty]$, the notation $L^{q}(I ; X)$ stands for the set of measurable functions on $I$ with values in $X$, such that $t \longmapsto\|f(t)\|_{X}$ belongs to $L^{q}(I)$.

We always denote the Fourier transform of a function $u$ by $\hat{u}$ or $\mathcal{F}(u)$, by $\left\{c_{j, r}\right\}_{j \in \mathbb{Z}}$ a generic element of the sphere of $\ell^{r}(\mathbb{Z})$ and by $\left(c_{k}\right)_{k \in \mathbb{Z}}\left(\operatorname{resp} .\left(d_{j}\right)_{j \in \mathbb{Z}}\right)$ a generic element of the sphere of $\ell^{2}(\mathbb{Z})\left(\operatorname{resp} . \ell^{1}(\mathbb{Z})\right)$.

\section{The Functional Tool Box}

The proof of Theorem 1 requires a dyadic decomposition of the Fourier variables, or Littlewood-Paley decomposition. Let us briefly explain how this may be built in the case $x \in \mathbb{R}^{3}$ (see for example [4]). Let $\varphi$ be a smooth function supported in the ring $\mathcal{C} \stackrel{\text { def }}{=}\left\{\xi \in \mathbb{R}^{3}, \frac{3}{4} \leqq|\xi| \leqq \frac{8}{3}\right\}$, such that

$$
\sum_{q \in \mathbb{Z}} \varphi\left(2^{-q} \xi\right)=1 \text { for } \xi \neq 0 \text {. }
$$

Then for $u \in \mathcal{S}^{\prime}\left(\mathbb{R}^{3}\right)$, we set

$$
\forall q \in \mathbb{Z}, \quad \dot{\Delta}_{q} u=\varphi\left(2^{-q} \mathrm{D}\right) u \quad \text { and } \quad \dot{S}_{q} u=\sum_{j \leqq q-1} \dot{\Delta}_{j} u .
$$

We have the formal decomposition

$$
u=\sum_{q \in \mathbb{Z}} \dot{\Delta}_{q} u, \quad \forall u \in \mathcal{S}^{\prime}\left(\mathbb{R}^{3}\right) / \mathcal{P}\left[\mathbb{R}^{3}\right]
$$

where $\mathcal{P}\left[\mathbb{R}^{3}\right]$ is the set of polynomials (see [21]). Moreover, the Littlewood-Paley decomposition satisfies the property of almost orthogonality, 


$$
\dot{\Delta}_{k} \dot{\Delta}_{q} u \equiv 0 \quad \text { if }|k-q| \geqq 2 \text { and } \quad \dot{\Delta}_{k}\left(\dot{S}_{q-1} u \dot{\Delta}_{q} u\right) \equiv 0 \quad \text { if }|k-q| \geqq 5 .
$$

We recall now the definition of homogeneous Besov spaces from [22].

Definition 1. Let $(p, r) \in[1,+\infty]^{2}, s \in \mathbb{R}$ and $u \in \mathcal{S}^{\prime}\left(\mathbb{R}^{3}\right)$, we set

$$
\|u\|_{\dot{B}_{p, r}^{s}} \stackrel{\text { def }}{=}\left(2^{q s}\left\|\dot{\Delta}_{q} u\right\|_{L^{p}}\right)_{\ell^{r}} .
$$

- For $s<\frac{3}{p}$ (or $s=\frac{3}{p}$ if $r=1$ ), we define

$$
\dot{B}_{p, r}^{s}\left(\mathbb{R}^{3}\right) \stackrel{\text { def }}{=}\left\{u \in \mathcal{S}^{\prime}\left(\mathbb{R}^{3}\right) \mid\|u\|_{\dot{B}_{p, r}^{s}}<\infty\right\} .
$$

- If $k \in \mathbb{N}$ and $\frac{3}{p}+k \leqq s<\frac{3}{p}+k+1$ (or $s=\frac{3}{p}+k+1$ if $r=1$ ), then $\dot{B}_{p, r}^{s}\left(\mathbb{R}^{3}\right.$ ) is defined as the subset of distributions $u \in \mathcal{S}^{\prime}\left(\mathbb{R}^{3}\right)$ such that $\partial^{\beta} u \in \dot{B}_{p, r}^{s-k}\left(\mathbb{R}^{3}\right)$ whenever $|\beta|=k$.

Remark 1. Similar to Definition 1, we can also define the inhomogeneous Besov spaces. Indeed, let $\chi \in[0,1]$ be a smooth function supported in the ball $\left\{\xi \in \mathbb{R}^{3},|\xi| \leqq \frac{4}{3}\right\}$ such that

$$
\chi(\xi)+\sum_{q \geqq 0} \varphi\left(2^{-q} \xi\right)=1 \text { for } \forall \xi \in \mathbb{R}^{3} .
$$

For $u \in \mathcal{S}^{\prime}\left(\mathbb{R}^{3}\right)$, we set

$$
\begin{aligned}
& \forall q \geqq 0, \quad \Delta_{q} u=\varphi\left(2^{-q} \mathrm{D}\right) u ; \quad \Delta_{-1} u=\chi(\mathrm{D}) u ; \\
& \Delta_{q} u=0 \quad \text { if } \quad q \leqq-2 ; \quad \text { and } \quad S_{q} u=\sum_{j \leqq q-1} \Delta_{j} u .
\end{aligned}
$$

Then for all $u \in \mathcal{S}^{\prime}\left(\mathbb{R}^{3}\right)$, we have the inhomogeneous Littlewood-Paley decomposition $u=\sum_{q \in \mathbb{Z}} \Delta_{q} u$, and for $(p, r) \in[1,+\infty]^{2}, s \in \mathbb{R}$ and $u \in \mathcal{S}^{\prime}\left(\mathbb{R}^{3}\right)$, we define the inhomogeneous Besov space $B_{p, r}^{s}\left(\mathbb{R}^{3}\right)$ as

$$
B_{p, r}^{s}\left(\mathbb{R}^{3}\right) \stackrel{\text { def }}{=}\left\{u \in \mathcal{S}^{\prime}\left(\mathbb{R}^{3}\right) \mid\|u\|_{B_{p, r}^{s}} \stackrel{\text { def }}{=}\left(2^{q s}\left\|\Delta_{q} u\right\|_{L^{p}}\right)_{\ell^{r}}<\infty\right\} .
$$

Remark 2. 1. We point out that if $s>0$ then $B_{p, r}^{s}\left(\mathbb{R}^{3}\right)=\dot{B}_{p, r}^{s}\left(\mathbb{R}^{3}\right) \cap L^{p}\left(\mathbb{R}^{3}\right)$ and

$$
\|u\|_{B_{p, r}^{s}} \approx\|u\|_{\dot{B}_{p, r}^{s}}+\|u\|_{L^{p}} .
$$

2. Let $s \in \mathbb{R}, 1 \leqq p, r \leqq \infty$, and $u \in \mathcal{S}^{\prime}\left(\mathbb{R}^{3}\right)$. Then $u$ belongs to $\dot{B}_{p, r}^{s}\left(\mathbb{R}^{3}\right)$ if and only if there exists $\left\{c_{j, r}\right\}_{j \in \mathbb{Z}}$ such that $\left\|c_{j, r}\right\|_{\ell^{r}}=1$ and

$$
\left\|\dot{\Delta}_{j} u\right\|_{L^{p}} \leqq C c_{j, r} 2^{-j s}\|u\|_{\dot{B}_{p, r}^{s}} \quad \text { for all } j \in \mathbb{Z} .
$$

For the convenience of the reader, we recall some basic facts of Littlewood-Paley theory; one may refer to $[4,5,22]$ for more details. 
Lemma 1. Let $\mathcal{B}$ be a ball and $\mathcal{C}$ a ring of $\mathbb{R}^{3}$. A constant $C$ exists so that for any positive real number $\lambda$, any non-negative integer $k$, any homogeneous function $\sigma$ of degree $m$ smooth outside of 0 , and any pair of real numbers $(a, b)$ with $b \geqq a \geqq 1$, the following hold:

$$
\begin{aligned}
& \text { Supp } \hat{u} \subset \lambda \mathcal{B} \Rightarrow \sup _{|\alpha|=k}\left\|\partial^{\alpha} u\right\|_{L^{b}} \leqq C^{k+1} \lambda^{k+3\left(\frac{1}{a}-\frac{1}{b}\right)}\|u\|_{L^{a}}, \\
& \text { Supp } \hat{u} \subset \lambda \mathcal{C} \Rightarrow C^{-1-k} \lambda^{k}\|u\|_{L^{a}} \leqq \sup _{|\alpha|=k}\left\|\partial^{\alpha} u\right\|_{L^{a}} \leqq C^{1+k} \lambda^{k}\|u\|_{L^{a}}, \\
& \text { Supp } \hat{u} \subset \lambda \mathcal{C} \Rightarrow\|\sigma(D) u\|_{L^{b}} \leqq C_{\sigma, m} \lambda^{m+3\left(\frac{1}{a}-\frac{1}{b}\right)}\|u\|_{L^{a}} .
\end{aligned}
$$

In what follows, we shall frequently use Bony's decomposition [5] in both the homogeneous and the inhomogeneous context:

$$
\begin{aligned}
& u v=\dot{T}_{u} v+\dot{R}(u, v)=\dot{T}_{u} v+\dot{T}_{v} u+\dot{\mathcal{R}}(u, v) \text { and } \\
& u v=T_{u} v+R(u, v)=T_{u} v+T_{v} u+\mathcal{R}(u, v)
\end{aligned}
$$

where

$$
\begin{aligned}
& \dot{T}_{u} v \stackrel{\text { def }}{=} \sum_{q \in \mathbb{Z}} \dot{S}_{q-1} u \dot{\Delta}_{q} v, \quad \dot{R}(u, v) \stackrel{\text { def }}{=} \sum_{q \in \mathbb{Z}} \dot{\Delta}_{q} u \dot{S}_{q+2} v, \\
& \dot{\mathcal{R}}(u, v) \stackrel{\text { def }}{=} \sum_{q \in \mathbb{Z}} \dot{\Delta}_{q} u \widetilde{\dot{\Delta}}_{q} v \text { and } \widetilde{\dot{\Delta}}_{q} v \stackrel{\text { def }}{=} \sum_{\left|q^{\prime}-q\right| \leqq 1} \dot{\Delta}_{q^{\prime}} v,
\end{aligned}
$$

and similar definitions for $T_{u} v, R(u, v)$ and $\mathcal{R}(u, v)$.

In order to obtain a better description of the regularizing effect of the transportdiffusion equation, we will use Chemin-Lerner type spaces $\widetilde{L}_{T}^{\lambda}\left(\dot{B}_{p, r}^{s}\left(\mathbb{R}^{3}\right)\right)$ from $[6,8]$.

Definition 2. Let $s \leqq \frac{3}{p}$ (resp. $\left.s \in \mathbb{R}\right),(r, \lambda, p) \in[1,+\infty]^{3}$ and $\left.\left.T \in\right] 0,+\infty\right]$. We define $\widetilde{L}_{T}^{\lambda}\left(\dot{B}_{p r}^{s}\left(\mathbb{R}^{3}\right)\right)$ as the completion of $\mathcal{C}\left([0, T] ; \mathcal{S}\left(\mathbb{R}^{3}\right)\right)$ by the norm

$$
\|f\|_{\widetilde{L}_{T}^{\lambda}\left(\dot{B}_{p, r}^{s}\right)} \stackrel{\operatorname{def}}{=}\left(\sum_{q \in \mathbb{Z}} 2^{q r s}\left(\int_{0}^{T}\left\|\dot{\Delta}_{q} f(t)\right\|_{L^{p}}^{\lambda} \mathrm{d} t\right)^{\frac{r}{\lambda}}\right)^{\frac{1}{r}}<\infty
$$

with the usual change if $r=\infty$. For short, we denote this space by $\widetilde{L}_{T}^{\lambda}\left(\dot{B}_{p, r}^{s}\right)$. In the particular case when $p=r=2$, we denote this space by $\widetilde{L}_{T}^{\lambda}\left(\dot{H}^{s}\right)$.

\section{Preliminaries}

For the sake of completeness, we shall first recall the following commutator's estimates which will be frequently used throughout the succeeding sections.

Lemma 2. [2] Let $r \in[1, \infty], u \in \dot{B}_{2, r}^{s}\left(\mathbb{R}^{3}\right)$ and $v \in \dot{B}_{2,1}^{5 / 2}\left(\mathbb{R}^{3}\right)$ with $\operatorname{div} v=0$. Then there hold 
(i) if $-\frac{5}{2}<s<\frac{5}{2}$ (or $s=\frac{5}{2}$ with $\left.r=1\right)$,

$$
\left\|\left[\dot{\Delta}_{q}, v \cdot \nabla\right] u\right\|_{L^{2}} \lesssim c_{q, r} 2^{-s q}\|v\|_{\dot{B}_{2,1}^{5 / 2}}\|u\|_{\dot{B}_{2, r}^{s}} .
$$

(ii) If $s>-1, u=v$,

$$
\left\|\left[\dot{\Delta}_{q}, v \cdot \nabla\right] v\right\|_{L^{2}} \lesssim d_{q} 2^{-s q}\|\nabla v\|_{L^{\infty}}\|v\|_{\dot{B}_{2,1}^{s}} .
$$

Remark 3. In the framework of inhomogeneous Besov spaces, thanks to (3.1), (2.4) and Lemma 2.2 in [10], we have for $0<s<5 / 2$ (or $s=5 / 2$ with $r=1$ )

$$
\left\|\left[\Delta_{q}, v \cdot \nabla\right] u\right\|_{L^{2}} \lesssim c_{q, r} 2^{-s q}\|v\|_{\dot{B}_{2,1}^{5 / 2}}\|u\|_{B_{2, r}^{s}}
$$

and

$$
\left\|\left[\Delta_{q}, v \cdot \nabla\right] u\right\|_{L^{2}} \lesssim d_{q} 2^{-s q}\|\nabla v\|_{B_{2,1}^{3 / 2}}\|u\|_{B_{2,1}^{s}} \quad \text { with }-5 / 2<s \leqq 5 / 2 .
$$

Thanks to Lemma 2, we have

Proposition 1. [2] Let $v$ be a divergence free vector field with $\nabla v \in L^{1}([0, T]$; $\left.\dot{B}_{2,1}^{3 / 2}\left(\mathbb{R}^{3}\right)\right)$. For $s \in\left(-\frac{5}{2}, \frac{5}{2}\right]$, given $f_{0} \in \dot{B}_{2,1}^{s}\left(\mathbb{R}^{3}\right), F \in L^{1}\left([0, T] ; \dot{B}_{2,1}^{s}\left(\mathbb{R}^{3}\right)\right)$, the following transport equation

$$
\left\{\begin{array}{l}
\partial_{t} f+v \cdot \nabla f=F \text { in } \mathbb{R}^{+} \times \mathbb{R}^{3} \\
\left.f\right|_{t=0}=f_{0}
\end{array}\right.
$$

has a unique solution $f \in \mathcal{C}\left([0, T] ; \dot{B}_{2,1}^{s}\left(\mathbb{R}^{3}\right)\right)$. Moreover, there holds for all $t \in[0, T]$

$$
\|f\|_{\widetilde{L}_{t}^{\infty}\left(\dot{B}_{2,1}^{s}\right)} \leqq\left\|f_{0}\right\|_{\dot{B}_{2,1}^{s}}+C \int_{0}^{t}\left\|f\left(t^{\prime}\right)\right\|_{\dot{B}_{2,1}^{s}}\left\|\nabla v\left(t^{\prime}\right)\right\|_{\dot{B}_{2,1}^{3 / 2}} d t^{\prime}+C\|F\|_{L_{t}^{1}\left(\dot{B}_{2,1}^{s}\right)}
$$

Remark 4. [2] In the framework of inhomogeneous Besov spaces, there similarly holds for $s \in\left(0, \frac{5}{2}\right]$

$$
\|f\|_{\widetilde{L}_{t}^{\infty}\left(B_{2,1}^{s}\right)} \leqq\left\|f_{0}\right\|_{B_{2,1}^{s}}+C \int_{0}^{t}\left\|f\left(t^{\prime}\right)\right\|_{B_{2,1}^{s}}\left\|\nabla v\left(t^{\prime}\right)\right\|_{\dot{B}_{2,1}^{3 / 2}} d t^{\prime}+C\|F\|_{L_{t}^{1}\left(B_{2,1}^{s}\right)} .
$$

Proposition 2. Let $m \in \mathbb{Z}, a_{0} \in \dot{B}_{2,1}^{3 / 2}\left(\mathbb{R}^{3}\right), u \in L_{T}^{1}\left(\dot{B}_{2,1}^{5 / 2}\right)$ with $\operatorname{div} u=0$, and $\left.a \in \mathcal{C}\left([0, T] ; \dot{B}_{2,1}^{3 / 2}\left(\mathbb{R}^{3}\right)\right)\right)$ such that $(a, u)$ solves

$$
\left\{\begin{array}{l}
\partial_{t} a+u \cdot \nabla a=0 \\
\left.a\right|_{t=0}=a_{0}
\end{array}\right.
$$

Then there holds for $\forall t \leqq T$

$$
\left\|a-\dot{S}_{m} a\right\|_{\widetilde{L}_{t}^{\infty}\left(\dot{B}_{2,1}^{3 / 2}\right)} \leqq \sum_{q \geqq m} 2^{3 q / 2}\left\|\dot{\Delta}_{q} a_{0}\right\|_{L^{2}}+\left\|a_{0}\right\|_{\dot{B}_{2,1}^{3 / 2}}\left(e^{C U(t)}-1\right),
$$

with $U(t)=\|u\|_{L_{t}^{1}\left(\dot{B}_{2,1}^{5 / 2}\right)}$. 
Proof. Applying $\dot{\Delta}_{q}$ to (3.8) yields

$$
\partial_{t} \dot{\Delta}_{q} a+u \cdot \nabla \dot{\Delta}_{q} a=\left[u \cdot \nabla, \dot{\Delta}_{q}\right] a .
$$

Taking the $L^{2}$ inner product of (3.10) with $\dot{\Delta}_{q} a$, and then performing a time integration, we obtain

$$
\left\|\dot{\Delta}_{q} a(t)\right\|_{L^{2}} \leqq\left\|\dot{\Delta}_{q} a_{0}\right\|_{L^{2}}+\int_{0}^{t}\left\|\left[u \cdot \nabla, \dot{\Delta}_{q}\right] a\right\|_{L^{2}}(\tau) \mathrm{d} \tau,
$$

while, thanks to (3.1), we have

$$
\left\|\left[u \cdot \nabla, \dot{\Delta}_{q}\right] a\right\|_{L^{2}} \lesssim d_{q} 2^{-3 q / 2}\|u\|_{\dot{B}_{2,1}^{5 / 2}}\|a\|_{\dot{B}_{2,1}^{3 / 2}}
$$

which together with the fact deduced from Proposition 1 that $\|a(t)\|_{\dot{B}_{2,1}^{3 / 2}}$ $\leqq C\left\|a_{0}\right\|_{\dot{B}_{2,1}^{3 / 2}} \mathrm{e}^{C U(t)}$, implies

$2^{3 q / 2}\left\|\dot{\Delta}_{q} a(t)\right\|_{L_{t}^{\infty}\left(L^{2}\right)} \leqq 2^{3 q / 2}\left\|\dot{\Delta}_{q} a_{0}\right\|_{L^{2}}+\left\|a_{0}\right\|_{\dot{B}_{2,1}^{3 / 2}} \int_{0}^{t} d_{q}(\tau) C U^{\prime}(\tau) \mathrm{e}^{C U(\tau)} \mathrm{d} \tau$.

Then, summing up (3.11) on $\{q \geqq m\}$ leads to (3.9).

Remark 5. In the framework of inhomogeneous Besov spaces, it follows from the same line of the proof to (3.9) that $\forall t \leqq T$

$$
\left\|a-S_{m} a\right\|_{\widetilde{L}_{t}^{\infty}\left(B_{2,1}^{3 / 2}\right)} \leqq \sum_{q \geqq m} 2^{3 q / 2}\left\|\Delta_{q} a_{0}\right\|_{L^{2}}+\left\|a_{0}\right\|_{B_{2,1}^{3 / 2}}\left(\mathrm{e}^{C U(t)}-1\right)
$$

with $U(t)=\|u\|_{L_{t}^{1}\left(\dot{B}_{2,1}^{5 / 2}\right)}$.

To deal with the pressure term in (1.2), we need:

Lemma 3. (i) Let $a \in B_{2,1}^{5 / 2}\left(\mathbb{R}^{3}\right)$ and $\nabla \Pi \in L^{2}\left(\mathbb{R}^{3}\right)$, then there holds

$$
\left\|\left[\Delta_{q}, a\right] \nabla \Pi\right\|_{L^{2}} \lesssim c_{q} 2^{-q}\|a\|_{B_{2,1}^{5 / 2}}\|\nabla \Pi\|_{L^{2}}
$$

(ii) Let $a \in H^{2}\left(\mathbb{R}^{3}\right)$ and $\nabla \Pi \in H^{-1}\left(\mathbb{R}^{3}\right)$, then there holds

$$
\left\|\left[\Delta_{q}, a\right] \nabla \Pi\right\|_{L^{2}} \lesssim d_{q} 2^{q / 2}\|a\|_{H^{2}}\|\nabla \Pi\|_{H^{-1}} .
$$

Proof. Again thanks to (2.6), we get by a standard commutator argument that

$$
\left[\Delta_{q}, a\right] \nabla \Pi=\left[\Delta_{q}, T_{a}\right] \nabla \Pi+\Delta_{q} T_{\nabla \Pi} a+\Delta_{q} \mathcal{R}(a, \nabla \Pi)-R\left(a, \Delta_{q} \nabla \Pi\right) .
$$

We first deduce from $\left\|S_{k-1} \nabla a\right\|_{L^{\infty}} \lesssim\|\nabla a\|_{B_{2,1}^{3 / 2}}$ that

$$
\left\|\left[\Delta_{q}, T_{a}\right] \nabla \Pi\right\|_{L^{2}} \lesssim 2^{-q} \sum_{|k-q| \leqq 4}\left\|S_{k-1} \nabla a\right\|_{L^{\infty}}\left\|\nabla \Delta_{k} \Pi\right\|_{L^{2}} \lesssim c_{q} 2^{-q}\|a\|_{B_{2,1}^{5 / 2}}\|\nabla \Pi\|_{L^{2}}
$$


and as $\left\|S_{k-1} \nabla \Pi\right\|_{L^{\infty}} \lesssim c_{k} 2^{3 k / 2}\|\nabla \Pi\|_{L^{2}}$, which leads to

$$
\left\|\Delta_{q} T_{\nabla \Pi a} a\right\|_{L^{2}} \lesssim \sum_{|q-k| \leqq 4}\left\|S_{k-1} \nabla \Pi\right\|_{L^{\infty}}\left\|\Delta_{k} a\right\|_{L^{2}} \lesssim d_{q} 2^{-q}\|a\|_{H^{5 / 2}}\|\nabla \Pi\|_{L^{2}}
$$

Applying Lemma 1 again gives

$$
\begin{aligned}
& \left\|\Delta_{q} \mathcal{R}(a, \nabla \Pi)\right\|_{L^{2}} \lesssim 2^{3 q / 2} \sum_{k \geqq q-3}\left\|\Delta_{k} a\right\|_{L^{2}}\left\|\widetilde{\Delta}_{k} \nabla \Pi\right\|_{L^{2}} \\
& \lesssim 2^{3 q / 2}\left(\sum_{k \geqq q-3} c_{k}^{2} 2^{-5 k / 2}\right)\|a\|_{H^{5 / 2}}\|\nabla \Pi\|_{L^{2}} \lesssim d_{q} 2^{-q}\|a\|_{H^{5 / 2}}\|\nabla \Pi\|_{L^{2}},
\end{aligned}
$$

and a similar argument gives the same estimate for $\left\|R\left(a, \Delta_{q} \nabla \Pi\right)\right\|_{L^{2}}$. Whence, thanks to (3.15), we arrive at (3.13).

Finally, let us turn to the proof of (3.14). We get first from $\left\|S_{k-1} \nabla a\right\|_{L^{\infty}}$ $\lesssim c_{k} 2^{k / 2}\|a\|_{H^{2}}$ that

$$
\begin{aligned}
\left\|\left[\Delta_{q}, T_{a}\right] \nabla \Pi\right\|_{L^{2}} & \lesssim 2^{-q} \sum_{|k-q| \leqq 4}\left\|S_{k-1} \nabla a\right\|_{L^{\infty}}\left\|\nabla \Delta_{k} \Pi\right\|_{L^{2}} \\
& \lesssim d_{q} 2^{q / 2}\|a\|_{H^{2}}\|\nabla \Pi\|_{H^{-1}} .
\end{aligned}
$$

Note that $\left\|S_{k-1} \nabla \Pi\right\|_{L^{\infty}} \lesssim c_{k} 2^{5 k / 2}\|\nabla \Pi\|_{H^{-1}}$, which leads to

$$
\begin{aligned}
\left\|\Delta_{q} T_{\nabla \Pi} a\right\|_{L^{2}} & \lesssim \sum_{|q-k| \leqq 4}\left\|S_{k-1} \nabla \Pi\right\|_{L^{\infty}}\left\|\Delta_{k} a\right\|_{L^{2}} \\
& \lesssim d_{q} 2^{q / 2}\|a\|_{H^{2}}\|\nabla \Pi\|_{H^{-1}} .
\end{aligned}
$$

Applying Lemma 1 once again yields

$$
\begin{aligned}
& \left\|\Delta_{q} \mathcal{R}(a, \nabla \Pi)\right\|_{L^{2}} \lesssim 2^{3 q / 2} \sum_{k \geqq q-3}\left\|\Delta_{k} a\right\|_{L^{2}}\left\|\widetilde{\Delta}_{k} \nabla \Pi\right\|_{L^{2}} \\
& \lesssim 2^{3 q / 2}\left(\sum_{k \geqq q-3} c_{k}^{2} 2^{-k}\right)\|a\|_{H^{2}}\|\nabla \Pi\|_{H^{-1}} \lesssim d_{q} 2^{q / 2}\|a\|_{H^{2}}\|\nabla \Pi\|_{H^{-1}} .
\end{aligned}
$$

The same estimate holds for $\left\|R\left(a, \Delta_{q} \nabla \Pi\right)\right\|_{L^{2}}$. Combining the above estimates with (3.15), we conclude the proof of (3.14).

Proposition 3. Let $\boldsymbol{F}=\left(F_{1}, F_{2}, F_{3}\right) \in L_{T}^{1}\left(B_{2,1}^{-1 / 2}\right), a \in L_{T}^{\infty}\left(H^{2}\right)$ with $\underline{b} \stackrel{\text { def }}{=}$ $\inf _{(t, x) \in[0, T] \times \mathbb{R}^{3}}(1+a(t, x))>0$ and $\nabla \Pi \in L_{T}^{1}\left(\dot{H}^{-1}\right)$, which solves

(E) $\operatorname{div}((1+a) \nabla \Pi)=\operatorname{div} \boldsymbol{F}$.

Then there holds

$$
\underline{b}\|\nabla \Pi\|_{L_{T}^{1}\left(B_{2,1}^{-1 / 2}\right)} \leqq C\left(\|\boldsymbol{F}\|_{L_{T}^{1}\left(B_{2,1}^{-1 / 2}\right)}+\|a\|_{L_{T}^{\infty}\left(H^{2}\right)}\|\nabla \Pi\|_{L_{T}^{1}\left(H^{-1}\right)}\right) .
$$


Proof. Acting $\dot{\Delta}_{q}$ to $(E)$ and taking the $L^{2}$ inner product of the resulting equation with $\dot{\Delta}_{q} \Pi$, we get

$$
\left((1+a) \dot{\Delta}_{q} \nabla \Pi \mid \dot{\Delta}_{q} \nabla \Pi\right)_{L^{2}}=\left(\dot{\Delta}_{q} \boldsymbol{F} \mid \dot{\Delta}_{q} \nabla \Pi\right)_{L^{2}}+\left(\left[a, \dot{\Delta}_{q}\right] \nabla \Pi \mid \dot{\Delta}_{q} \nabla \Pi\right)_{L^{2}} .
$$

Taking square root of the above equality and then integrating the resulting inequality over $[0, T]$ gives

$$
\underline{b}\left\|\dot{\Delta}_{q} \nabla \Pi\right\|_{L_{T}^{1}\left(L^{2}\right)} \leqq\left\|\dot{\Delta}_{q} \boldsymbol{F}\right\|_{L_{T}^{1}\left(L^{2}\right)}+\int_{0}^{T}\left\|\left[a, \dot{\Delta}_{q}\right] \nabla \Pi\left(t^{\prime}\right)\right\|_{L^{2}} \mathrm{~d} t^{\prime} .
$$

Then, applying (3.14)-(3.19) yields (3.18).

Proposition 4. Let $a \in B_{2,1}^{3 / 2}\left(\mathbb{R}^{3}\right)$ such that $0<\underline{b} \leqq 1+a \leqq \bar{b}$, and

$$
\left\|a-S_{m} a\right\|_{B_{2,1}^{3 / 2}} \leqq c
$$

for some sufficiently small positive constant $c$ and some integer $m \in \mathbb{Z}$. Let $F \in H^{-1}\left(\mathbb{R}^{3}\right)$ and $\nabla \Pi \stackrel{\text { def }}{=} \mathcal{H}_{b}(F) \in H^{-1}\left(\mathbb{R}^{3}\right)$ solves

$$
\operatorname{div}((1+a) \nabla \Pi)=\operatorname{div} F .
$$

Then there holds

$$
\|\nabla \Pi\|_{H^{-1}} \lesssim\left(1+2^{m}\|a\|_{B_{2,1}^{3 / 2}}\right)\|F\|_{H^{-1}}
$$

Proof. We first deduce from (3.20) and $\underline{b} \leqq 1+a$ that

$$
1+S_{m} a=1+a+\left(S_{m} a-a\right) \geqq \frac{b}{2} .
$$

Motivated by [12], we shall use a duality argument to prove (3.22). For the sake of simplicity, we just prove (3.22) for sufficiently smooth function $F$. In order to make the following computation rigorous, one has to use a density argument, which we omit here.

For this, we first estimate $\|\nabla \Pi\|_{H^{1}}$ under the assumption that $F \in H^{1}\left(\mathbb{R}^{3}\right)$. Indeed, we write

$$
\operatorname{div}\left[\left(1+S_{m} a\right) \nabla \Pi\right]=\operatorname{div} F+\operatorname{div}\left[\left(S_{m} a-a\right) \nabla \Pi\right]
$$

applying $\Delta_{q}$ to the above equation gives

$\operatorname{div}\left[\left(1+S_{m} a\right) \Delta_{q} \nabla \Pi\right]=\operatorname{div} \Delta_{q} F+\operatorname{div} \Delta_{q}\left[\left(S_{m} a-a\right) \nabla \Pi\right]+\operatorname{div}\left(\left[S_{m} a, \Delta_{q}\right] \nabla \Pi\right)$.

Taking the $L^{2}$ inner product of this equation with $\Delta_{q} \Pi$ and using (3.13), we obtain

$$
\begin{aligned}
\|\nabla \Pi\|_{H^{1}} & \lesssim\left\|\left(S_{m} a-a\right) \nabla \Pi\right\|_{H^{1}}+\|F\|_{H^{1}}+\left\|S_{m} a\right\|_{B_{2,1}^{5 / 2}\|\nabla \Pi\|_{L^{2}}} \\
& \lesssim\left\|S_{m} a-a\right\|_{B_{2,1}^{3 / 2}}\|\nabla \Pi\|_{H^{1}}+\left(1+2^{m}\|a\|_{\left.B_{2,1}^{3 / 2}\right)\|F\|_{H^{1}}}\right.
\end{aligned}
$$


where we used the classical elliptic estimate $\|\nabla \Pi\|_{L^{2}} \lesssim\|F\|_{L^{2}}$. This along with (3.20) leads to

$$
\|\nabla \Pi\|_{H^{1}} \lesssim\left(1+2^{m}\|a\|_{B_{2,1}^{3 / 2}}\right)\|F\|_{H^{1}} .
$$

Now we use a duality argument to estimate $\|\nabla \Pi\|_{H^{-1}}$ in the case when $F \in H^{-1}\left(\mathbb{R}^{3}\right)$. Notice that

$$
\|\nabla \Pi\|_{H^{-1}}=\sup _{\|g\|_{H^{1}} \leqq 1}\langle g, \nabla \Pi\rangle=\sup _{\|g\|_{H^{1}} \leqq 1}-\int \Pi \operatorname{div} g \mathrm{~d} x,
$$

where $\langle g, \nabla \Pi\rangle$ denotes the duality bracket between $\mathcal{S}^{\prime}\left(\mathbb{R}^{3}\right)$ and $\mathcal{S}\left(\mathbb{R}^{3}\right)$, whereas (3.24) ensures that for any $g \in H^{1}\left(\mathbb{R}^{3}\right)$

$$
\operatorname{div}\left((1+a) \nabla h_{g}\right)=\operatorname{div} g
$$

has a unique solution $\nabla h_{g} \in H^{1}\left(\mathbb{R}^{3}\right)$ satisfying

$$
\left\|\nabla h_{g}\right\|_{H^{1}} \lesssim\left(1+2^{m}\|a\|_{B_{2,1}^{3 / 2}}\right)\|g\|_{H^{1}},
$$

which along with (3.25) yields

$$
\begin{aligned}
& \|\nabla \Pi\|_{H^{-1}}=\sup _{\|g\|_{H^{1}} \leqq 1}-\left\langle\Pi, \operatorname{div}\left((1+a) \nabla h_{g}\right)\right\rangle \\
& =\sup _{\|g\|_{H^{1}} \leqq 1}\left\langle(1+a) \nabla \Pi, \nabla h_{g}\right\rangle=\sup _{\|g\|_{H^{1}} \leqq 1}-\left\langle h_{g}, \operatorname{div}((1+a) \nabla \Pi)\right\rangle \\
& =\sup _{\|g\|_{H^{1}} \leqq 1}-\left\langle\operatorname{div} F, h_{g}\right\rangle=\sup _{\|g\|_{H^{1}} \leqq 1}\left\langle F, \nabla h_{g}\right\rangle .
\end{aligned}
$$

Thanks to (3.26), we obtain

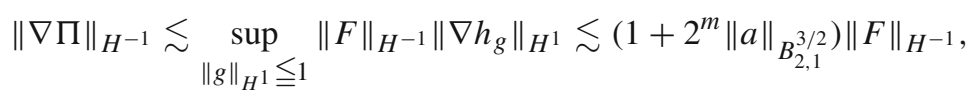

which completes the proof of this proposition.

Proposition 5. Let $u_{0} \in B_{2,1}^{-1 / 2}\left(\mathbb{R}^{3}\right)$ and $v$ be a divergence free vector field satisfying $\nabla v \in L_{T}^{1}\left(B_{2,1}^{3 / 2}\right)$. Let $f \in L_{T}^{1}\left(B_{2,1}^{-1 / 2}\right)$ and $a \in L_{T}^{\infty}\left(H^{2}\right)$ with $1+a \geqq c_{1}>0$. We assume that $u \in L_{T}^{\infty}\left(B_{2,1}^{-1 / 2}\right) \cap L_{T}^{1}\left(B_{2,1}^{3 / 2}\right)$ and $\nabla \Pi \in L_{T}^{1}\left(H^{-1}\right)$, which solves

$$
\left\{\begin{array}{l}
\partial_{t} u+v \cdot \nabla u-(1+a)(\Delta u-\nabla \Pi)=f, \quad(t, x) \in \mathbb{R}^{+} \times \mathbb{R}^{3}, \\
\operatorname{div} u=0 \\
u_{\mid t=0}=u_{0} .
\end{array}\right.
$$

Then there holds:

$$
\begin{aligned}
& \|u\|_{\widetilde{L}_{T}^{\infty}\left(B_{2,1}^{-1 / 2}\right)}+\|u\|_{L_{T}^{1}\left(B_{2,1}^{3 / 2}\right)} \\
& \leq C\left\{\left\|u_{0}\right\|_{B_{2,1}^{-1 / 2}}+\|f\|_{L_{T}^{1}\left(B_{2,1}^{-1 / 2}\right)}+\int_{0}^{T}\|u(t)\|_{B_{2,1}^{-1 / 2}}\|\nabla v(t)\|_{B_{2,1}^{3 / 2}} d t\right. \\
& \left.\quad+\|a\|_{L_{T}^{\infty}\left(H^{2}\right)}\left(\|\nabla \Pi\|_{L_{T}^{1}\left(H^{-1}\right)}+\|\nabla u\|_{L_{T}^{1}\left(L^{2}\right)}\right)+\|u\|_{L_{T}^{1}\left(L^{2}\right)}\right\}
\end{aligned}
$$


Proof. Applying $\Delta_{q}$ to (3.28), then a standard commutator process gives

$$
\begin{aligned}
\partial_{t} & \Delta_{q} u+(v \cdot \nabla) \Delta_{q} u-\operatorname{div}\left((1+a) \nabla \Delta_{q} u\right)+\Delta_{q} \nabla\left(T_{1+a} \Pi\right) \\
= & {\left[v, \Delta_{q}\right] \cdot \nabla u-\Delta_{q}(\nabla a \cdot \nabla u)+\Delta_{q}\left(T_{\nabla a} \Pi\right)-\Delta_{q} T_{\nabla \Pi} a } \\
& -\Delta_{q} \mathcal{R}(\nabla \Pi, a)+\operatorname{div}\left[\Delta_{q}, a\right] \nabla u+\Delta_{q} f .
\end{aligned}
$$

Denoting $u^{H} \stackrel{\text { def }}{=} u-\Delta_{-1} u$, and thanks to the fact that $\operatorname{div} u=\operatorname{div} v=0$ and $1+a \geqq c_{1}$, we get by taking the $L^{2}$ inner product of (3.30) with $\Delta_{q} u$,

$$
\begin{aligned}
& \frac{\mathrm{d}}{\mathrm{d} t}\left\|\Delta_{q} u\right\|_{L^{2}}^{2}+2^{2 q}\left\|\Delta_{q} u^{H}\right\|_{L^{2}}^{2} \\
& \quad \lesssim\left\|\Delta_{q} u\right\|_{L^{2}}\left(\left\|\left[v, \Delta_{q}\right] \cdot \nabla u\right\|_{L^{2}}+\left\|\Delta_{q}(\nabla a \cdot \nabla u)\right\|_{L^{2}}+2^{q}\left\|\left[\Delta_{q}, a\right] \nabla u\right\|_{L^{2}}\right. \\
& \left.\quad+\left\|\Delta_{q}\left(T_{\nabla a} \Pi\right)\right\|_{L^{2}}+\left\|\Delta_{q} T_{\nabla \Pi a} a\right\|_{L^{2}}+\left\|\Delta_{q} \mathcal{R}(\nabla \Pi, a)\right\|_{L^{2}}+\left\|\Delta_{q} f\right\|_{L^{2}}\right) .
\end{aligned}
$$

This leads to

$$
\begin{aligned}
& \left\|\Delta_{q} u\right\|_{L_{t}^{\infty}\left(L^{2}\right)}+2^{2 q}\left\|\Delta_{q} u^{H}\right\|_{L_{t}^{1}\left(L^{2}\right)} \\
& \lesssim\left\|\Delta_{q} u_{0}\right\|_{L^{2}}+\int_{0}^{t}\left(\left\|\left[v, \Delta_{q}\right] \cdot \nabla u\right\|_{L^{2}}+\left\|\Delta_{q}(\nabla a \cdot \nabla u)\right\|_{L^{2}}\right. \\
& \quad+2^{q}\left\|\left[\Delta_{q}, a\right] \nabla u\right\|_{L^{2}}+\left\|\Delta_{q}\left(T_{\nabla a} \Pi\right)\right\|_{L^{2}} \\
& \left.\quad+\left\|\Delta_{q} T_{\nabla \Pi} a\right\|_{L^{2}}+\left\|\Delta_{q} \mathcal{R}(\nabla \Pi, a)\right\|_{L^{2}}+\left\|\Delta_{q} f\right\|_{L^{2}}\right) \mathrm{d} t^{\prime} .
\end{aligned}
$$

Thanks to (2.6), we write

$$
\left[\Delta_{q}, a\right] \nabla u=\Delta_{q} \mathcal{R}(a, \nabla u)+\Delta_{q} T_{\nabla u} a-R\left(a, \Delta_{q} \nabla u\right)-\left[T_{a}, \Delta_{q}\right] \nabla u .
$$

Whereas, applying Lemma 1 gives

$$
\begin{aligned}
\left\|\Delta_{q} \mathcal{R}(a, \nabla u)(t)\right\|_{L^{2}} & \lesssim 2^{3 q / 2} \sum_{k \geqq q-3}\left\|\Delta_{k} a(t)\right\|_{L^{2}}\left\|\widetilde{\Delta}_{k} \nabla u(t)\right\|_{L^{2}} \\
& \lesssim 2^{3 q / 2} \sum_{k \geqq q-3} c_{k}^{2}(t) 2^{-2 k}\|a(t)\|_{H^{2}}\|\nabla u(t)\|_{L^{2}} \\
& \lesssim d_{q}(t) 2^{-q / 2}\|a(t)\|_{H^{2}}\|\nabla u(t)\|_{L^{2}} .
\end{aligned}
$$

The same estimate holds for $\left\|R\left(a, \Delta_{q} \nabla u\right)(t)\right\|_{L^{2}}$. Note that

$$
\left\|S_{k-1} \nabla u(t)\right\|_{L^{\infty}} \lesssim c_{k}(t) 2^{3 k / 2}\|\nabla u(t)\|_{L^{2}}
$$

This along with Lemma 1 leads to

$$
\begin{aligned}
\left\|\Delta_{q} T_{\nabla u} a(t)\right\|_{L^{2}} & \lesssim \sum_{|q-k| \leqq 4}\left\|\Delta_{k} a(t)\right\|_{L^{2}}\left\|S_{k-1} \nabla u(t)\right\|_{L^{\infty}} \\
& \lesssim \sum_{|q-k| \leqq 4} c_{k}^{2}(t) 2^{-k / 2}\|a(t)\|_{H^{2}}\|\nabla u(t)\|_{L^{2}} \\
& \lesssim d_{q}(t) 2^{-q / 2}\|a(t)\|_{H^{2}}\|\nabla u(t)\|_{L^{2}}
\end{aligned}
$$


Finally, notice that $\left\|\nabla S_{k-1} a(t)\right\|_{L^{\infty}} \lesssim c_{k}(t) 2^{k / 2}\|a(t)\|_{H^{2}}$, one has

$$
\begin{aligned}
\left\|\left[T_{a}, \Delta_{q}\right] \nabla u(t)\right\|_{L^{2}} & \lesssim \sum_{|k-q| \leqq 4} 2^{-q}\left\|\nabla S_{k-1} a(t)\right\|_{L^{\infty}}\left\|\Delta_{k} \nabla u(t)\right\|_{L^{2}} \\
& \lesssim \sum_{|k-q| \leqq 4} c_{k}^{2}(t) 2^{-k}\|\nabla u(t)\|_{L^{2}} 2^{k / 2}\|a(t)\|_{H^{2}} \\
& \lesssim d_{q}(t) 2^{-q / 2}\|\nabla u(t)\|_{L^{2}}\|a(t)\|_{H^{2}} .
\end{aligned}
$$

As a consequence, we obtain

$$
\left\|\left[\Delta_{q}, a\right] \nabla u(t)\right\|_{L^{2}} \lesssim d_{q}(t) 2^{-q / 2}\|\nabla u(t)\|_{L^{2}}\|a(t)\|_{H^{2}}
$$

On the other hand, it follows from the product law in Besov spaces that

$$
\left\|\Delta_{q}(\nabla a \cdot \nabla u)(t)\right\|_{L^{2}} \lesssim d_{q}(t) 2^{q / 2}\|\nabla a(t)\|_{H^{1}}\|\nabla u(t)\|_{L^{2}}
$$

While (3.16) along with (3.17) yields

$$
\left\|\Delta_{q} T_{\nabla \Pi} a\right\|_{L^{2}}+\left\|\Delta_{q} \mathcal{R}(a, \nabla \Pi)\right\|_{L^{2}} \lesssim d_{q} 2^{q / 2}\|\nabla \Pi\|_{H^{-1}}\|a\|_{H^{2}},
$$

and a similar argument gives the same estimate for $\left\|\Delta_{q} T_{\nabla a} \Pi\right\|_{L^{2}}$.

Plugging (3.32-3.37) and (3.4) into (3.31), we arrive at

$$
\begin{aligned}
& \|u\|_{\widetilde{L}_{T}^{\infty}\left(B_{2,1}^{-1 / 2}\right)}+\left\|u^{H}\right\|_{L_{T}^{1}\left(B_{2,1}^{3 / 2}\right)} \\
& \leq C\left\{\left\|u_{0}\right\|_{B_{2,1}^{-1 / 2}}+\int_{0}^{T}\|u(t)\|_{B_{2,1}^{-1 / 2}}\|\nabla v(t)\|_{B_{2,1}^{3 / 2}} \mathrm{~d} t\right. \\
& \left.\quad+\|a\|_{L_{T}^{\infty}\left(H^{2}\right)}\left(\|\nabla \Pi\|_{L_{T}^{1}\left(H^{-1}\right)}+\|\nabla u\|_{L_{T}^{1}\left(L^{2}\right)}\right)+\|f\|_{L_{T}^{1}\left(B_{2,1}^{-1 / 2}\right)}\right\}
\end{aligned}
$$

which along with the fact that $\|u\|_{L_{T}^{1}\left(B_{2,1}^{3 / 2}\right)} \lesssim\left\|u^{H}\right\|_{L_{T}^{1}\left(B_{2,1}^{3 / 2}\right)}+\|u\|_{L_{T}^{1}\left(L^{2}\right)}$ leads to (3.29).

Remark 6. Applying Gronwall's inequality to (3.29) yields

$$
\begin{aligned}
& \|u\|_{\widetilde{L}_{T}^{\infty}\left(B_{2,1}^{-1 / 2}\right)}+\|u\|_{L_{T}^{1}\left(B_{2,1}^{3 / 2}\right)} \\
& \leqq \\
& \quad C \exp \left(C \int_{0}^{T}\|\nabla v(t)\|_{B_{2,1}^{3 / 2}} \mathrm{~d} t\right)\left\{\left\|u_{0}\right\|_{B_{2,1}^{-1 / 2}}\right. \\
& \left.\quad+\|u\|_{L_{T}^{1}\left(L^{2}\right)}+\|f\|_{L_{T}^{1}\left(B_{2,1}^{-1 / 2}\right)}+\|a\|_{L_{T}^{\infty}\left(H^{2}\right)}\left(\|\nabla \Pi\|_{L_{T}^{1}\left(H^{-1}\right)}+\|\nabla u\|_{L_{T}^{1}\left(L^{2}\right)}\right)\right\} .
\end{aligned}
$$




\section{Local Wellposedness of (1.2)}

The goal of this section is to prove the following local wellposedness result of (1.2):

Theorem 2. Under the assumptions of Theorem 1, a positive time $T$ exists such that (1.2) has a unique local solution $(a, u) \in \mathcal{C}_{b}\left([0, T] ; B_{2,1}^{3 / 2}\left(\mathbb{R}^{3}\right)\right) \times$ $\mathcal{C}_{b}\left([0, T] ; \dot{B}_{2,1}^{1 / 2}\left(\mathbb{R}^{3}\right)\right) \cap L^{1}\left([0, T] ; \dot{B}_{2,1}^{5 / 2}\left(\mathbb{R}^{3}\right)\right)$. Furthermore, there exits a small constant $c$, which depends on $\left\|a_{0}\right\|_{B_{2,1}^{3 / 2}}$ such that if

$$
\left\|u_{0}\right\|_{\dot{B}_{2,1}^{1 / 2}} \leqq c
$$

then $T \geqq 1$, and there holds

$$
\|u\|_{\widetilde{L}^{\infty}\left([0, T] ; \dot{B}_{2,1}^{1 / 2}\right)}+\int_{0}^{T}\|u(\tau)\|_{\dot{B}_{2,1}^{5 / 2}} d \tau \leqq C\left\|u_{0}\right\|_{\dot{B}_{2,1}^{1 / 2}} .
$$

Moreover, for any $t_{0}>0$, there holds

$$
\begin{aligned}
& \|u\|_{\widetilde{L}^{\infty}\left(\left[t_{0}, T\right] ; \dot{B}_{2,1}^{3 / 2}\right)}+\|u\|_{L^{1}\left(\left[t_{0}, T\right] ; \dot{B}_{2,1}^{7 / 2}\right)}+\|\nabla \Pi\|_{L^{1}\left(\left[t_{0}, T\right] ; \dot{B}_{2,1}^{3 / 2}\right)} \\
& \quad \leqq C\left(\left\|a_{0}\right\|_{\left.B_{2,1}^{3 / 2}\right)}\left\|u_{0}\right\|_{\dot{B}_{2,1}^{1 / 2}}\left(1+1 / \sqrt{t_{0}}\right) \exp \left\{C\left\|u_{0}\right\|_{\dot{B}_{2,1}^{1 / 2}}\right\}\right.
\end{aligned}
$$

Remark 7. Thanks to (4.1) and (4.2), there exists $t_{1} \in(0,1)$ such that $u\left(t_{1}\right) \in$ $\dot{B}_{2,1}^{1 / 2}\left(\mathbb{R}^{3}\right) \cap \dot{B}_{2,1}^{7 / 2}\left(\mathbb{R}^{3}\right)$ and satisfies $(1.8)$.

\subsection{Existence Part of Theorem 2}

We begin the existence proof by solving an approximate problem, then performing the uniform estimates for the approximate solutions thus obtained. Finally, the existence part of Theorem 1 is reached by a compactness argument.

Step 1: Construction of smooth approximate solutions

We first smooth out the initial data. For $n \in \mathbb{N}$, let

$$
a_{0}^{n} \stackrel{\text { def }}{=} \dot{S}_{n} a_{0}-\dot{S}_{-n} a_{0} \text { and } u_{0}^{n} \stackrel{\text { def }}{=} \dot{S}_{n} u_{0}-\dot{S}_{-n} u_{0},
$$

then $a_{0}^{n}, u_{0}^{n} \in H^{\infty}\left(\mathbb{R}^{3}\right)$, and Theorem 0.2 of [11] ensures that the system (1.2) with the initial data $\left(a_{0}^{n}, u_{0}^{n}\right)$ admits a unique local in time solution $\left(a^{n}, u^{n}, \nabla \Pi^{n}\right)$ satisfying

$$
\begin{aligned}
& a^{n} \in \mathcal{C}\left(\left[0, T^{n}\right) ; H^{s+1}\left(\mathbb{R}^{3}\right)\right), u^{n} \in \mathcal{C}\left(\left[0, T^{n}\right) ;\right. \\
& \left.\qquad H^{s}\left(\mathbb{R}^{3}\right)\right) \cap \widetilde{L}_{\mathrm{loc}}^{1}\left(\left[0, T^{n}\right) ; H^{s+2}\left(\mathbb{R}^{3}\right)\right) \\
& \text { and } \nabla \Pi^{n} \in L^{1}\left(\left[0, T^{n}\right) ; H^{s}\left(\mathbb{R}^{3}\right)\right) \text { with } s>1 / 2 .
\end{aligned}
$$

Step 2: Uniform estimates to the approximate solutions

Our first goal is to prove that there exists a positive time $0<T<\inf _{n \in \mathbb{N}} T^{n}$ such that $\left(a^{n}, u^{n}, \nabla \Pi^{n}\right)$ is uniformly bounded in the space

$$
E_{T} \stackrel{\text { def }}{=} \widetilde{L}_{T}^{\infty}\left(\dot{B}_{2,1}^{3 / 2}\right) \times\left(\widetilde{L}_{T}^{\infty}\left(\dot{B}_{2,1}^{1 / 2}\right) \cap L_{T}^{1}\left(\dot{B}_{2,1}^{5 / 2}\right)\right) \times L_{T}^{1}\left(\dot{B}_{2,1}^{1 / 2}\right)
$$


Let $u_{L}^{n}(t) \stackrel{\text { def }}{=} \mathrm{e}^{t \Delta} u_{0}^{n}$. Then it is easy to observe that

$$
\begin{aligned}
& \left\|u_{L}^{n}\right\|_{\widetilde{L}^{\infty}\left(\mathbb{R}^{+} ; \dot{B}_{2,1}^{1 / 2}\right)} \lesssim\left\|u_{0}\right\|_{\dot{B}_{2,1}^{1 / 2}} \text { and } \\
& \left\|u_{L}^{n}\right\|_{L_{t}^{1}\left(\dot{B}_{2,1}^{5 / 2}\right)} \lesssim \sum_{q \in \mathbb{Z}} 2^{q / 2}\left(1-\mathrm{e}^{-c t 2^{2 q}}\right)\left\|\dot{\Delta}_{q} u_{0}\right\|_{L^{2}} .
\end{aligned}
$$

Let $u^{n} \stackrel{\text { def }}{=} u_{L}^{n}+\bar{u}^{n}$. Then $\left(a^{n}, \bar{u}^{n}, \Pi^{n}\right)$ solves

$$
\left\{\begin{array}{l}
\partial_{t} a^{n}+\left(u_{L}^{n}+\bar{u}^{n}\right) \cdot \nabla a^{n}=0, \\
\partial_{t} \bar{u}^{n}+u_{L}^{n} \cdot \nabla \bar{u}^{n}-\left(1+a^{n}\right)\left(\Delta \bar{u}^{n}-\nabla \Pi^{n}\right)=H_{n}, \\
\operatorname{div} \bar{u}^{n}=0, \\
\left(a^{n}, \bar{u}^{n}\right)_{\mid t=0}=\left(a_{0}^{n}, 0\right),
\end{array}\right.
$$

with

$$
H_{n}=H\left(a^{n}, \bar{u}^{n}, u_{L}^{n}, \nabla \Pi_{L}^{n}\right)=-u_{L}^{n} \cdot \nabla u_{L}^{n}-\bar{u}^{n} \cdot \nabla u_{L}^{n}-\bar{u}^{n} \cdot \nabla \bar{u}^{n}+a^{n} \Delta u_{L}^{n} .
$$

Proposition 6. Let $a \in L_{T}^{\infty}\left(B_{2,1}^{3 / 2}\left(\mathbb{R}^{3}\right)\right), u_{0} \in \dot{B}_{2,1}^{1 / 2}\left(\mathbb{R}^{3}\right)$, which satisfy $1+a \geqq \underline{b}$ for some positive constant $\underline{b}$ and $\operatorname{div} u_{0}=0$. Let $f$ belong to $L_{T}^{1}\left(\dot{B}_{2,1}^{1 / 2}\right)$ with $\operatorname{div} f \in L_{T}^{1}\left(\dot{H}^{-1}\right), v \in L_{T}^{1}\left(\dot{B}_{2,1}^{5 / 2}\right) \cap L_{T}^{\infty}\left(\dot{B}_{2,1}^{1 / 2}\right)$, and $(u, \nabla \Pi) \in\left(\mathcal{C}\left([0, T] ; B_{2,1}^{1 / 2}\right) \cap\right.$ $\left.L_{\text {loc }}^{1}\left((0, T) ; \dot{B}_{2,1}^{5 / 2}\right)\right) \times L_{\text {loc }}^{1}\left((0, T) ; \dot{B}_{2,1}^{1 / 2}\right)$, which solve

$$
\left\{\begin{array}{l}
\partial_{t} u+(v \cdot \nabla) u-(1+a)(\Delta u-\nabla \Pi)=f, \\
\operatorname{div} u=0 \\
\left.u\right|_{t=0}=u_{0}
\end{array}\right.
$$

Then there holds for $t \in(0, T]$

$$
\begin{aligned}
& \|u\|_{\widetilde{L}_{t}^{\infty}\left(\dot{B}_{2,1}^{1 / 2}\right)}+\|u\|_{L_{t}^{1}\left(\dot{B}_{2,1}^{5 / 2}\right)}+\|\nabla \Pi\|_{L_{t}^{1}\left(B_{2,1}^{1 / 2}\right)} \\
& \leqq C\left(\left\|u_{0}\right\|_{\dot{B}_{2,1}^{1 / 2}}+\|f\|_{L_{t}^{1}\left(\dot{B}_{2,1}^{1 / 2}\right)}+2^{2 m}\|a\|_{L_{t}^{\infty}\left(L^{2}\right)}\|\operatorname{div} f\|_{L_{t}^{1}\left(\dot{H}^{-1}\right)}\right.
\end{aligned}
$$

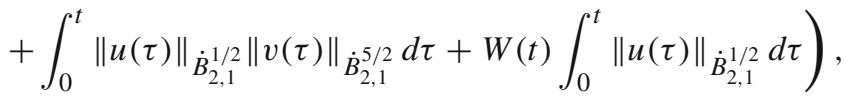

with

$$
W(t) \stackrel{\text { def }}{=} 2^{8 m}\|a\|_{L_{t}^{\infty}\left(L^{2}\right)}^{4}\left(1+\|a\|_{L_{t}^{\infty}\left(L^{\infty}\right)}^{4}+\|v\|_{L_{t}^{\infty}\left(\dot{B}_{2,1}^{1 / 2}\right)}^{4}\right)+2^{2 m}\|a\|_{L_{t}^{\infty}\left(L^{\infty}\right)}^{2},
$$

provided that

$$
\left\|a-\dot{S}_{m} a\right\|_{L_{T}^{\infty}\left(\dot{B}_{2,1}^{3 / 2}\right)} \leqq c
$$

for some sufficiently small positive constant $c$ and some integer $m \in \mathbb{Z}$. 
Proof. We first deduce from (4.7) for $c$ sufficiently small and $\underline{b} \leqq 1+a$ that

$$
1+\dot{S}_{m} a=1+a+\left(\dot{S}_{m} a-a\right) \geqq \frac{b}{2} .
$$

Then we split $a$ as $\dot{S}_{m} a+\left(a-\dot{S}_{m} a\right)$ so that (4.5) reads

$\partial_{t} u+(v \cdot \nabla) u-\left(1+\dot{S}_{m} a\right) \Delta u+\nabla\left(\left(1+\dot{S}_{m} a\right) \Pi\right)=E_{m}+\Pi \nabla \dot{S}_{m} a+f$

with $E_{m}=\left(a-\dot{S}_{m} a\right)(\Delta u-\nabla \Pi)$.

Step 1. The estimate of $\|u\|_{\widetilde{L}_{t}^{\infty}\left(\dot{B}_{2,1}^{1 / 2}\right)}+\|u\|_{L_{t}^{1}\left(\dot{B}_{2,1}^{5 / 2}\right)}$.

Applying $\dot{\Delta}_{q}$ to (4.9), and then a standard commutator's process, gives

$$
\begin{aligned}
& \partial_{t} \dot{\Delta}_{q} u+(v \cdot \nabla) \dot{\Delta}_{q} u-\operatorname{div}\left\{\left(1+\dot{S}_{m} a\right) \dot{\Delta}_{q} \nabla u\right\}+\dot{\Delta}_{q} \nabla\left\{\left(1+\dot{S}_{m} a\right) \Pi\right\} \\
& =\dot{\Delta}_{q} f+\dot{\Delta}_{q} E_{m}+\dot{\Delta}_{q}\left(\Pi \nabla \dot{S}_{m} a\right) \\
& \quad+\left[\dot{\Delta}_{q}, v \cdot \nabla\right] u+\operatorname{div}\left[\dot{\Delta}_{q}, \dot{S}_{m} a\right] \nabla u-\dot{\Delta}_{q}\left(\nabla \dot{S}_{m} a \cdot \nabla u\right) .
\end{aligned}
$$

Taking the $L^{2}$ inner product of the above equation with $\dot{\Delta}_{q} u$ and using $\operatorname{div} v=$ $\operatorname{div} u=0$, we obtain

$$
\begin{aligned}
& \frac{1}{2} \frac{\mathrm{d}}{\mathrm{d} t}\left\|\dot{\Delta}_{q} u\right\|_{L^{2}}^{2}+\int_{\mathbb{R}^{3}}\left(1+\dot{S}_{m} a\right)\left|\dot{\Delta}_{q} \nabla u\right|^{2} \mathrm{~d} x \\
& \leq\left\|\dot{\Delta}_{q} u\right\|_{L^{2}}\left\{\left\|\dot{\Delta}_{q} f\right\|_{L^{2}}+\left\|\dot{\Delta}_{q} E_{m}\right\|_{L^{2}}+\left\|\dot{\Delta}_{q}\left(\Pi \nabla \dot{S}_{m} a\right)\right\|_{L^{2}}\right. \\
& \left.\quad+\left\|\left[\dot{\Delta}_{q}, v \cdot \nabla\right] u\right\|_{L^{2}}+2^{q}\left\|\left[\dot{\Delta}_{q}, \dot{S}_{m} a\right] \nabla u\right\|_{L^{2}}+\left\|\dot{\Delta}_{q}\left(\nabla \dot{S}_{m} a \cdot \nabla u\right)\right\|_{L^{2}}\right\}
\end{aligned}
$$

which along with Lemma 1 and (4.8) ensures that

$$
\begin{aligned}
& \|u\|_{\widetilde{L}_{t}^{\infty}\left(\dot{B}_{2,1}^{1 / 2}\right)}+\kappa \underline{b}\|u\|_{L_{t}^{1}\left(\dot{B}_{2,1}^{5 / 2}\right)} \lesssim\left\|u_{0}\right\|_{\dot{B}_{2,1}^{1 / 2}}+\|f\|_{L_{t}^{1}\left(\dot{B}_{2,1}^{1 / 2}\right)} \\
& +\left\|E_{m}\right\|_{L_{t}^{1}\left(\dot{B}_{2,1}^{1 / 2}\right)}+\left\|\Pi \nabla \dot{S}_{m} a\right\|_{L_{t}^{1}\left(\dot{B}_{2,1}^{1 / 2}\right)}+\sum_{q \in \mathbb{Z}} 2^{q / 2}\left\|\left[\dot{\Delta}_{q}, v \cdot \nabla\right] u\right\|_{L_{t}^{1}\left(L^{2}\right)} \\
& +\sum_{q \in \mathbb{Z}} 2^{3 q / 2}\left\|\left[\dot{\Delta}_{q}, \dot{S}_{m} a\right] \nabla u\right\|_{L_{t}^{1}\left(L^{2}\right)}+\left\|\nabla \dot{S}_{m} a \cdot \nabla u\right\|_{L_{t}^{1}\left(\dot{B}_{2,1}^{1 / 2}\right)}
\end{aligned}
$$

for some positive constant $\kappa$. Applying product laws in Besov spaces and (3.1) yields

$$
\begin{aligned}
& \left\|E_{m}\right\|_{L_{t}^{1}\left(\dot{B}_{2,1}^{1 / 2}\right)} \lesssim\left\|a-\dot{S}_{m} a\right\|_{L_{t}^{\infty}\left(\dot{B}_{2,1}^{3 / 2}\right)}\|\Delta u-\nabla \Pi\|_{L_{t}^{1}\left(\dot{B}_{2,1}^{1 / 2}\right)}, \\
& \left\|\Pi \nabla \dot{S}_{m} a\right\|_{L_{t}^{1}\left(\dot{B}_{2,1}^{1 / 2}\right)}+\left\|\nabla \dot{S}_{m} a \cdot \nabla u\right\|_{L_{t}^{1}\left(\dot{B}_{2,1}^{1 / 2}\right)} \\
& \quad \lesssim\left(\|\nabla \Pi\|_{L_{t}^{1}\left(L^{2}\right)}+\|\nabla u\|_{L_{t}^{1}\left(\dot{H}^{1}\right)}\right)\left\|\nabla \dot{S}_{m} a\right\|_{L_{t}^{\infty}\left(\dot{H}^{1}\right)} \\
& \quad \lesssim 2^{2 m}\|a\|_{L_{t}^{\infty}\left(L^{2}\right)}\left(\|u\|_{L_{t}^{1}\left(\dot{H}^{2}\right)}+\|\nabla \Pi\|_{L_{t}^{1}\left(L^{2}\right)}\right), \\
& \sum_{q \in \mathbb{Z}} 2^{q / 2}\left\|\left[\dot{\Delta}_{q}, v \cdot \nabla\right] u\right\|_{L_{t}^{1}\left(L^{2}\right)} \lesssim \int_{0}^{t}\|v\|_{\dot{B}_{2,1}^{5 / 2}}\|u\|_{\dot{B}_{2,1}^{1 / 2}} \mathrm{~d} \tau
\end{aligned}
$$


and

$$
\begin{aligned}
& \sum_{q \in \mathbb{Z}} 2^{3 q / 2}\left\|\left[\dot{\Delta}_{q}, \dot{S}_{m} a\right] \nabla u\right\|_{L_{t}^{1}\left(L^{2}\right)} \\
& \quad \lesssim 2^{m}\|a\|_{L_{t}^{\infty}\left(L^{\infty}\right)}\|u\|_{L_{t}^{1}\left(\dot{B}_{2,1}^{3 / 2}\right)}+2^{2 m}\|a\|_{L_{t}^{\infty}\left(L^{2}\right)}\|u\|_{L_{t}^{1}\left(\dot{H}^{2}\right)}
\end{aligned}
$$

Whence we obtain

$$
\begin{aligned}
& \|u\|_{\widetilde{L}_{t}^{\infty}\left(\dot{B}_{2,1}^{1 / 2}\right)}+\|u\|_{L_{t}^{1}\left(\dot{B}_{2,1}^{5 / 2}\right)} \lesssim\left\|u_{0}\right\|_{\dot{B}_{2,1}^{1 / 2}}+2^{m}\|a\|_{L_{t}^{\infty}\left(L^{\infty}\right)}\|u\|_{L_{t}^{1}\left(\dot{B}_{2,1}^{3 / 2}\right)} \\
& \quad+\left\|a-\dot{S}_{m} a\right\|_{L_{t}^{\infty}\left(\dot{B}_{2,1}^{3 / 2}\right)}\left(\|u\|_{L_{t}^{1}\left(\dot{B}_{2,1}^{5 / 2}\right)}+\|\nabla \Pi\|_{L_{t}^{1}\left(\dot{B}_{2,1}^{1 / 2}\right)}\right)+\|f\|_{L_{t}^{1}\left(\dot{B}_{2,1}^{1 / 2}\right)} \\
& \quad+2^{2 m}\|a\|_{L_{t}^{\infty}\left(L^{2}\right)}\left(\|u\|_{L_{t}^{1}\left(\dot{H}^{2}\right)}+\|\nabla \Pi\|_{L_{t}^{1}\left(L^{2}\right)}\right)+\int_{0}^{t}\|v\|_{\dot{B}_{2,1}^{5 / 2}}\|u\|_{\dot{B}_{2,1}^{1 / 2}} \mathrm{~d} \tau .
\end{aligned}
$$

Step 2. The estimate of $\|\nabla \Pi\|_{L_{t}^{1}\left(\dot{B}_{2,1}^{1 / 2}\right)}$.

Thanks to $\operatorname{div} u=0$, we get by applying div to (4.5) that

$$
\begin{aligned}
& \operatorname{div}\left(\left(1+\dot{S}_{m} a\right) \nabla \Pi\right)=-\operatorname{div}(v \cdot \nabla u)+\nabla \dot{S}_{m} a \cdot \Delta u \\
& \quad+\operatorname{div}\left(\left(\dot{S}_{m} a-a\right)(\nabla \Pi-\Delta u)\right)+\operatorname{div} f .
\end{aligned}
$$

Applying $\dot{\Delta}_{q}$ to the above equation and taking the $L^{2}$ inner product of the resulting equation with $\dot{\Delta}_{q} \Pi$, we infer from (4.8) that

$$
\begin{aligned}
& \underline{b}\left\|\dot{\Delta}_{q} \nabla \Pi\right\|_{L^{2}}^{2} \lesssim\left\|\dot{\Delta}_{q} \nabla \Pi\right\|_{L^{2}}\left(\left\|\dot{\Delta}_{q} f\right\|_{L^{2}}+\left\|\dot{\Delta}_{q}\left(\left(\dot{S}_{m} a-a\right) \nabla \Pi\right)\right\|_{L^{2}}\right. \\
& \quad+2^{-q}\left\|\dot{\Delta}_{q}\left(\nabla \dot{S}_{m} a \cdot \Delta u\right)\right\|_{L^{2}}+\left\|\Delta_{q}(u \cdot \nabla v)\right\|_{L^{2}} \\
& \left.\quad+\left\|\dot{\Delta}_{q}\left(\left(\dot{S}_{m} a-a\right) \Delta u\right)\right\|_{L^{2}}+\left\|\left[\dot{\Delta}_{q}, \dot{S}_{m} a\right] \nabla \Pi\right\|_{L^{2}}\right),
\end{aligned}
$$

where we used the fact that $\operatorname{div} v=\operatorname{div} u=0$ so that $\operatorname{div}(v \cdot \nabla u)=\operatorname{div}(u \cdot \nabla v)$. This leads to

$$
\begin{aligned}
& \|\nabla \Pi\|_{\dot{B}_{2,1}^{1 / 2}} \lesssim\left\|\nabla \dot{S}_{m} a \cdot \Delta u\right\|_{\dot{B}_{2,1}^{-1 / 2}}+\left\|\left(\dot{S}_{m} a-a\right) \nabla \Pi\right\|_{\dot{B}_{2,1}^{1 / 2}}+\|f\|_{\dot{B}_{2,1}^{1 / 2}} \\
& \quad+\left\|\left(\dot{S}_{m} a-a\right) \Delta u\right\|_{\dot{B}_{2,1}^{1 / 2}}+\|v\|_{\dot{B}_{2,1}^{5 / 2}}\|u\|_{\dot{B}_{2,1}^{1 / 2}}+\sum_{q \in \mathbb{Z}} 2^{q / 2}\left\|\left[\dot{\Delta}_{q}, \dot{S}_{m} a\right] \nabla \Pi\right\|_{L^{2}} \\
& \lesssim\left\|\dot{S}_{m} a\right\|_{\dot{H}^{2}}\left(\|u\|_{\dot{H}^{2}}+\|\nabla \Pi\|_{L^{2}}\right) \\
& \quad+\left\|\dot{S}_{m} a-a\right\|_{\dot{B}_{2,1}^{3 / 2}}\left(\|u\|_{\dot{B}_{2,1}^{5 / 2}}+\|\nabla \Pi\|_{\dot{B}_{2,1}^{1 / 2}}+\|v\|_{\dot{B}_{2,1}^{5 / 2}}\|u\|_{\dot{B}_{2,1}^{1 / 2}}+\|f\|_{\dot{B}_{2,1}^{1 / 2}}\right.
\end{aligned}
$$

which along with (4.7) and Lemma 1 implies that

$$
\begin{aligned}
& \|\nabla \Pi\|_{L_{t}^{1}\left(\dot{B}_{2,1}^{1 / 2}\right)} \lesssim\|f\|_{L_{t}^{1}\left(\dot{B}_{2,1}^{1 / 2}\right)}+2^{2 m}\|a\|_{L_{t}^{\infty}\left(L^{2}\right)}\left(\|u\|_{L_{t}^{1}\left(\dot{H}^{2}\right)}+\|\nabla \Pi\|_{L_{t}^{1}\left(L^{2}\right)}\right) \\
& \quad+\left\|a-\dot{S}_{m} a\right\|_{L_{t}^{\infty}\left(\dot{B}_{2,1}^{3 / 2}\right)}\|u\|_{L_{t}^{1}\left(\dot{B}_{2,1}^{5 / 2}\right)}+\int_{0}^{t}\|u(\tau)\|_{\dot{B}_{2,1}^{1 / 2}}\|v(\tau)\|_{\dot{B}_{2,1}^{5 / 2}} \mathrm{~d} \tau .
\end{aligned}
$$

On the other hand, it is easy to observe from (4.5) that

$$
\begin{aligned}
\underline{b}\|\nabla \Pi\|_{L^{2}} & \lesssim\left(\|v\|_{L^{3}}+\|a\|_{L^{\infty}}\right)\|\Delta u\|_{L^{2}}+\|\operatorname{div} f\|_{\dot{H}^{-1}} \\
& \lesssim\left(\|v\|_{\dot{B}_{2,1}^{1 / 2}}+\|a\|_{L^{\infty}}\right)\|u\|_{\dot{B}_{2,1}^{1 / 2}}^{1 / 4}\|u\|_{\dot{B}_{2,1}^{5 / 2}}^{3 / 4}+\|\operatorname{div} f\|_{\dot{H}^{-1}},
\end{aligned}
$$


which gives rise to

$$
\begin{aligned}
& \|\nabla \Pi\|_{L_{t}^{1}\left(\dot{B}_{2,1}^{1 / 2}\right)} \lesssim\left\|a-\dot{S}_{m} a\right\|_{L_{t}^{\infty}\left(\dot{B}_{2,1}^{3 / 2}\right)}\|u\|_{L_{t}^{1}\left(\dot{B}_{2,1}^{5 / 2}\right)}+\|f\|_{L_{t}^{1}\left(\dot{B}_{2,1}^{1 / 2}\right)} \\
& \quad+2^{2 m}\|a\|_{L_{t}^{\infty}\left(L^{2}\right)}\|\operatorname{div} f\|_{\dot{H}^{-1}}+\int_{0}^{t}\|u\|_{\dot{B}_{2,1}^{1 / 2}\|v\|_{\dot{B}_{2,1}^{5 / 2}} \mathrm{~d} \tau} \\
& \quad+2^{2 m}\|a\|_{L_{t}^{\infty}\left(L^{2}\right)}\left(1+\|v\|_{L_{t}^{\infty}\left(\dot{B}_{2,1}^{1 / 2}\right)}+\|a\|_{L_{t}^{\infty}\left(L^{\infty}\right)}\right)\|u\|_{L_{t}^{1}\left(\dot{H}^{2}\right)} .
\end{aligned}
$$

This along with Lemma 1, (4.7) and (4.11) ensures that

$$
\begin{aligned}
& \|u\|_{\widetilde{L}_{t}^{\infty}\left(\dot{B}_{2,1}^{1 / 2}\right)}+\|u\|_{L_{t}^{1}\left(\dot{B}_{2,1}^{5 / 2}\right)}+\|\nabla \Pi\|_{L_{t}^{1}\left(\dot{B}_{2,1}^{1 / 2}\right)} \\
& \lesssim\left\|u_{0}\right\|_{\dot{B}_{2,1}^{1 / 2}}+\int_{0}^{t}\|u(\tau)\|_{\dot{B}_{2,1}^{1 / 2}}\|v(\tau)\|_{\dot{B}_{2,1}^{5 / 2}} \mathrm{~d} \tau+2^{2 m}\|a\|_{L_{t}^{\infty}\left(L^{2}\right)}\|\operatorname{div} f\|_{\dot{H}^{-1}} \\
& \quad+2^{2 m}\|a\|_{L_{t}^{\infty}\left(L^{2}\right)}\left(1+\|v\|_{L_{t}^{\infty}\left(\dot{B}_{2,1}^{1 / 2}\right)}+\|a\|_{L_{t}^{\infty}\left(L^{\infty}\right)}\right)\|u\|_{L_{t}^{1}\left(\dot{B}_{2,1}^{1 / 2}\right)}^{1 / 4}\|u\|_{L_{t}^{1}\left(\dot{B}_{2,1}^{5 / 2}\right)}^{3 / 4} \\
& \quad+\|f\|_{L_{t}^{1}\left(\dot{B}_{2,1}^{1 / 2}\right)}+2^{m}\|a\|_{L_{t}^{\infty}\left(L^{\infty}\right)}\|u\|_{L_{t}^{1}\left(\dot{B}_{2,1}^{1 / 2}\right)}^{1 / 2}\|u\|_{L_{t}^{1}\left(\dot{B}_{2,1}^{5 / 2}\right)}^{1 / 2}
\end{aligned}
$$

From this, using Young's inequality, we deduce that

$$
\begin{aligned}
& \|u\|_{\widetilde{L}_{t}^{\infty}\left(\dot{B}_{2,1}^{1 / 2}\right)}+\|u\|_{L_{t}^{1}\left(\dot{B}_{2,1}^{5 / 2}\right)}+\|\nabla \Pi\|_{L_{t}^{1}\left(\dot{B}_{2,1}^{1 / 2}\right)} \\
& \lesssim\left\|u_{0}\right\|_{\dot{B}_{2,1}^{1 / 2}}+\|f\|_{L_{t}^{1}\left(\dot{B}_{2,1}^{1 / 2}\right)}+2^{2 m}\|a\|_{L_{t}^{\infty}\left(L^{2}\right)}\|\operatorname{div} f\|_{\dot{H}^{-1}}
\end{aligned}
$$

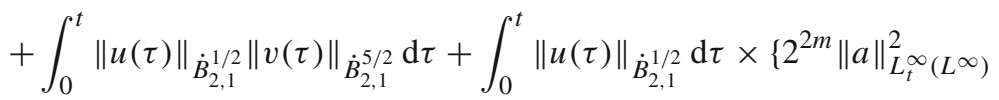

$$
\begin{aligned}
& \left.+2^{8 m}\|a\|_{L_{t}^{\infty}\left(L^{2}\right)}^{4}\left(1+\|a\|_{L_{t}^{\infty}\left(L^{\infty}\right)}^{4}+\|v\|_{L_{t}^{\infty}\left(\dot{B}_{2,1}^{1 / 2}\right)}^{4}\right)\right\} .
\end{aligned}
$$

This leads to (4.6).

Remark 8. Notice that if $\operatorname{div} u=0$,

$$
\sum_{i, j=1}^{3} \partial_{i} u_{j} \partial_{j} u_{i}=\sum_{i, j=1}^{3} \dot{T}_{\partial_{i} u_{j}} \partial_{j} u_{i}+\sum_{i, j=1}^{3} \partial_{i} \dot{R}\left(u_{j}, \partial_{j} u_{i}\right),
$$

which leads to

$$
\left\|\sum_{i, j=1}^{3} \partial_{i} u_{j} \partial_{j} u_{i}\right\|_{\dot{B}_{2,1}^{-1 / 2}} \lesssim\|\nabla u\|_{L^{\infty}\|u\|_{\dot{B}_{2,1}^{1 / 2}}}
$$

Whence in particular, when $v=u$ in (4.5), using the above estimate in (4.12) and following the same line as the proof of (4.6), one deduces that for any $t \in(0, T]$,

$$
\begin{aligned}
& \|u\|_{\widetilde{L}_{t}^{\infty}\left(\dot{B}_{2,1}^{1 / 2}\right)}+\|u\|_{L_{t}^{1}\left(\dot{B}_{2,1}^{5 / 2}\right)}+\|\nabla \Pi\|_{L_{t}^{1}\left(\dot{B}_{2,1}^{1 / 2}\right)} \\
& \leq C\left(\left\|u_{0}\right\|_{\dot{B}_{2,1}^{1 / 2}}+\int_{0}^{t}\left(\|\nabla u(\tau)\|_{L^{\infty}}+W(\tau)\right)\|u(\tau)\|_{\dot{B}_{2,1}^{1 / 2}} \mathrm{~d} \tau\right. \\
& \left.\quad+2^{2 m}\|a\|_{L_{t}^{\infty}\left(L^{2}\right)}\|\operatorname{div} f\|_{L_{t}^{1}\left(\dot{H}^{-1}\right)}+\|f\|_{L_{t}^{1}\left(\dot{B}_{2,1}^{1 / 2}\right)}\right)
\end{aligned}
$$


with

$$
W(t) \stackrel{\text { def }}{=} 2^{8 m}\|a\|_{L_{t}^{\infty}\left(L^{2}\right)}^{4}\left(1+\|a\|_{L_{t}^{\infty}\left(L^{\infty}\right)}^{4}+\|u\|_{L_{t}^{\infty}\left(\dot{B}_{2,1}^{1 / 2}\right)}^{4}\right)+2^{2 m}\|a\|_{L_{t}^{\infty}\left(L^{\infty}\right)}^{2} .
$$

Now let us turn to the uniform estimates of $\left(a^{n}, \bar{u}^{n}\right)$ obtained by solving (4.4). Firstly, as $a_{0} \in B_{2,1}^{3 / 2}$, we define $m \in \mathbb{Z}$ by

$$
m \stackrel{\text { def }}{=} \inf \left\{p \in \mathbb{Z} \mid \sum_{q \geqq p} 2^{3 q / 2}\left\|\Delta_{q} a_{0}\right\|_{L^{2}} \leqq c_{0} \underline{b}\right\}
$$

for some sufficiently small positive constant $c_{0}$.

Noticing that $\operatorname{div}\left(\bar{u}^{n}+u_{L}^{n}\right)=0$, we get by applying Proposition 1 to the first equation of (4.4) that

$$
\begin{aligned}
& \left\|a^{n}\right\|_{L_{t}^{\infty}\left(\dot{B}_{2,1}^{3 / 2}\right)} \lesssim\left\|a_{0}\right\|_{\dot{B}_{2,1}^{3 / 2}} \exp \left\{C\left(\left\|u_{L}^{n}\right\|_{L_{t}^{1}\left(\dot{B}_{2,1}^{5 / 2}\right)}+\left\|\bar{u}^{n}\right\|_{L_{t}^{1}\left(\dot{B}_{2,1}^{5 / 2}\right)}\right)\right\} \text { and } \\
& \left\|a^{n}\right\|_{L_{t}^{\infty}\left(L^{2}\right)} \leqq\left\|a_{0}\right\|_{L^{2}}, \quad\left\|a^{n}\right\|_{L_{t}^{\infty}\left(L^{\infty}\right)} \leqq\left\|a_{0}\right\|_{L^{\infty}}
\end{aligned}
$$

which leads to

$$
\begin{aligned}
W(t) & =2^{8 m}\left\|a^{n}\right\|_{L_{t}^{\infty}\left(L^{2}\right)}^{4}\left(1+\left\|a^{n}\right\|_{L_{t}^{\infty}\left(L^{\infty}\right)}^{4}+\left\|u_{L}^{n}\right\|_{L_{t}^{\infty}\left(\dot{B}_{2,1}^{1 / 2}\right)}^{4}\right)+2^{2 m}\left\|a^{n}\right\|_{L_{t}^{\infty}\left(L^{\infty}\right)}^{2} \\
& \leqq 2^{8 m}\left\|a_{0}\right\|_{L^{2}}^{4}\left(1+\left\|a_{0}\right\|_{L^{\infty}}^{4}+\left\|u_{0}\right\|_{\dot{B}_{2,1}^{1 / 2}}^{4}\right)+2^{2 m}\left\|a_{0}\right\|_{L^{\infty}}^{2} \stackrel{\text { def }}{=} N_{m}
\end{aligned}
$$

Noticing that $\|f g\|_{\dot{H}^{-1}} \lesssim\|f\|_{\dot{B}_{2,1}^{1 / 2}}\|g\|_{\dot{B}_{2,1}^{0}}$ and

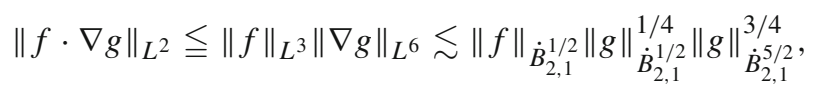

we have

$$
\begin{aligned}
& \left\|\bar{u}^{n} \cdot \nabla \bar{u}^{n}\right\|_{L^{2}} \lesssim\left\|\bar{u}^{n}\right\|_{\dot{B}_{2,1}^{1 / 2}}^{5 / 4}\left\|\bar{u}^{n}\right\|_{\dot{B}_{2,1}^{5 / 2}}^{3 / 4}, \quad\left\|u_{L}^{n} \cdot \nabla u_{L}^{n}\right\|_{L^{2}} \lesssim\left\|u_{L}^{n}\right\|_{\dot{B}_{2,1}^{1 / 2}}^{5 / 4}\left\|u_{L}^{n}\right\|_{\dot{B}_{2,1}^{5 / 2}}^{3 / 4} \\
& \left\|\bar{u}^{n} \cdot \nabla u_{L}^{n}\right\|_{L^{2}} \lesssim\left\|\bar{u}^{n}\right\|_{\dot{B}_{2,1}^{1 / 2}}^{1 / 2}\left\|\nabla u_{L}^{n}\right\|_{\dot{B}_{2,1}^{1 / 2}}^{1 / 4}\left\|\nabla u_{L}^{n}\right\|_{\dot{B}_{2,1}^{5 / 2}}^{3 / 4} \text { and } \\
& \left\|\nabla a^{n} \cdot \Delta u_{L}^{n}\right\|_{\dot{H}^{-1}} \lesssim\left\|a^{n}\right\|_{\dot{B}_{2,1}^{3 / 2}}^{3 / \|} u_{L}^{n}\left\|_{\dot{B}_{2,1}^{1 / 2}}^{1 / 4}\right\| u_{L}^{n} \|_{\dot{B}_{2,1}^{5 / 2}}^{3 / 4},
\end{aligned}
$$

so that $H_{n}$ defined in (4.4) satisfies

$$
\begin{aligned}
& \left\|\operatorname{div} H_{n}\right\|_{L_{t}^{1}\left(\dot{H}^{-1}\right)} \\
& \lesssim \int_{0}^{t}\left(\left\|\bar{u}^{n} \cdot \nabla \bar{u}^{n}\right\|_{L^{2}}+\left\|\bar{u}^{n} \cdot \nabla u_{L}^{n}\right\|_{L^{2}}+\left\|u_{L}^{n} \cdot \nabla u_{L}^{n}\right\|_{L^{2}}+\left\|\nabla a^{n} \cdot \Delta u_{L}^{n}\right\|_{\dot{H}^{-1}}\right) \mathrm{d} \tau \\
& \lesssim \int_{0}^{t}\left(\left\|\bar{u}^{n}\right\|_{\dot{B}_{2,1}^{1 / 2}}^{5 / 4}\left\|\bar{u}^{n}\right\|_{\dot{B}_{2,1}^{5 / 2}}^{3 / 4}+\left\|\bar{u}^{n}\right\|_{\dot{B}_{2,1}^{1 / 2}\left\|u_{L}^{n}\right\|_{\dot{B}_{2,1}^{1 / 2}}^{1 / 4}\left\|u_{L}^{n}\right\|_{\dot{B}_{2,1}^{5 / 2}}^{3 / 4}}\right. \\
& \left.\quad+\left\|u_{L}^{n}\right\|_{\dot{B}_{2,1}^{1 / 2}}^{5 / 4}\left\|u_{L}^{n}\right\|_{\dot{B}_{2,1}^{5 / 2}}^{3 / 4}\right) \mathrm{d} \tau+\int_{0}^{t}\left\|a^{n}\right\|_{\dot{B}_{2,1}^{3 / 2}}^{3 / \|} u_{L}^{n}\left\|_{\dot{B}_{2,1}^{1 / 2}}^{1 / 4}\right\| u_{L}^{n} \|_{\dot{B}_{2,1}^{5 / 2}}^{3 / 4} \mathrm{~d} \tau .
\end{aligned}
$$


Applying Young's inequality yields for any $\eta>0$

$$
\begin{aligned}
& 2^{2 m}\left\|\dot{S}_{m} a\right\|_{L_{t}^{\infty}\left(L^{2}\right)}\left\|\operatorname{div} H_{n}\right\|_{L_{t}^{1}\left(\dot{H}^{-1}\right)} \\
& \lesssim\left\|u_{L}^{n}\right\|_{L_{t}^{1}\left(\dot{B}_{2,1}^{5 / 2}\right)}+C_{\eta} 2^{8 m}\left\|\dot{S}_{m} a\right\|_{L_{t}^{\infty}\left(L^{2}\right)}^{4} \int_{0}^{t}\left\|\bar{u}^{n}\right\|_{\dot{B}_{2,1}^{1 / 2}}^{5} \mathrm{~d} \tau \\
& \quad+2^{8 m}\left\|\dot{S}_{m} a\right\|_{L_{t}^{\infty}\left(L^{2}\right)}^{4}\left(\int_{0}^{t}\left\|u_{L}^{n}\right\|_{\dot{B}_{2,1}^{1 / 2}}^{5} \mathrm{~d} \tau+\int_{0}^{t}\left\|a^{n}\right\|_{\dot{B}_{2,1}^{3 / 2}}^{4}\left\|u_{L}^{n}\right\|_{\dot{B}_{2,1}^{1 / 2}}\right) \mathrm{d} \tau \\
& \quad+2^{8 m}\left\|\dot{S}_{m} a\right\|_{L_{t}^{\infty}\left(L^{2}\right)}^{4} \int_{0}^{t}\left\|\bar{u}^{n}\right\|_{\dot{B}_{2,1}^{1 / 2}}^{4}\left\|u_{L}^{n}\right\|_{\dot{B}_{2,1}^{1 / 2}} \mathrm{~d} \tau+\eta\left\|\bar{u}^{n}\right\|_{L_{t}^{1}\left(\dot{B}_{2,1}^{5 / 2}\right)}
\end{aligned}
$$

Applying product laws in Besov spaces gives

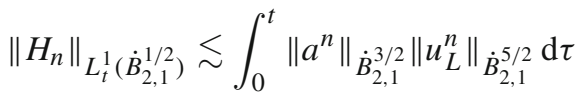

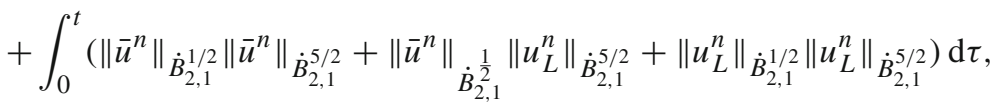

from which, with (4.3) and (4.17), we infer

$$
\begin{aligned}
& \left\|H_{n}\right\|_{L_{t}^{1}\left(\dot{B}_{2,1}^{1 / 2}\right)}+2^{2 m}\left\|\dot{S}_{m} a\right\|_{L_{t}^{\infty}\left(L^{2}\right)}\left\|\operatorname{div} H_{n}\right\|_{L_{t}^{1}\left(\dot{H}^{-1}\right)} \\
& \leqq \\
& \leq\left\|\bar{u}^{n}\right\|_{L_{t}^{1}\left(\dot{B}_{2,1}^{5 / 2}\right)}+C \int_{0}^{t}\left(\left\|\bar{u}^{n}\right\|_{\dot{B}_{2,1}^{1 / 2}\left\|\bar{u}^{n}\right\|_{\dot{B}_{2,1}^{5 / 2}}+\left\|\bar{u}^{n}\right\|_{\dot{B}_{2,1}^{\frac{1}{2}}}\left\|u_{L}^{n}\right\|_{\left.\dot{B}_{2,1}^{5 / 2}\right)} \mathrm{d} \tau}\right. \\
& \quad+C 2^{8 m}\left\|a_{0}\right\|_{L^{2}}^{4}\left\|u_{0}\right\|_{\dot{B}_{2,1}^{1 / 2}}\left(\int_{0}^{t}\left\|\bar{u}^{n}\right\|_{\dot{B}_{2,1}^{1 / 2}}^{4} \mathrm{~d} \tau+t\left\|u_{0}\right\|_{\dot{B}_{2,1}^{1 / 2}}^{4}+t\left\|a^{n}\right\|_{L_{t}^{\infty}\left(\dot{B}_{2,1}^{3 / 2}\right)}^{4}\right) \\
& \quad+C_{\eta} 2^{8 m}\left\|a_{0}\right\|_{L^{2}}^{4} \int_{0}^{t}\left\|\bar{u}^{n}\right\|_{\dot{B}_{2,1}^{1 / 2}}^{5} \mathrm{~d} \tau+C\left(\left\|a^{n}\right\|_{L_{t}^{\infty}\left(\dot{B}_{2,1}^{3 / 2}\right)}\right. \\
& \quad+\left\|u_{0}\right\|_{\left.\dot{B}_{2,1}^{1 / 2}\right)}\left\|u_{L}^{n}\right\|_{L_{t}^{1}\left(\dot{B}_{2,1}^{5 / 2}\right)}+C\left\|u_{L}^{n}\right\|_{L_{t}^{1}\left(\dot{B}_{2,1}^{5 / 2}\right)} .
\end{aligned}
$$

Let $Z^{n}(t) \stackrel{\text { def }}{=}\left\|\bar{u}^{n}\right\|_{\tilde{L}_{t}^{\infty}\left(\dot{B}_{2,1}^{1 / 2}\right)}+\left\|\bar{u}^{n}\right\|_{L_{t}^{1}\left(\dot{B}_{2,1}^{5 / 2}\right)}+\|\nabla \bar{\Pi}\|_{L_{t}^{1}\left(B_{2,1}^{1 / 2}\right)}$. We deduce from (4.15) that

$$
\begin{aligned}
& \left\|H_{n}\right\|_{L_{t}^{1}\left(\dot{B}_{2,1}^{1 / 2}\right)}+\left(1+2^{2 m}\left\|\dot{S}_{m} a\right\|_{L_{t}^{\infty}\left(L^{2}\right)}\right)\left\|\operatorname{div} H_{n}\right\|_{L_{t}^{1}\left(\dot{H}^{-1}\right)} \\
& \leqq \eta Z^{n}(t)+C\left(Z^{n}(t)\right)^{2}+C_{\eta} t\left(1+2^{8 m}\left\|a_{0}\right\|_{L^{2}}^{4}\right)\left(\left\|u_{0}\right\|_{\dot{B}_{2,1}^{1 / 2}}+Z^{n}(t)\right)\left(Z^{n}(t)\right)^{4} \\
& +C \int_{0}^{t} Z^{n}(\tau)\left\|u_{L}^{n}\right\|_{\dot{B}_{2,1}^{5 / 2}} \mathrm{~d} \tau+C\left(1+\left\|a^{n}\right\|_{L_{t}^{\infty}\left(\dot{B}_{2,1}^{3 / 2}\right)}+\left\|u_{0}\right\|_{\left.\dot{B}_{2,1}^{1 / 2}\right)}\left\|u_{L}^{n}\right\|_{L_{t}^{1}\left(\dot{B}_{2,1}^{5 / 2}\right)}\right. \\
& +C t\left(1+2^{8 m}\left\|a_{0}\right\|_{L^{2}}^{4}\right)\left\|u_{0}\right\|_{\dot{B}_{2,1}^{1 / 2}}\left(\left\|u_{0}\right\|_{\dot{B}_{2,1}^{1 / 2}}^{4}+\left\|a^{n}\right\|_{L_{t}^{\infty}\left(\dot{B}_{2,1}^{3 / 2}\right)}^{4}\right) \text {. }
\end{aligned}
$$

Applying (3.12) to the first equation of (4.4) together with (4.14), (4.15) and

$$
\mathrm{e}^{x}-1 \leqq x e^{x} \quad \text { for } x \geqq 0,
$$


we deduce that

$$
\begin{aligned}
& \left\|a^{n}-\dot{S}_{m} a^{n}\right\|_{\widetilde{L}_{t}^{\infty}\left(\dot{B}_{2,1}^{3 / 2}\right)} \leqq\left\|a^{n}-S_{m} a^{n}\right\|_{\widetilde{L}_{t}^{\infty}\left(B_{2,1}^{3 / 2}\right)} \\
& \quad \leqq \sum_{q \geqq m} 2^{3 q / 2}\left\|\Delta_{q} a_{0}\right\|_{L^{2}}+\left\|a_{0}\right\|_{B_{2,1}^{3 / 2}}\left(\exp \left\{C\left(Z^{n}(t)+\left\|u_{L}^{n}\right\|_{L_{t}^{1}\left(\dot{B}_{2,1}^{5 / 2}\right)}\right)\right\}-1\right) \\
& \quad \leqq c_{0} \underline{b}+C\left\|a_{0}\right\|_{B_{2,1}^{3 / 2}}\left(Z^{n}(t)+\left\|u_{L}^{n}\right\|_{L_{t}^{1}\left(\dot{B}_{2,1}^{5 / 2}\right)}\right) \exp \left\{C\left(Z^{n}(t)+\left\|u_{L}^{n}\right\|_{L_{t}^{1}\left(\dot{B}_{2,1}^{5 / 2}\right)}\right)\right\},
\end{aligned}
$$

which along with (4.3) implies

$$
\begin{aligned}
& \left\|a^{n}-S_{m} a^{n}\right\|_{\widetilde{L}_{t}^{\infty}\left(B_{2,1}^{3 / 2}\right)} \leqq c_{0} \underline{b}+C_{1}\left\|a_{0}\right\|_{B_{2,1}^{3 / 2}} \exp \left(C_{1}\left\|u_{0}\right\|_{\dot{B}_{2,1}^{1 / 2}}\right) Z^{n}(t) \mathrm{e}^{C_{1} Z^{n}(t)} \\
& \quad+C_{1}\left\|a_{0}\right\|_{B_{2,1}^{3 / 2}} \exp \left(C_{1}\left\|u_{0}\right\|_{\left.\dot{B}_{2,1}^{1 / 2}\right)} \mathrm{e}^{C_{1} Z^{n}(t)} \sum_{q \in \mathbb{Z}} 2^{q / 2}\left(1-\mathrm{e}^{-c_{1} t 2^{2 q}}\right)\left\|\dot{\Delta}_{q} u_{0}\right\|_{L^{2}}\right.
\end{aligned}
$$

Then thanks to (4.15), (4.16), (4.19) and taking $\eta>0$ sufficiently small in (4.19), we get by applying Proposition 6 to (4.4) together with (3.9), (4.14) and the fact

$$
1+x \leqq \mathrm{e}^{x} \text { for } x \geqq 0
$$

that

$$
\begin{aligned}
& Z^{n}(t) \leqq C \int_{0}^{t}\left(N_{m}+\left\|u_{L}^{n}\right\|_{\dot{B}_{2,1}^{5 / 2}}\right) Z^{n}(\tau) \mathrm{d} \tau
\end{aligned}
$$

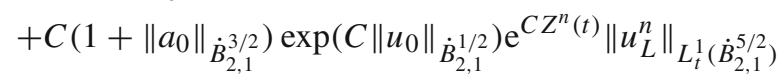

$$
\begin{aligned}
& +C t\left(1+2^{8 m}\left\|a_{0}\right\|_{L^{2}}^{4}\right)\left\|u_{0}\right\|_{\dot{B}_{2,1}^{1 / 2}}\left(\left\|u_{0}\right\|_{\dot{B}_{2,1}^{1 / 2}}^{4}+\left\|a_{0}\right\|_{\dot{B}_{2,1}^{3 / 2}}^{4} \exp \left\{C\left\|u_{0}\right\|_{\dot{B}_{2,1}^{1 / 2}}\right\} \mathrm{e}^{C Z^{n}(t)}\right) \\
& +C t\left(1+2^{8 m}\left\|a_{0}\right\|_{L^{2}}^{4}\right)\left(\left\|u_{0}\right\|_{\dot{B}_{2,1}^{1 / 2}}+Z^{n}(t)\right)\left(Z^{n}(t)\right)^{4}+C\left(Z^{n}(t)\right)^{2},
\end{aligned}
$$

under the assumption

$$
\left\|a^{n}-S_{m} a^{n}\right\|_{\widetilde{L}_{t}^{\infty}\left(B_{2,1}^{3 / 2}\right)} \leqq 2 c_{0} \underline{b} .
$$

Applying (4.3) and Gronwall's inequality yields

$$
\begin{aligned}
& Z^{n}(t) \leqq C_{2} \exp \left(C_{2}\left(t N_{m}+\left\|u_{0}\right\|_{\dot{B}_{2,1}^{1 / 2}}\right)\right) \\
& \times\left\{\left[1+t 2^{8 m}\left\|a_{0}\right\|_{L^{2}}^{4}\left(\left\|u_{0}\right\|_{\dot{B}_{2,1}^{1 / 2}}+Z^{n}(t)\right)\left(Z^{n}(t)\right)^{2}\right]\left(Z^{n}(t)\right)^{2}\right. \\
& +C_{2} t 2^{8 m}\left\|a_{0}\right\|_{L^{2}}^{4}\left\|u_{0}\right\|_{\dot{B}_{2,1}^{1 / 2}}\left(\left\|u_{0}\right\|_{\dot{B}_{2,1}^{1 / 2}}^{4}+\left\|a_{0}\right\|_{\dot{B}_{2,1}^{3 / 2}}^{4} \exp \left(C_{2}\left\|u_{0}\right\|_{\dot{B}_{2,1}^{1 / 2}}\right) \mathrm{e}^{C_{2} Z^{n}(t)}\right) \\
& +\left(1+\left\|a_{0}\right\|_{\dot{B}_{2,1}^{3 / 2}}\right) \exp \left(C_{2}\left\|u_{0}\right\|_{\left.\dot{B}_{2,1}^{1 / 2}\right)} \mathrm{e}^{C_{2} Z^{n}(t)} \sum_{q \in \mathbb{Z}} 2^{q / 2}\left(1-\mathrm{e}^{-c_{1} t 2^{2 q}}\right)\left\|\dot{\Delta}_{q} u_{0}\right\|_{L^{2}}\right\},
\end{aligned}
$$

where we assume the constant $C_{2}>C_{1}>1$. Taking $0<T_{1}\left(\varepsilon_{0}\right) \leqq\left(C_{2} N_{m}\right)^{-1}$ so small that 
$e C_{2}\left(1+\left\|a_{0}\right\|_{\dot{B}_{2,1}^{3 / 2}}\right) \exp \left(2 C_{2}\left\|u_{0}\right\|_{\dot{B}_{2,1}^{1 / 2}}\right) \sum_{q \in \mathbb{Z}} 2^{q / 2}\left(1-\mathrm{e}^{-c_{1} T_{1} 2^{2 q}}\right)\left\|\dot{\Delta}_{q} u_{0}\right\|_{L^{2}} \leqq \varepsilon_{0}$,

for some sufficiently small positive constant $\varepsilon_{0}$. Then it follows from (4.22), (4.20) and (4.21) that

$$
\begin{aligned}
& Z^{n}(t) \leqq \varepsilon_{0} \mathrm{e}^{C_{2} Z^{n}(t)} \\
& \quad+e C_{2} \exp \left(C_{2}\left\|u_{0}\right\|_{B_{2,1}^{1 / 2}}\right)\left\{\left[1+t 2^{8 m}\left\|a_{0}\right\|_{L^{2}}^{4}\left(\left\|u_{0}\right\|_{\dot{B}_{2,1}^{1 / 2}}+Z^{n}(t)\right)\left(Z^{n}(t)\right)^{2}\right]\left(Z^{n}(t)\right)^{2}\right. \\
& \quad+t 2^{8 m}\left\|a_{0}\right\|_{L^{2}}^{4}\left\|u_{0}\right\|_{\dot{B}_{2,1}^{1 / 2}}\left(\left\|u_{0}\right\|_{\dot{B}_{2,1}^{1 / 2}}^{4}+\left\|a_{0}\right\|_{\dot{B}_{2,1}^{3 / 2}}^{4} \exp \left(C_{2}\left\|u_{0}\right\|_{\left.\left.\left.\dot{B}_{2,1}^{1 / 2}\right) \mathrm{e}^{C_{2} Z^{n}(t)}\right)\right\},}\right.\right.
\end{aligned}
$$

provided that

$$
\begin{aligned}
& C_{1}\left\|a_{0}\right\|_{B_{2,1}^{3 / 2}} \exp \left(C_{1}\left\|u_{0}\right\|_{\dot{B}_{2,1}^{1 / 2}}\right) \mathrm{e}^{C_{1} Z^{n}(t)} \\
& \times\left(\sum_{q \in \mathbb{Z}} 2^{q / 2}\left(1-\mathrm{e}^{-c_{1} T_{1} 2^{2 q}}\right)\left\|\dot{\Delta}_{q} u_{0}\right\|_{L^{2}}+Z^{n}(t)\right) \leqq c_{0} \underline{b} .
\end{aligned}
$$

Let $T_{*}^{n}\left(\varepsilon_{0}\right) \stackrel{\text { def }}{=} \sup \left\{t \in\left[0, T_{1}\left(\varepsilon_{0}\right)\right] \mid Z^{n}(t) \leqq 4 e \varepsilon_{0}\right\}$. Without loss of generality, we may assume that $\varepsilon_{0}$ is so small such that

$$
4 e C_{2} \varepsilon_{0} \leqq 1 \text { and } e \varepsilon_{0}+4 e^{2} C_{1} \varepsilon_{0}\left\|a_{0}\right\|_{B_{2,1}^{3 / 2}} \exp \left(C_{1}\left\|u_{0}\right\|_{\dot{B}_{2,1}^{1 / 2}}\right) \leqq c_{0} \underline{b} .
$$

Then for $t \leqq T_{*}^{n}\left(\varepsilon_{0}\right)$, we get from (4.23) that (4.24) holds and then

$$
\begin{aligned}
& Z^{n}(t) \leqq e C_{2} \exp \left(C_{2}\left\|u_{0}\right\|_{\dot{B}_{2,1}^{1 / 2}}\right) \\
& \quad \times\left\{\left(1+t\left(4 e \varepsilon_{0}\right)^{2} 2^{8 m}\left\|a_{0}\right\|_{L^{2}}^{4}\left(\left\|u_{0}\right\|_{\dot{B}_{2,1}^{1 / 2}}+4 e \varepsilon_{0}\right)\right) 4 e \varepsilon_{0} Z^{n}(t)\right. \\
& \quad+t 2^{8 m}\left\|a_{0}\right\|_{L^{2}}^{4}\left\|u_{0}\right\|_{\dot{B}_{2,1}^{1 / 2}}\left(\left\|u_{0}\right\|_{\dot{B}_{2,1}^{1 / 2}}^{4}+e\left\|a_{0}\right\|_{\dot{B}_{2,1}^{3 / 2}}^{4} \exp \left(C_{2}\left\|u_{0}\right\|_{\left.\left.\left.\dot{B}_{2,1}^{1 / 2}\right)\right)\right\}+e \varepsilon_{0}}\right.\right. \\
& \quad \stackrel{\text { def }}{=} e C_{2} \exp \left(C_{2}\left\|u_{0}\right\|_{\dot{B}_{2,1}^{\frac{1}{2}}}\right)\left\{\left(1+t \mathcal{A}_{1}\right) 4 e \varepsilon_{0} Z^{n}(t)+t \mathcal{A}_{2}\right\}+e \varepsilon_{0},
\end{aligned}
$$

where

$$
\begin{aligned}
& \mathcal{A}_{1}=\left(4 e \varepsilon_{0}\right)^{2} 2^{8 m}\left\|a_{0}\right\|_{L^{2}}^{4}\left(\left\|u_{0}\right\|_{\dot{B}_{2,1}^{1 / 2}}+4 e \varepsilon_{0}\right), \text { and } \\
& \mathcal{A}_{2}=2^{8 m}\left\|a_{0}\right\|_{L^{2}}^{4}\left\|u_{0}\right\|_{\dot{B}_{2,1}^{1 / 2}}\left(\left\|u_{0}\right\|_{\dot{B}_{2,1}^{1 / 2}}^{4}+e\left\|a_{0}\right\|_{\dot{B}_{2,1}^{3 / 2}}^{4} \exp \left(C_{2}\left\|u_{0}\right\|_{\dot{B}_{2,1}^{1 / 2}}\right)\right) .
\end{aligned}
$$

Taking $\varepsilon_{0}$ so small that

$$
\begin{aligned}
& 4 e^{2} C_{2} \exp \left(C_{2}\left\|u_{0}\right\|_{\left.\dot{B}_{2,1}^{1 / 2}\right) \varepsilon_{0}} \leqq \frac{1}{8}\right. \text { and } \\
& T_{2}\left(\varepsilon_{0}\right)=\min \left(T_{1}\left(\varepsilon_{0}\right), \frac{1}{\mathcal{A}_{1}}, \frac{e \varepsilon_{0}}{e C_{2} \exp \left(C_{2}\left\|u_{0}\right\|_{\dot{B}_{2,1}^{\frac{1}{2}}}\right) \mathcal{A}_{2}}\right),
\end{aligned}
$$


we conclude that

$$
Z^{n}(t) \leqq \frac{8}{3} e \varepsilon_{0} \quad \text { for } t \leqq T_{2}\left(\varepsilon_{0}\right)
$$

This, together with (4.3) and (4.15), ensures

$$
\left\{a^{n}, u^{n}, \nabla \Pi^{n}\right\}_{n \in \mathbb{N}} \text { is uniformly bounded in } E_{T_{2}\left(\varepsilon_{0}\right)} \text {. }
$$

On the other hand, if $\left\|u_{0}\right\|_{\dot{B}_{2,1}^{1 / 2}}$ is sufficiently small, we apply Remark 8 to the system (1.2) with initial data $\left(a_{0}^{n}, u_{0}^{n}\right)$, and using (4.15) to get

$$
\begin{aligned}
& X^{n}(t) \stackrel{\text { def }}{=}\left\|u^{n}\right\|_{\widetilde{L}_{t}^{\infty}\left(\dot{B}_{2,1}^{1 / 2}\right)}+\left\|u^{n}\right\|_{L_{t}^{1}\left(\dot{B}_{2,1}^{5 / 2}\right)}+\left\|\nabla \Pi^{n}\right\|_{L_{t}^{1}\left(\dot{B}_{2,1}^{1 / 2}\right)} \\
& \quad \leqq C\left(\left\|u_{0}\right\|_{\dot{B}_{2,1}^{1 / 2}}+\int_{0}^{t}\left(\left\|\nabla u^{n}\right\|_{L^{\infty}}+W_{1}^{n}(\tau)+2^{8 m}\left\|a_{0}\right\|_{L^{2}}^{4}\left(X^{n}(\tau)\right)^{4}\right)\left\|u^{n}\right\|_{\dot{B}_{2,1}^{1 / 2}} \mathrm{~d} \tau\right)
\end{aligned}
$$

with

$$
\begin{gathered}
W_{1}^{n}(t) \stackrel{\text { def }}{=} 2^{8 m}\|a\|_{L_{t}^{\infty}\left(L^{2}\right)}^{4}\left(1+\|a\|_{L_{t}^{\infty}\left(L^{\infty}\right)}^{4}\right)+2^{2 m}\|a\|_{L_{t}^{\infty}\left(L^{\infty}\right)}^{2} \\
\leqq 2^{8 m}\left\|a_{0}\right\|_{L^{2}}^{4}\left(1+\left\|a_{0}\right\|_{L^{\infty}}^{4}\right)+2^{2 m}\left\|a_{0}\right\|_{L^{\infty}}^{2} \stackrel{\text { def }}{=} \bar{N}_{m}
\end{gathered}
$$

under the assumption (4.21).

Remark 5 applied to the first equation of (1.2) together with (4.14) gives rise to

$$
\begin{aligned}
\left\|a^{n}-S_{m} a^{n}\right\|_{\widetilde{L}_{t}^{\infty}\left(B_{2,1}^{3 / 2}\right)} & \leqq c_{0} \underline{b}+\left\|a_{0}\right\|_{B_{2,1}^{3 / 2}\left(\exp \left\{C_{3} X^{n}(t)\right\}-1\right)} \\
& \leqq c_{0} \underline{b}+C_{3} X^{n}(t)\left\|a_{0}\right\|_{B_{2,1}^{3 / 2}} \exp \left\{C_{3} X^{n}(t)\right\}
\end{aligned}
$$

Therefore, we get from (4.27) that

$$
X^{n}(t) \leqq C_{4}\left\|u_{0}\right\|_{\dot{B}_{2,1}^{\frac{1}{2}}} \exp \left\{C_{4}\left(\bar{N}_{m} t+X^{n}(t)+t 2^{8 m}\left\|a_{0}\right\|_{L^{2}}^{4}\left(X^{n}(t)\right)^{4}\right)\right\}
$$

provided that

$$
C_{3} X^{n}(t)\left\|a_{0}\right\|_{B_{2,1}^{3 / 2}} \exp \left\{C_{3} X^{n}(t)\right\} \leqq c_{0} \underline{b}
$$

where, without loss of generality, we assume the constant $C_{4}>C_{3}>1$. Taking $\left\|u_{0}\right\|_{\dot{B}_{2,1}^{1 / 2}}$ so small that

$$
8 C_{4}^{2}\left(1+2^{8 m}\left\|a_{0}\right\|_{L^{2}}^{4}+\left\|a_{0}\right\|_{\left.B_{2,1}^{3 / 2}\right)}\left\|u_{0}\right\|_{\dot{B}_{2,1}^{1 / 2}} \exp \left\{4 C_{4} \bar{N}_{m}\right\} \leqq \min \left\{1, c_{0} \underline{b}\right\},\right.
$$

we claim that there exists a positive time $T>1$ such that

$$
X^{n}(T) \leqq 2 C_{4}\left\|u_{0}\right\|_{\dot{B}_{2,1}^{1 / 2}} \exp \left\{2 C_{4} \bar{N}_{m}\right\} \quad\left(\leqq \frac{\min \left\{1, c_{0} \underline{b}\right\}}{4 C_{4}}\right) .
$$


Indeed, we define

$$
T^{*} \stackrel{\text { def }}{=} \sup \left\{T>0: \quad X^{n}(T) \leqq 2 C_{4}\left\|u_{0}\right\|_{\dot{B}_{2,1}^{1 / 2}} \exp \left\{2 C_{4} \bar{N}_{m}\right\}\right\},
$$

hence for $\forall T<T^{*},(4.29)$ holds.

If $T^{*} \leqq \frac{3}{2}$, then we get from (4.30) that for $\forall T<T^{*}$

$$
C_{4} X^{n}(T)<\frac{1}{4} \text { and } C_{4} T 2^{8 m}\left\|a_{0}\right\|_{L^{2}}^{4}\left(X^{n}(T)\right)^{4}<\frac{T}{4}\left(X^{n}(T)\right)^{3} \leqq \frac{1}{4^{3}},
$$

which together with (4.28) leads to

$$
X^{n}(T)<C_{4}\left\|u_{0}\right\|_{\dot{B}_{2,1}^{1 / 2}} \exp \left\{C_{4} \bar{N}_{m} T+\frac{1}{4}+\frac{1}{4^{3}}\right\} \leqq C_{4}\left\|u_{0}\right\|_{\dot{B}_{2,1}^{1 / 2}} \exp \left\{2 C_{4} \bar{N}_{m}\right\} .
$$

This contradicts (4.31), and thus $T^{*}>\frac{3}{2}$.

On the other hand, Proposition 1 applied to the transport equation of (1.2) yields

$$
\left\|a^{n}\right\|_{\widetilde{L}_{T}^{\infty}\left(\dot{B}_{2,1}^{3 / 2}\right)} \leqq C_{5}\left\|a_{0}\right\|_{\dot{B}_{2,1}^{3 / 2}} \exp \left\{C_{5} X^{n}(T)\right\} .
$$

Therefore, $\left(a^{n}, u^{n}, \nabla \Pi^{n}\right)$ is uniformly bounded in $E_{T}$ with some $T>1$ and (4.1) holds.

Step 3: Convergence

With (4.25), it follows from Step 3 in the proof of Theorem 5.1 in [9] that up to a subsequence $\left\{\left(a^{n}, u^{n}, \nabla \Pi^{n}\right)\right\}_{n \in \mathbb{N}}$ converges to some limit $(a, u, \nabla \Pi)$ on $[0, T]$, which solves (1.2) and satisfies (4.1). Moreover, there holds

$$
\left\|a-S_{m} a\right\|_{\widetilde{L}_{T}^{\infty}\left(B_{2,1}^{3 / 2}\right)} \leqq 2 c_{0} \underline{b}
$$

with the constant $c_{0}$ being sufficiently small.

\subsection{Uniqueness Part of Theorem 2}

Let $\left(a^{i}, u^{i}, \nabla \Pi^{i}\right)$ (with $\left.i=1,2\right)$ be two solutions of the system (1.2), which satisfy (4.32) and

$$
\left(a^{i}, u^{i}, \nabla \Pi^{i}\right) \in \mathcal{C}_{b}\left([0, T] ; B_{2,1}^{3 / 2}\right) \times\left(L_{T}^{1}\left(\dot{B}_{2,1}^{5 / 2}\right) \cap \mathcal{C}_{b}\left([0, T] ; B_{2,1}^{1 / 2}\right)\right) \times L_{T}^{1}\left(B_{2,1}^{1 / 2}\right) .
$$

We define

$$
(\delta a, \delta u, \nabla \delta \Pi) \stackrel{\text { def }}{=}\left(a^{2}-a^{1}, u^{2}-u^{1}, \nabla \Pi^{2}-\nabla \Pi^{1}\right),
$$

so that $(\delta a, \delta u, \nabla \delta \Pi)$ solves

$$
\left\{\begin{array}{l}
\partial_{t} \delta a+u^{2} \cdot \nabla \delta a=-\delta u \cdot \nabla a^{1} \\
\partial_{t} \delta u+u^{2} \cdot \nabla \delta u-\left(1+a^{2}\right)(\Delta \delta u-\nabla \delta \Pi)=-\delta u \cdot \nabla u^{1}+\delta a\left(\Delta u^{1}-\nabla \Pi^{1}\right) \\
\operatorname{div} \delta u=0 \\
\left.(\delta a, \delta u)\right|_{t=0}=(0,0)
\end{array}\right.
$$


and satisfies

$$
\begin{aligned}
(\delta a, \delta u, \nabla \delta \Pi) & \in \mathcal{C}_{b}\left([0, T] ; B_{2,1}^{3 / 2}\right) \times\left(L_{T}^{1}\left(B_{2,1}^{3 / 2}\right)\right. \\
& \left.\cap \mathcal{C}_{b}\left([0, T] ; B_{2,1}^{-1 / 2}\right)\right) \times L_{T}^{1}\left(B_{2,1}^{-1 / 2}\right) .
\end{aligned}
$$

We first write the momentum equation of (4.34) as

$$
\partial_{t} \delta u+u^{2} \cdot \nabla \delta u-\left(1+S_{m} a^{2}\right)(\Delta \delta u-\nabla \delta \Pi)=H
$$

with $H=\left(a^{2}-S_{m} a^{2}\right)(\Delta \delta u-\nabla \delta \Pi)-\delta u \cdot \nabla u^{1}+\delta a\left(\Delta u^{1}-\nabla \Pi^{1}\right)$. Proposition 5 applied to (4.35) yields that for $\forall 0<t \leqq T$

$$
\begin{aligned}
& \|\delta u\|_{\widetilde{L}_{t}^{\infty}\left(B_{2,1}^{-1 / 2}\right)}+\|\delta u\|_{L_{t}^{1}\left(B_{2,1}^{3 / 2}\right)} \\
& \leq C\left\{\int_{0}^{t}\left\|\nabla u^{2}(\tau)\right\|_{B_{2,1}^{3 / 2}\|\delta u\|_{B_{2,1}^{-1 / 2}} \mathrm{~d} \tau+\|\delta u\|_{L_{t}^{1}\left(L^{2}\right)}+\|H\|_{L_{t}^{1}\left(B_{2,1}^{-1 / 2}\right)}}\right. \\
& \left.\quad+\left\|S_{m} a^{2}\right\|_{L_{t}^{\infty}\left(H^{2}\right)}\left(\|\nabla \delta \Pi\|_{L_{t}^{1}\left(H^{-1}\right)}+\|\nabla \delta u\|_{L_{t}^{1}\left(L^{2}\right)}\right)\right\}
\end{aligned}
$$

On the other hand, applying div to the momentum equation of (4.34) yields

$$
\operatorname{div}\left[\left(1+S_{m} a^{2}\right) \nabla \delta \Pi\right]=\operatorname{div} G
$$

with

$$
\begin{aligned}
G & =\left(S_{m} a^{2}-a^{2}\right) \nabla \delta \Pi+a^{2} \Delta \delta u-\delta u \cdot \nabla u^{1}-u^{2} \cdot \nabla \delta u+\delta a\left(\Delta u^{1}-\nabla \Pi^{1}\right) \\
& \stackrel{\text { def }}{=}\left(S_{m} a^{2}-a^{2}\right) \nabla \delta \Pi+a^{2} \Delta \delta u+G_{1} .
\end{aligned}
$$

Then applying Proposition 3 gives

$$
\begin{aligned}
& \|\nabla \delta \Pi\|_{L_{t}^{1}\left(B_{2,1}^{-1 / 2}\right)} \lesssim\|G\|_{L_{t}^{1}\left(B_{2,1}^{-1 / 2}\right)}+\left\|S_{m} a^{2}\right\|_{L_{t}^{\infty}\left(H^{2}\right)}\|\nabla \delta \Pi\|_{L_{t}^{1}\left(H^{-1}\right)} \\
& \lesssim\left\|S_{m} a^{2}\right\|_{L_{t}^{\infty}\left(H^{2}\right)}\|\nabla \delta \Pi\|_{L_{t}^{1}\left(H^{-1}\right)}+\left\|a^{2}-S_{m} a^{2}\right\|_{L_{t}^{\infty}\left(B_{2,1}^{3 / 2}\right)}\|\nabla \delta \Pi\|_{L_{t}^{1}\left(B_{2,1}^{-1 / 2}\right)} \\
& \quad+\left\|a^{2}\right\|_{L_{t}^{\infty}\left(B_{2,1}^{3 / 2}\right)}\|\Delta \delta u\|_{L_{t}^{1}\left(B_{2,1}^{-1 / 2}\right)}+\left\|G_{1}\right\|_{L_{t}^{1}\left(B_{2,1}^{-1 / 2}\right)} \cdot
\end{aligned}
$$

Due to (4.32), one has

$$
\left(1+\left\|a^{2}\right\|_{L_{T}^{\infty}\left(B_{2,1}^{3 / 2}\right)}\right)\left\|a^{2}-S_{m} a^{2}\right\|_{L_{T}^{\infty}\left(B_{2,1}^{3 / 2}\right)}<C c_{0} \underline{b},
$$

which gives rise to

$$
\begin{aligned}
\|\nabla \delta \Pi\|_{L_{t}^{1}\left(B_{2,1}^{-1 / 2}\right)} \lesssim & \left\|S_{m} a^{2}\right\|_{L_{t}^{\infty}\left(H^{2}\right)}\|\nabla \delta \Pi\|_{L_{T}^{1}\left(H^{-1}\right)} \\
& +\left\|a^{2}\right\|_{L_{t}^{\infty}\left(B_{2,1}^{3 / 2}\right)}\|\Delta \delta u\|_{L_{t}^{1}\left(B_{2,1}^{-1 / 2}\right)}+\left\|G_{1}\right\|_{L_{t}^{1}\left(B_{2,1}^{-1 / 2}\right)} .
\end{aligned}
$$


From this, we infer

$$
\begin{aligned}
& \left\|\left(a^{2}-S_{m} a^{2}\right)(\Delta \delta u-\nabla \delta \Pi)\right\|_{L_{t}^{1}\left(B_{2,1}^{-1 / 2}\right)} \\
& \lesssim\left\|a^{2}-S_{m} a^{2}\right\|_{L_{t}^{\infty}\left(B_{2,1}^{3 / 2}\right)}\left(\|\Delta \delta u\|_{L_{t}^{1}\left(B_{2,1}^{-1 / 2}\right)}+\|\nabla \delta \Pi\|_{L_{t}^{1}\left(B_{2,1}^{-1 / 2}\right)}\right) \\
& \lesssim\left\|a^{2}-S_{m} a^{2}\right\|_{L_{t}^{\infty}\left(B_{2,1}^{3 / 2}\right)}\left(1+\left\|a^{2}\right\|_{L_{t}^{\infty}\left(B_{2,1}^{3 / 2}\right)}\|\Delta \delta u\|_{L_{t}^{1}\left(B_{2,1}^{-1 / 2}\right)}\right. \\
& \quad+\left\|a^{2}-S_{m} a^{2}\right\|_{L_{t}^{\infty}\left(B_{2,1}^{3 / 2}\right)}\left(\left\|S_{m} a^{2}\right\|_{L_{t}^{\infty}\left(H^{2}\right)}\|\nabla \delta \Pi\|_{L_{T}^{1}\left(H^{-1}\right)}+\left\|G_{1}\right\|_{L_{t}^{1}\left(B_{2,1}^{-1 / 2}\right)} .\right.
\end{aligned}
$$

Therefore, plugging (4.39) into (4.36) and taking the constant $c_{0}$ small enough in (4.37), we conclude that

$$
\begin{aligned}
& \|\delta u\|_{\widetilde{L}_{t}^{\infty}\left(B_{2,1}^{-1 / 2}\right)}+\|\delta u\|_{L_{t}^{1}\left(B_{2,1}^{3 / 2}\right)} \lesssim \int_{0}^{t}\left\|\nabla u^{2}(\tau)\right\|_{B_{2,1}^{3 / 2}}\|\delta u\|_{B_{2,1}^{-1 / 2}} \mathrm{~d} \tau+\|\delta u\|_{L_{t}^{1}\left(L^{2}\right)} \\
& +\left\|S_{m} a^{2}\right\|_{L_{t}^{\infty}\left(H^{2}\right)}\left(\|\nabla \delta \Pi\|_{L_{t}^{1}\left(H^{-1}\right)}+\|\delta u\|_{L_{t}^{1}\left(H^{1}\right)}\right)+\left\|\delta a\left(\Delta u^{1}-\nabla \Pi^{1}\right)\right\|_{L_{t}^{1}\left(B_{2,1}^{-1 / 2}\right)} \\
& +\left\|\delta u \cdot \nabla u^{1}\right\|_{L_{t}^{1}\left(B_{2,1}^{-1 / 2}\right)}+\left\|u^{2} \cdot \nabla \delta u\right\|_{L_{t}^{1}\left(B_{2,1}^{-1 / 2}\right)}
\end{aligned}
$$

While applying Proposition 1 to the first equation in (4.34) yields that for all $t \leqq T$

$$
\|\delta a\|_{\widetilde{L}_{t}^{\infty}\left(B_{2,1}^{1 / 2}\right)} \lesssim \exp \left\{C\left\|\nabla u^{2}\right\|_{L_{t}^{1}\left(B_{2,1}^{3 / 2}\right)}\right\}\left\|a^{1}\right\|_{L_{t}^{\infty}\left(B_{2,1}^{3 / 2}\right)}\|\delta u\|_{L_{t}^{1}\left(B_{2,1}^{3 / 2}\right)}
$$

which along with the product law in Besov spaces ensures

$$
\begin{aligned}
& \left\|\delta a\left(\Delta u^{1}-\nabla \Pi^{1}\right)\right\|_{L_{t}^{1}\left(B_{2,1}^{-1 / 2}\right)} \lesssim \int_{0}^{t}\|\delta a\|_{B_{2,1}^{1 / 2}}\left\|\Delta u^{1}-\nabla \Pi^{1}\right\|_{B_{2,1}^{1 / 2}} \mathrm{~d} \tau \\
& \lesssim \int_{0}^{t}\left\|a^{1}\right\|_{L_{\tau}^{\infty}\left(B_{2,1}^{3 / 2}\right)}\|\delta u\|_{L_{\tau}^{1}\left(B_{2,1}^{3 / 2}\right)} \\
& \quad \times\left(\left\|\Delta u^{1}\right\|_{B_{2,1}^{1 / 2}}+\left\|\nabla \Pi^{1}\right\|_{\left.B_{2,1}^{1 / 2}\right)} \exp \left\{C\left\|\nabla u^{2}\right\|_{L_{\tau}^{1}\left(B_{2,1}^{3 / 2}\right)}\right\} \mathrm{d} \tau .\right.
\end{aligned}
$$

Notice that $\|\delta u\|_{B_{2,1}^{1 / 2}} \lesssim\|\delta u\|_{B_{2,1}^{-1 / 2}}^{1 / 2}\|\delta u\|_{B_{2,1}^{3 / 2}}^{1 / 2}$, applying Young's inequality gives for every $\varepsilon>0$

$$
\begin{aligned}
& \left\|\delta u \cdot \nabla u^{1}\right\|_{L_{t}^{1}\left(B_{2,1}^{-1 / 2}\right)}+\left\|u^{2} \cdot \nabla \delta u\right\|_{L_{t}^{1}\left(B_{2,1}^{-1 / 2}\right)}
\end{aligned}
$$

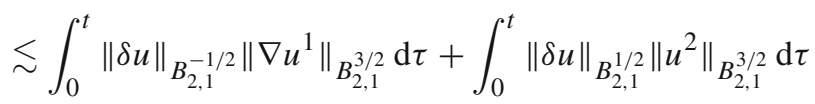

$$
\begin{aligned}
& \leqq \varepsilon\|\delta u\|_{L_{t}^{1}\left(B_{2,1}^{3 / 2}\right)}+C_{\varepsilon} \int_{0}^{t}\|\delta u\|_{B_{2,1}^{-1 / 2}}\left(\left\|\nabla u^{1}\right\|_{B_{2,1}^{3 / 2}}+\left\|u^{2}\right\|_{B_{2,1}^{3 / 2}}^{2}\right) \mathrm{d} \tau \text {. }
\end{aligned}
$$

Plugging (4.42) and (4.43) into (4.40) and taking $\varepsilon>0$ sufficiently small, we obtain 


$$
\begin{aligned}
& \|\delta u\|_{\widetilde{L}_{t}^{\infty}\left(B_{2,1}^{-1 / 2}\right)}+\|\delta u\|_{L_{t}^{1}\left(B_{2,1}^{3 / 2}\right)} \\
& \lesssim \int_{0}^{t}\|\delta u\|_{B_{2,1}^{-1 / 2}}\left(\left\|\nabla u^{1}\right\|_{B_{2,1}^{3 / 2}}+\left\|\nabla u^{2}(\tau)\right\|_{B_{2,1}^{3 / 2}}+\left\|u^{2}\right\|_{B_{2,1}^{3 / 2}}^{2}\right) \mathrm{d} \tau \\
& \quad+\left\|S_{m} a^{2}\right\|_{L_{t}^{\infty}\left(H^{2}\right)}\left(\|\nabla \delta \Pi\|_{L_{t}^{1}\left(H^{-1}\right)}+\|\delta u\|_{L_{t}^{1}\left(H^{1}\right)}\right)+\|\delta u\|_{L_{t}^{1}\left(L^{2}\right)} \\
& \quad+\int_{0}^{t}\left\|a^{1}\right\|_{L_{\tau}^{\infty}\left(B_{2,1}^{3 / 2}\right)}\|\delta u\|_{L_{\tau}^{1}\left(B_{2,1}^{3 / 2}\right)} \\
& \quad \times\left(\left\|\Delta u^{1}\right\|_{B_{2,1}^{1 / 2}}+\left\|\nabla \Pi^{1}\right\|_{\left.B_{2,1}^{1 / 2}\right)} \exp \left\{C\left\|\nabla u^{2}\right\|_{L_{\tau}^{1}\left(B_{2,1}^{3 / 2}\right)}\right\} \mathrm{d} \tau,\right.
\end{aligned}
$$

which along with $\left\|S_{m} a^{2}\right\|_{L_{t}^{\infty}\left(H^{2}\right)} \lesssim 2^{2 m}\left\|a_{0}\right\|_{L^{2}}$ and (4.33) yields

$$
\begin{aligned}
& \|\delta u\|_{\widetilde{L}_{t}^{\infty}\left(B_{2,1}^{-1 / 2}\right)}+\|\delta u\|_{L_{t}^{1}\left(B_{2,1}^{3 / 2}\right)} \\
& \leqq C \int_{0}^{t}\|\delta u\|_{B_{2,1}^{-1 / 2}}\left(1+\left\|\nabla u^{1}\right\|_{B_{2,1}^{3 / 2}}+\left\|\nabla u^{2}(\tau)\right\|_{B_{2,1}^{3 / 2}}+\left\|u^{2}\right\|_{B_{2,1}^{3 / 2}}^{2}\right) \mathrm{d} \tau \\
& \quad+C\left(\|\nabla \delta \Pi\|_{L_{t}^{1}\left(H^{-1}\right)}+\|\delta u\|_{L_{t}^{1}\left(H^{1}\right)}\right) \\
& \quad+C \int_{0}^{t}\|\delta u\|_{L_{\tau}^{1}\left(B_{2,1}^{3 / 2}\right)}\left(\left\|\Delta u^{1}\right\|_{B_{2,1}^{1 / 2}}+\left\|\nabla \Pi^{1}\right\|_{\left.B_{2,1}^{1 / 2}\right)} \mathrm{d} \tau\right.
\end{aligned}
$$

Let us now turn to estimating $\|\nabla \delta \Pi\|_{H^{-1}}$. Indeed, applying div to the momentum equation of (4.34) again yields

$$
\operatorname{div}\left[\left(1+a^{2}\right) \nabla \delta \Pi\right]=\operatorname{div} \bar{G},
$$

with $\bar{G}=a^{2} \Delta \delta u-\delta u \cdot \nabla u^{1}-u^{2} \cdot \nabla \delta u+\delta a\left(\Delta u^{1}-\nabla \Pi^{1}\right)$. Thanks to Proposition 4, we get

$$
\|\nabla \delta \Pi\|_{H^{-1}} \lesssim\left(1+2^{m}\left\|a^{2}\right\|_{\left.B_{2,1}^{3 / 2}\right)}\|\bar{G}\|_{H^{-1}}\right.
$$

While thanks to the product law in Besov spaces, we have

$$
\begin{aligned}
& \|\bar{G}\|_{H^{-1}} \\
& \quad \lesssim\left\|a^{2} \Delta \delta u\right\|_{H^{-1}}+\left\|\delta u \cdot \nabla u^{1}\right\|_{H^{-1}}+\left\|u^{2} \cdot \nabla \delta u\right\|_{H^{-1}}+\left\|\delta a\left(\Delta u^{1}-\nabla \Pi^{1}\right)\right\|_{H^{-1}} \\
& \quad \lesssim\left\|a^{2}\right\|_{B_{2,1}^{3 / 2}}\|\Delta \delta u\|_{H^{-1}}+\|\delta u\|_{H^{1}} \sum_{k=1}^{2}\left\|u^{k}\right\|_{H^{1 / 2}}+\|\delta a\|_{B_{2,1}^{1 / 2}}\left\|\Delta u^{1}-\nabla \Pi^{1}\right\|_{L^{2}}
\end{aligned}
$$

which leads to

$$
\begin{aligned}
& \|\nabla \delta \Pi\|_{L_{t}^{1}\left(H^{-1}\right)} \lesssim\left(1+2^{m}\left\|a^{2}\right\|_{L_{t}^{\infty}\left(B_{2,1}^{3 / 2}\right)}\right)\left[\left\|a^{2}\right\|_{L_{t}^{\infty}\left(B_{2,1}^{3 / 2}\right)}\|\Delta \delta u\|_{L_{t}^{1}\left(H^{-1}\right)}\right. \\
& \left.\quad+\|\delta u\|_{L_{t}^{1}\left(H^{1}\right)} \sum_{k=1}^{2}\left\|u^{k}\right\|_{L_{t}^{\infty}\left(H^{1 / 2}\right)}+\int_{0}^{t}\|\delta a\|_{B_{2,1}^{1 / 2}}\left(\left\|\Delta u^{1}\right\|_{L^{2}}+\left\|\nabla \Pi^{1}\right\|_{L^{2}}\right) \mathrm{d} \tau\right] .
\end{aligned}
$$


Therefore, with (4.41) and (4.33), we obtain

$$
\begin{aligned}
& \|\nabla \delta \Pi\|_{L_{t}^{1}\left(H^{-1}\right)} \\
& \lesssim\left(1+2^{m}\left\|a^{2}\right\|_{L_{t}^{\infty}\left(B_{2,1}^{3 / 2}\right)}\left(\left(\left\|a^{2}\right\|_{L_{t}^{\infty}\left(B_{2,1}^{3 / 2}\right)}+\sum_{k=1}^{2}\left\|u^{k}\right\|_{L_{t}^{\infty}\left(H^{1 / 2}\right)}\right)\|\delta u\|_{L_{t}^{1}\left(H^{1}\right)}\right.\right. \\
& \quad+\int_{0}^{t}\left\|a^{1}\right\|_{L_{\tau}^{\infty}\left(B_{2,1}^{3 / 2}\right)}\|\delta u\|_{L_{\tau}^{1}\left(B_{2,1}^{3 / 2}\right)} \\
& \quad \times\left(\left\|\Delta u^{1}\right\|_{L^{2}}+\left\|\nabla \Pi^{1}\right\|_{\left.L^{2}\right)} \exp \left\{C\left\|\nabla u^{2}\right\|_{L_{\tau}^{1}\left(B_{2,1}^{3 / 2}\right)}\right\} \mathrm{d} \tau\right) \\
& \lesssim\|\delta u\|_{L_{t}^{1}\left(H^{1}\right)}+\int_{0}^{t}\|\delta u\|_{L_{\tau}^{1}\left(B_{2,1}^{3 / 2}\right)}\left(\left\|\Delta u^{1}\right\|_{L^{2}}+\left\|\nabla \Pi^{1}\right\|_{L^{2}}\right) \mathrm{d} \tau
\end{aligned}
$$

Note that for $\forall \eta>0$

$$
\begin{aligned}
C\|\delta u\|_{L_{t}^{1}\left(H^{1}\right)} & \leqq C \int_{0}^{t}\|\delta u\|_{B_{2,1}^{-1 / 2}}^{1 / 4}\|\delta u\|_{B_{2,1}^{3 / 2}}^{3 / 4} \mathrm{~d} \tau \\
& \leqq \eta\|\delta u\|_{L_{t}^{1}\left(B_{2,1}^{3 / 2}\right)}+C_{\eta} \int_{0}^{t}\|\delta u\|_{B_{2,1}^{-1 / 2}} \mathrm{~d} \tau .
\end{aligned}
$$

Then plugging (4.46) into (4.44) and taking $\eta>0$ small enough, we arrive at

$$
\begin{aligned}
& \|\delta u\|_{\widetilde{L}_{t}^{\infty}\left(B_{2,1}^{-1 / 2}\right)}+\|\delta u\|_{L_{t}^{1}\left(B_{2,1}^{3 / 2}\right)} \\
& \leqq \\
& \quad C \int_{0}^{t}\|\delta u\|_{B_{2,1}^{-1 / 2}}\left(1+\left\|\nabla u^{1}\right\|_{B_{2,1}^{3 / 2}}+\left\|\nabla u^{2}(\tau)\right\|_{B_{2,1}^{3 / 2}}+\left\|u^{2}\right\|_{B_{2,1}^{3 / 2}}^{2}\right) \mathrm{d} \tau \\
& \quad+C \int_{0}^{t}\|\delta u\|_{L_{\tau}^{1}\left(B_{2,1}^{3 / 2}\right)}\left(\left\|\Delta u^{1}\right\|_{B_{2,1}^{1 / 2}}+\left\|\nabla \Pi^{1}\right\|_{\left.B_{2,1}^{1 / 2}\right)} \mathrm{d} \tau .\right.
\end{aligned}
$$

Applying Gronwall's inequality to the above inequality and using (4.33) implies $\delta u(t)=0$, which together with (4.41) and (4.46) implies that $\delta a(t)=\delta \nabla \Pi(t)=0$ for all $t \in[0, T]$.

\subsection{Higher Regularity Part of Theorem 2}

Proof of (1.8). Let $\left(a^{n}, u^{n}, \Pi^{n}\right)$ to be the approximate solutions of (1.2) constructed in Step 2 of Subsection 4.1. Then for $0<\tau<t_{0}<t \leqq T^{*}$, with $T^{*}$ being determined by (4.31), we deduce by a similar proof of (4.10) and (4.12) that

$$
\begin{aligned}
& \left\|u^{n}\right\|_{\widetilde{L}^{\infty}\left([\tau, t] ; \dot{B}_{2,1}^{3 / 2}\right)}+\left\|u^{n}\right\|_{L^{1}\left([\tau, t] ; \dot{B}_{p, 1}^{7 / 2}\right)}+\left\|\nabla \Pi^{n}\right\|_{L^{1}\left([\tau, t] ; \dot{B}_{2,1}^{3 / 2}\right)} \\
& \quad \lesssim\|u(\tau)\|_{\dot{B}_{2,1}^{3 / 2}}+\left\|\left(a^{n}-S_{m} a^{n}\right)\left(\Delta u^{n}-\nabla \Pi^{n}\right)\right\|_{L^{1}\left([\tau, t] ; \dot{B}_{2,1}^{3 / 2}\right)} \\
& \quad+\left\|\left(\dot{T}_{\Delta u^{n}} \dot{S}_{m} a^{n}, \dot{\mathcal{R}}\left(\dot{S}_{m} a^{n}, \Delta u^{n}\right)\right)\right\|_{L^{1}\left([\tau, t] ; \dot{B}_{2,1}^{3 / 2}\right)}+\left\|\dot{T}_{\nabla \dot{S}_{m} a^{n}} \Delta u^{n}\right\|_{L^{1}\left([\tau, t] ; \dot{B}_{2,1}^{1 / 2}\right)} \\
& \quad+\left\|u^{n} \cdot \nabla u^{n}\right\|_{L^{1}\left([\tau, t] ; \dot{B}_{2,1}^{3 / 2}\right)}+\left\|\Pi^{n} \nabla \dot{S}_{m} a^{n}\right\|_{L^{1}\left([\tau, t] ; \dot{B}_{2,1}^{3 / 2}\right)}
\end{aligned}
$$




$$
\begin{aligned}
& +\sum_{q \in \mathbb{Z}}\left(2^{3 q / 2}\left\|\left[\dot{\Delta}_{q}, \dot{S}_{m} a^{n}\right] \nabla \Pi^{n}\right\|_{L^{1}\left([\tau, t] ; L^{2}\right)}+2^{5 q / 2}\left\|\left[\dot{\Delta}_{q}, \dot{S}_{m} a^{n}\right] \nabla u^{n}\right\|_{L^{1}\left([\tau, t] ; L^{2}\right)}\right) \\
& +\left\|\nabla \dot{S}_{m} a^{n}\right\|_{L^{\infty}\left([\tau, t] ; L^{\infty}\right)}\left\|\nabla u^{n}\right\|_{L^{1}\left([\tau, t] ; \dot{B}_{2,1}^{3 / 2}\right)}+\left\|\nabla \dot{S}_{m} a^{n} \cdot \nabla u^{n}\right\|_{L^{1}\left([\tau, t] ; \dot{B}_{2,1}^{3 / 2}\right)} .
\end{aligned}
$$

Notice that $\left\|u^{n} \cdot \nabla u^{n}\right\|_{L^{1}\left([\tau, t] ; \dot{B}_{2,1}^{3 / 2}\right)} \lesssim \int_{\tau}^{t}\left\|u^{n}\right\|_{\dot{B}_{2,1}^{5 / 2}}\left\|u^{n}\right\|_{\dot{B}_{2,1}^{3 / 2}} \mathrm{~d} t^{\prime}$ and applying the product laws in Besov spaces leads to

$$
\begin{aligned}
& \left\|\left(a^{n}-S_{m} a^{n}\right)\left(\Delta u^{n}-\nabla \Pi^{n}\right)\right\|_{L^{1}\left([\tau, t] ; \dot{B}_{2,1}^{3 / 2}\right)} \\
& \quad \lesssim\left\|a^{n}-\dot{S}_{m} a^{n}\right\|_{L^{\infty}\left([\tau, t] ; \dot{B}_{2,1}^{3 / 2}\right)}\left\|\Delta u^{n}-\nabla \Pi^{n}\right\|_{L^{1}\left([\tau, t] ; \dot{B}_{2,1}^{3 / 2}\right)}, \\
& \left\|\Pi^{n} \nabla \dot{S}_{m} a^{n}\right\|_{L^{1}\left([\tau, t], \dot{B}_{2,1}^{3 / 2}\right)} \lesssim\left\|\nabla \Pi^{n}\right\|_{L^{1}\left([\tau, t] ; \dot{B}_{2,1}^{1 / 2}\right)}\left\|\nabla \dot{S}_{m} a^{n}\right\|_{L^{\infty}\left([\tau, t] ; \dot{B}_{2,1}^{3 / 2}\right)}, \\
& \sum_{q \in \mathbb{Z}} 2^{5 q / 2}\left\|\left[\dot{\Delta}_{q}, \dot{S}_{m} a^{n}\right] \nabla u^{n}\right\|_{L^{1}\left([\tau, t] ; L^{2}\right)} \lesssim\left\|\nabla \dot{S}_{m} a^{n}\right\|_{L^{\infty}\left([\tau, t] ; \dot{B}_{2,1}^{3 / 2}\right)}\left\|u^{n}\right\|_{L^{1}\left([\tau, t] ; \dot{B}_{2,1}^{5 / 2}\right)} \\
& \sum_{q \in \mathbb{Z}} 2^{3 q / 2}\left\|\left[\dot{\Delta}_{q}, \dot{S}_{m} a^{n}\right] \nabla \Pi^{n}\right\|_{L^{1}\left([\tau, t] ; L^{2}\right)} \lesssim\left\|\nabla \dot{S}_{m} a^{n}\right\|_{L^{\infty}\left([\tau, t] ; \dot{B}_{2,1}^{3 / 2}\right)}\left\|\nabla \Pi^{n}\right\|_{L^{1}\left([\tau, t] ; \dot{B}_{2,1}^{1 / 2}\right)}
\end{aligned}
$$

and

$$
\left\|\nabla \dot{S}_{m} a^{n} \cdot \nabla u^{n}\right\|_{L^{1}\left([\tau, t] ; \dot{B}_{2,1}^{3 / 2}\right)} \lesssim\left\|\nabla \dot{S}_{m} a^{n}\right\|_{L^{\infty}\left([\tau, t] ; \dot{B}_{2,1}^{3 / 2}\right)}\left\|\nabla u^{n}\right\|_{L^{1}\left([\tau, t] ; \dot{B}_{2,1}^{3 / 2}\right)} .
$$

Applying Lemma 1 gives rise to

$$
\begin{aligned}
& \left\|\dot{T}_{\Delta u^{n}} \dot{S}_{m} a^{n}\right\|_{L^{1}\left([\tau, t] ; \dot{B}_{2,1}^{3 / 2}\right)}+\left\|\dot{T}_{\nabla \dot{S}_{m} a^{n}} \Delta u^{n}\right\|_{L^{1}\left([\tau, t] ; \dot{B}_{2,1}^{1 / 2}\right)} \\
& \quad+\left\|\dot{\mathcal{R}}\left(\dot{S}_{m} a^{n}, \Delta u^{n}\right)\right\|_{L^{1}\left([\tau, t] ; \dot{B}_{2,1}^{3 / 2}\right)} \lesssim\left\|\nabla \dot{S}_{m} a^{n}\right\|_{L^{\infty}\left([\tau, t] ; \dot{B}_{2,1}^{3 / 2}\right)}\left\|\Delta u^{n}\right\|_{L^{1}\left([\tau, t] ; \dot{B}_{2,1}^{1 / 2}\right)} .
\end{aligned}
$$

Hence, thanks to (4.47) and the fact that

$$
\left\|\nabla \dot{S}_{m} a^{n}\right\|_{L^{\infty}\left([\tau, t] ; \dot{B}_{2,1}^{3 / 2}\right)} \leqq C 2^{5 m / 2}\left\|a_{0}\right\|_{L^{2}},
$$

we infer

$$
\begin{aligned}
& \left\|u^{n}\right\|_{\widetilde{L}^{\infty}\left([\tau, t] ; \dot{B}_{2,1}^{3 / 2}\right)}+\left\|u^{n}\right\|_{L^{1}\left([\tau, t] ; \dot{B}_{2,1}^{7 / 2}\right)}+\left\|\nabla \Pi^{n}\right\|_{L^{1}\left([\tau, t] ; \dot{B}_{2,1}^{3 / 2}\right)} \\
& \lesssim\left\|u^{n}(\tau)\right\|_{\dot{B}_{2,1}^{3 / 2}}+2^{5 m / 2}\left\|a_{0}\right\|_{L^{2}}\left(\left\|\nabla \Pi^{n}\right\|_{L^{1}\left([\tau, t] ; \dot{B}_{2,1}^{1 / 2}\right)}+\left\|u^{n}\right\|_{L^{1}\left([\tau, t] ; \dot{B}_{2,1}^{5 / 2}\right)}\right) \\
& \quad+\left\|a^{n}-\dot{S}_{m} a^{n}\right\|_{L^{\infty}\left([\tau, t] ; \dot{B}_{2,1}^{3 / 2}\right)}\left\|\Delta u^{n}-\nabla \Pi^{n}\right\|_{L^{1}\left([\tau, t] ; \dot{B}_{2,1}^{3 / 2}\right)} \\
& \quad+\int_{\tau}^{t}\left\|u^{n}\right\|_{\dot{B}_{2,1}^{5 / 2}}\left\|u^{n}\right\|_{\dot{B}_{2,1}^{3 / 2}} \mathrm{~d} t^{\prime}
\end{aligned}
$$

from which, with (4.20) and (4.32), we get by applying Gronwall's inequality that

$$
\begin{aligned}
& \left\|u^{n}\right\|_{\widetilde{L}^{\infty}\left([\tau, t] ; \dot{B}_{2,1}^{3 / 2}\right)}+\left\|u^{n}\right\|_{L^{1}\left([\tau, t] ; \dot{B}_{2,1}^{7 / 2}\right)}+\left\|\nabla \Pi^{n}\right\|_{L^{1}\left([\tau, t] ; \dot{B}_{2,1}^{3 / 2}\right)} \\
& \quad \leqq C\left(\| a _ { 0 } \| _ { B _ { 2 , 1 } ^ { 3 / 2 } } \left(\left\|u^{n}(\tau)\right\|_{\dot{B}_{2,1}^{3 / 2}}+\left\|u^{n}(\tau)\right\|_{\left.\dot{B}_{2,1}^{1 / 2}\right)} \exp \left\{C\left\|u^{n}\right\|_{L^{1}\left([\tau, t] ; \dot{B}_{2,1}^{5 / 2}\right)}\right\} .\right.\right.
\end{aligned}
$$


Integrating the above inequality for $\tau$ over $\left[0, t_{0}\right]$, and then dividing the resulting inequality by $t_{0}$ and using (4.1) leads to

$$
\begin{aligned}
& \left\|u^{n}\right\|_{\widetilde{L}^{\infty}\left(\left[t_{0}, t\right] ; \dot{B}_{2,1}^{3 / 2}\right)}+\left\|u^{n}\right\|_{L^{1}\left(\left[t_{0}, t\right] ; \dot{B}_{2,1}^{7 / 2}\right)}+\left\|\nabla \Pi^{n}\right\|_{L^{1}\left(\left[t_{0}, t\right] ; \dot{B}_{2,1}^{3 / 2}\right)} \\
& \quad \leqq C\left(\| a _ { 0 } \| _ { B _ { 2 , 1 } ^ { 3 / 2 } ) } \left(\left\|u^{n}\right\|_{L^{1}\left(\left[0, t_{0}\right] ; \dot{B}_{2,1}^{3 / 2}\right)} / t_{0}+\left\|u_{0}\right\|_{\left.\dot{B}_{2,1}^{1 / 2}\right) \exp }\left\{C\left\|u_{0}\right\|_{\dot{B}_{2,1}^{1 / 2}}\right\}\right.\right. \\
& \quad \leqq C\left(\left\|a_{0}\right\|_{\left.B_{2,1}^{3 / 2}\right)}\left\|u_{0}\right\|_{\dot{B}_{2,1}^{1 / 2}}\left(1+1 / \sqrt{t_{0}}\right) \exp \left\{C\left\|u_{0}\right\|_{\dot{B}_{2,1}^{1 / 2}}\right\} .\right.
\end{aligned}
$$

This along with the compactness argument in Step 3 of Section 4.1 implies (4.2), and we thus complete the proof of Theorem 2 .

\section{Global Wellposedness of (1.2)}

The goal of this section is to prove the global wellposedness part of Theorem 1 , provided that $\left\|u_{0}\right\|_{\dot{B}_{2,1}^{1 / 2}}$ is sufficiently small. More precisely

Theorem 3. Under the assumptions of Theorem 1, (1.2) has a unique global solution $(a, u, \Pi)$ satisfying (1.5) provided that $\left\|u_{0}\right\|_{\dot{B}_{2,1}^{1 / 2}}$ is sufficiently small.

Remark 9. We shall provide more information for this global solution $(a, u, \Pi)$ in the process of proving Theorem 3. Indeed, let $t_{1}$ be determined by (1.8); we shall prove (1.3) has a unique global solution $(\rho, u, \Pi)$ on $\left[t_{1}, \infty\right)$ with $u=v+w$ and $v$ solving (1.9), $w$ solving (1.10). The detailed information of $v$ is presented in Proposition 7, and that of $w$ is in Lemmas 4-6.

Proof of Theorem 3. Thanks to Theorem 2, we conclude that, given $a_{0} \in$ $B_{2,1}^{3 / 2}\left(\mathbb{R}^{3}\right), u_{0} \in \dot{B}_{2,1}^{1 / 2}\left(\mathbb{R}^{3}\right)$ with $\left\|u_{0}\right\|_{\dot{B}_{2,1}^{1 / 2}}$ sufficiently small, (1.2) has a unique local solution $(a, u)$ satisfying $a \in \mathcal{C}\left(\left[0, T^{*}\right) ; B_{2,1}^{3 / 2}\left(\mathbb{R}^{3}\right)\right)$ and $u \in \mathcal{C}\left(\left[0, T^{*}\right) ; \dot{B}_{2,1}^{1 / 2}\left(\mathbb{R}^{3}\right)\right)$ $\cap L_{\text {loc }}^{1}\left(\left(0, T^{*}\right) ; \dot{B}_{2,1}^{5 / 2}\left(\mathbb{R}^{3}\right)\right)$ for some $T^{*}>1$. Our aim in what follows is to prove that $T^{*}=\infty$.

Notice from (1.8) that $\left\|u\left(t_{1}\right)\right\|_{\dot{B}_{2,1}^{1 / 2} \cap \dot{B}_{2,1}^{7 / 2}}$ is very small, provided that $\left\|u_{0}\right\|_{\dot{B}_{2,1}^{1 / 2}}$ is sufficiently small. Let $v$ solve the classical Navier-Stokes system (1.9). As $u\left(t_{1}\right)$ is sufficient small in $\dot{B}_{2,1}^{1 / 2}\left(\mathbb{R}^{3}\right)$, it follows from the classical theory of Navier-Stokes equations [6] that (1.9) has a unique global solution $v \in \mathcal{C}\left(\left[t_{1},+\infty\right) ; \dot{B}_{2,1}^{1 / 2}\right) \cap$ $L^{1}\left(\left[t_{1},+\infty\right) ; \dot{B}_{2,1}^{5 / 2}\right)$ satisfying

$$
\begin{aligned}
& \|v\|_{\widetilde{L}^{\infty}\left(\left[t_{1},+\infty\right) ; \dot{B}_{2,1}^{1 / 2}\right)}+\left\|\left(\Delta v, \nabla \Pi_{v}\right)\right\|_{L^{1}\left(\left[t_{1},+\infty\right) ; \dot{B}_{2,1}^{1 / 2}\right)} \lesssim\left\|u\left(t_{1}\right)\right\|_{\dot{B}_{2,1}^{1 / 2}}, \\
& \left\|\partial_{t} v\right\|_{L^{1}\left(\left[t_{1},+\infty\right) ; \dot{B}_{2,1}^{1 / 2}\right)} \lesssim\left\|u\left(t_{1}\right)\right\|_{\dot{B}_{2,1}^{1 / 2}}+\|\operatorname{div}(v \otimes v)\|_{L^{1}\left(\left[t_{1},+\infty\right) ; \dot{B}_{2,1}^{1 / 2}\right)} \\
& \quad \lesssim\left\|u\left(t_{1}\right)\right\|_{\dot{B}_{2,1}^{1 / 2}}
\end{aligned}
$$


With $v$ thus obtained, we denote $w \stackrel{\text { def }}{=} u-v$. Then thanks to (1.3) and (1.9), $w$ solves (1.10). The proof of Theorem 3 then reduces to proving the global wellposedness of (1.10). For simplicity, in what follows, we just present the a priori estimates for smooth enough solutions of (1.10) on $\left[0, T^{*}\right)$.

\subsection{The Higher Regularities of $v$}

Proposition 7. Let $\left(v, \Pi_{v}\right)$ be the unique global solution of (1.9) which satisfies (5.1). Then for $s_{1} \in\left[\frac{3}{2}, \frac{7}{2}\right]$ and $s_{2} \in\left[\frac{1}{2}, \frac{3}{2}\right]$, there holds

$$
\|v\|_{\widetilde{L}^{\infty}\left(\left[t_{1},+\infty\right) ; \dot{B}_{2,1}^{\left.s_{1}\right)}\right.}+\left\|\left(\Delta v, \nabla \Pi_{v}\right)\right\|_{L^{1}\left(\left[t_{1},+\infty\right) ; \dot{B}_{2,1}^{s_{1}}\right)} \leqq C\left\|u_{0}\right\|_{\dot{B}_{2,1}^{1 / 2}}
$$

and

$$
\left\|\partial_{t} v\right\|_{\widetilde{L}^{\infty}\left(\left[t_{1},+\infty\right) ; \dot{B}_{2,1}^{\left.s_{2}\right)}\right.}+\left\|\left(\partial_{t} \Delta v, \partial_{t} \nabla \Pi_{v}\right)\right\|_{L^{1}\left(\left[t_{1},+\infty\right) ; \dot{B}_{2,1}^{s_{2}}\right)} \leqq C\left\|u_{0}\right\|_{\dot{B}_{2,1}^{1 / 2}}
$$

The proof of this proposition is rather standard. For completeness, we shall outline its proof in the Appendix A.

An immediate corollary of Lemma 1, Proposition 7, and (1.8) leads to

Corollary 1. Under the assumptions of Proposition 7, one has

$$
\|\nabla v\|_{L^{2}\left(\left[t_{1},+\infty\right) ; L^{\infty}\right)}+\left\|\Delta v-\nabla \Pi_{v}\right\|_{L^{2}\left(\left[t_{1},+\infty\right) ; L^{\infty}\right)} \leqq C\left\|u_{0}\right\|_{\dot{B}_{2,1}^{1 / 2}} .
$$

\section{2. $L^{2}$ Estimate of $w$}

Lemma 4. Under the assumptions of Theorem 3, there holds for $t_{1}<t<T^{*}$

$$
\|w\|_{L^{\infty}\left(\left[t_{1}, t\right] ; L^{2}\right)}+\|\nabla w\|_{L^{2}\left(\left[t_{1}, t\right] ; L^{2}\right)} \leqq C\left\|u_{0}\right\|_{\dot{B}_{2,1}^{1 / 2}}
$$

with $C$ being independent of $t$.

Proof. Firstly, thanks to (1.4), one deduces from the transport equation of (1.10) that

$$
\left(1+\left\|a_{0}\right\|_{\dot{B}_{2,1}^{3 / 2}}\right)^{-1} \leqq \rho(t, x) \leqq \underline{b}^{-1},
$$

from which and $1-\rho=\rho a$, we get by applying a standard energy estimate to the $w$ equation of (1.10) that

$$
\begin{aligned}
& \frac{1}{2} \frac{\mathrm{d}}{\mathrm{d} t}\|\sqrt{\rho} w\|_{L^{2}}^{2}+\|\nabla w\|_{L^{2}}^{2}=\int_{\mathbb{R}^{3}}\left((1-\rho)\left(\partial_{t} v+v \cdot \nabla v\right)+\rho w \cdot \nabla v\right) \cdot w \mathrm{~d} x \\
& \quad \leqq C\left(\|\sqrt{\rho} w\|_{L^{2}}\|a\|_{L^{2}}\left\|\partial_{t} v+v \cdot \nabla v\right\|_{L^{\infty}}+\|\sqrt{\rho} w\|_{L^{2}}^{2}\|\nabla v\|_{L^{\infty}}\right),
\end{aligned}
$$

from which, we infer for $t \in\left(t_{1}, T^{*}\right)$ that

$$
\begin{aligned}
& \frac{\mathrm{d}}{\mathrm{d} t}\left(\mathrm{e}^{-2 \int_{t_{1}}^{t}\|\nabla v(\tau)\|_{L^{\infty}} \mathrm{d} \tau}\|\sqrt{\rho} w(t)\|_{L^{2}}^{2}\right) \\
& \quad \leqq C\left\|a_{0}\right\|_{L^{2}} \mathrm{e}^{-2 \int_{t_{1}}^{t}\|\nabla v(\tau)\|_{L^{\infty}} \mathrm{d} \tau}\|\sqrt{\rho} w\|_{L^{2}}\left\|\Delta v-\nabla \Pi_{v}\right\|_{\dot{B}_{2,1}^{3 / 2}}
\end{aligned}
$$


This, along with (1.8) and (5.2), implies

$$
\begin{aligned}
\|\sqrt{\rho} w\|_{L^{\infty}\left(\left[t_{1}, t\right] ; L^{2}\right)}^{2} & \leqq C \mathrm{e}^{\int_{t_{1}}^{t}\|\nabla v(\tau)\|_{L^{\infty}} \mathrm{d} \tau}\left\|\Delta v-\nabla \Pi_{v}\right\|_{L^{1}\left(\left[t_{1}, t\right], \dot{B}_{2,1}^{3 / 2}\right)} \\
& \leqq C\left\|u\left(t_{1}\right)\right\|_{\dot{B}_{2,1}^{3 / 2}} \exp \left\{C\left\|u\left(t_{1}\right)\right\|_{\dot{B}_{2,1}^{1 / 2}}\right\} \leqq C\left\|u_{0}\right\|_{\dot{B}_{2,1}^{1 / 2}} .
\end{aligned}
$$

Plugging the above estimate into (5.7) gives rise to

$$
\|\nabla w\|_{L^{2}\left(\left[t_{1}, t\right] ; L^{2}\right)} \leqq C\left\|u_{0}\right\|_{\dot{B}_{2,1}^{1 / 2}} .
$$

This completes the proof of Lemma 4.

Remark 10. Here we should point out that instead of setting $\mathfrak{w} \stackrel{\text { def }}{=} u-v_{L}$ with $v_{L}(t)=\mathrm{e}^{\left(t-t_{1}\right) \Delta_{1}} u\left(t_{1}\right)$, as in many cases of classical Navier-Stokes system ([7] for instance), we choose to set $w \stackrel{\text { def }}{=} u-v$ with $v$ being determined by (1.9). Indeed if we use $\mathfrak{w} \stackrel{\text { def }}{=} u-v_{L}$, then $\mathfrak{w}$ solves

$$
\left\{\begin{array}{l}
\partial_{t} \rho+\operatorname{div}\left(\rho\left(v_{L}+\mathfrak{w}\right)\right)=0, \quad \operatorname{div} \mathfrak{w}=0, \\
\rho \partial_{t} \mathfrak{w}+\rho\left(v_{L}+\mathfrak{w}\right) \cdot \nabla \mathfrak{w}-\Delta \mathfrak{w}+\nabla \Pi_{\mathfrak{w}} \\
\quad=(1-\rho) \partial_{t} v_{L}-\rho v_{L} \cdot \nabla v_{L}-\rho \mathfrak{w} \cdot \nabla v_{L}, \\
\left.\rho\right|_{t=t_{1}}=\rho\left(t_{1}\right),\left.\quad \mathfrak{w}\right|_{t=t_{1}}=0 .
\end{array}\right.
$$

To perform the $L^{2}$ energy estimate for $\mathfrak{w}$, we need $\rho v_{L} \cdot \nabla v_{L} \in L^{1}\left(\mathbb{R}^{+} ; L^{2}\left(\mathbb{R}^{3}\right)\right)$, which requires $u_{0} \in \dot{B}_{2,1}^{1 / 4}\left(\mathbb{R}^{3}\right)$. Yet $u_{0}$ only belongs to $\dot{B}_{2,1}^{1 / 2}\left(\mathbb{R}^{3}\right)$. This forbids us to use the classical replacement that $\mathfrak{w} \stackrel{\text { def }}{=} u-v_{L}$.

\section{3. $H^{1}$ Estimate of $w$}

With the fundamental estimate (5.5), motivated by [2], we can perform the $H^{1}$ estimate for $w$.

Lemma 5. Under the assumptions of Theorem 3, there exist two positive constants $e_{1}, e_{2}$ so that for $t \in\left[t_{1}, T^{*}\right)$

$$
\begin{aligned}
& \|\nabla w\|_{L^{\infty}\left(\left[t_{1}, t\right], L^{2}\right)}^{2}+\int_{t_{1}}^{t}\left(e_{1}\left\|\partial_{t} w\right\|_{L^{2}}^{2}+e_{2}\left\|\nabla^{2} w\right\|_{L^{2}}^{2}+\left\|\nabla \Pi_{w}\right\|_{L^{2}}^{2}\right) d t^{\prime} \\
& \quad \leqq C\left\|u_{0}\right\|_{\dot{B}_{2,1}^{1 / 2}}^{2}
\end{aligned}
$$

with $C$ being independent of $t$.

Proof. We first get, by taking the $L^{2}$ inner product of the $w$ equation of (1.10) with $\frac{1}{\rho} \Delta w$, and using (5.6),

$$
\begin{aligned}
& \frac{1}{2} \frac{\mathrm{d}}{\mathrm{d} t}\|\nabla w\|_{L^{2}}^{2}+\left\|\frac{1}{\sqrt{\rho}} \Delta w\right\|_{L^{2}}^{2} \lesssim\left\|\frac{1}{\sqrt{\rho}} \Delta w\right\|_{L^{2}}\left\{\|\nabla w\|_{L^{2}}\|v\|_{L^{\infty}}+\|\nabla w\|_{L^{6}}\|w\|_{L^{3}}\right. \\
& \left.\quad+\|w\|_{L^{2}}\|\nabla v\|_{L^{\infty}}+\left\|\nabla \Pi_{w}\right\|_{L^{2}}+\|a\|_{L^{2}}\left\|\partial_{t} v+v \cdot \nabla v\right\|_{L^{\infty}}\right\}
\end{aligned}
$$


which implies

$$
\begin{aligned}
& \frac{\mathrm{d}}{\mathrm{d} t}\|\nabla w\|_{L^{2}}^{2}+\left\|\frac{1}{\sqrt{\rho}} \Delta w\right\|_{L^{2}}^{2} \lesssim\|\nabla w\|_{L^{2}}^{2}\|v\|_{L^{\infty}}^{2}+\|\Delta w\|_{L^{2}}^{2}\|w\|_{L^{3}}^{2} \\
& \quad+\|w\|_{L^{2}}^{2}\|\nabla v\|_{L^{\infty}}^{2}+\left\|\nabla \Pi_{w}\right\|_{L^{2}}^{2}+\left\|\Delta v-\nabla \Pi_{v}\right\|_{L^{\infty}}^{2} .
\end{aligned}
$$

Again, thanks to the $w$ equation of (1.10) and $\operatorname{div} w=0$, one has

$$
\begin{aligned}
& \|\Delta w\|_{L^{2}}+\left\|\nabla \Pi_{w}\right\|_{L^{2}} \leqq \sqrt{2}\left\|\Delta w-\nabla \Pi_{w}\right\|_{L^{2}} \\
& \leqq C\left\{\left\|\sqrt{\rho} \partial_{t} w(t)\right\|_{L^{2}}+C\|w(t)\|_{L^{3}}\|\Delta w(t)\|_{L^{2}}+\|v\|_{L^{\infty}}\|\nabla w\|_{L^{2}}\right. \\
& \left.\quad+\left\|\Delta v-\nabla \Pi_{v}\right\|_{L^{\infty}}+\|w\|_{L^{2}}\|\nabla v\|_{L^{\infty}}\right\} .
\end{aligned}
$$

As a consequence, we obtain for some positive constant $c_{1}$

$$
\begin{aligned}
& \frac{\mathrm{d}}{\mathrm{d} t}\|\nabla w\|_{L^{2}}^{2}+c_{1}\left\|\nabla^{2} w\right\|_{L^{2}}^{2} \leqq C\left\{\|w\|_{L^{3}}^{2}\left\|\nabla^{2} w\right\|_{L^{2}}^{2}+\left\|\sqrt{\rho} \partial_{t} w\right\|_{L^{2}}^{2}\right. \\
& \left.\quad+\|\nabla w\|_{L^{2}}^{2}\|v\|_{L^{\infty}}^{2}+\|w\|_{L^{2}}^{2}\|\nabla v\|_{L^{\infty}}^{2}+\left\|\Delta v-\nabla \Pi_{v}\right\|_{L^{\infty}}^{2}\right\} .
\end{aligned}
$$

Along the same line, we get by taking the $L^{2}$ inner-product of the $w$ equation of (1.10) with $\partial_{t} w$ that

$$
\begin{aligned}
\frac{\mathrm{d}}{\mathrm{d} t}\|\nabla w\|_{L^{2}}^{2}+\left\|\sqrt{\rho} \partial_{t} w\right\|_{L^{2}}^{2} \leqq & C\left(\|w\|_{L^{3}}^{2}\left\|\nabla^{2} w\right\|_{L^{2}}^{2}+\|\nabla w\|_{L^{2}}^{2}\|v\|_{L^{\infty}}^{2}\right. \\
& \left.+\|w\|_{L^{2}}^{2}\|\nabla v\|_{L^{\infty}}^{2}+\left\|\Delta v-\nabla \Pi_{v}\right\|_{L^{\infty}}^{2}\right)
\end{aligned}
$$

Thanks to (5.10) and (5.11), we conclude that there is a positive constant $c_{2}$ such that

$$
\begin{aligned}
& \frac{\mathrm{d}}{\mathrm{d} t}\|\nabla w\|_{L^{2}}^{2}+c_{2}\left\|\partial_{t} w\right\|_{L^{2}}^{2}+\left(\frac{c_{1}}{2 C_{2}}-C_{2}\|w\|_{L^{3}}^{2}\right)\left\|\nabla^{2} w\right\|_{L^{2}}^{2} \\
& \quad \leqq C_{3}\left(\|\nabla w\|_{L^{2}}^{2}\|v\|_{L^{\infty}}^{2}+\|w\|_{L^{2}}^{2}\|\nabla v\|_{L^{\infty}}^{2}+\left\|\Delta v-\nabla \Pi_{v}\right\|_{L^{\infty}}^{2}\right) .
\end{aligned}
$$

Now let $\tau^{*}$ be determined by

$$
\tau^{*} \stackrel{\text { def }}{=} \sup \left\{t \geqq t_{1},\|w(t)\|_{L^{3}}^{2} \leqq \frac{c_{1}}{2 C_{2}^{2}}\right\} .
$$

We claim that $\tau^{*}=T^{*}$, provided that $\left\|u_{0}\right\|_{\dot{B}_{2,1}^{1 / 2}}$ is sufficiently small. Otherwise for $t \in\left[t_{1}, \tau^{*}\right)$, it follows from (5.12) that

$$
\begin{aligned}
& \frac{\mathrm{d}}{\mathrm{d} t}\|\nabla w\|_{L^{2}}^{2}+c_{2}\left\|\partial_{t} w\right\|_{L^{2}}^{2}+\frac{c_{1}}{4 C_{2}}\left\|\nabla^{2} w\right\|_{L^{2}}^{2} \\
& \quad \leqq C_{3}\left(\|\nabla w\|_{L^{2}}^{2}\|v\|_{L^{\infty}}^{2}+\|w\|_{L^{2}}^{2}\|\nabla v\|_{L^{\infty}}^{2}+\left\|\Delta v-\nabla \Pi_{v}\right\|_{L^{\infty}}^{2}\right) .
\end{aligned}
$$

Applying Gronwall's inequality to (5.14) and using (5.4) gives rise to

$$
\begin{aligned}
\|\nabla w\|_{L^{2}}^{2} & \leqq C_{3} \exp \left\{C_{3} \int_{t_{1}}^{t}\|v\|_{L^{\infty}}^{2} \mathrm{~d} t^{\prime}\right\} \int_{t_{1}}^{t}\left(\|w\|_{L^{2}}^{2}\|\nabla v\|_{L^{\infty}}^{2}+\left\|\Delta v-\nabla \Pi_{v}\right\|_{L^{\infty}}^{2}\right) \mathrm{d} t^{\prime} \\
& \leqq C_{4}\left\|u_{0}\right\|_{\dot{B}_{2,1}^{1 / 2}}^{2}
\end{aligned}
$$


However, notice from (5.5) and (5.15) that

$$
\|w(t)\|_{L^{3}}^{2} \leqq C\|w(t)\|_{L^{2}}\|\nabla w(t)\|_{L^{2}} \leqq C_{5}\left\|u_{0}\right\|_{\dot{B}_{2,1}^{1 / 2}}^{2} \leqq \frac{c_{1}}{4 C_{2}^{2}} \quad \text { for } t \in\left[t_{1}, \tau^{*}\right),
$$

provided that $\left\|u_{0}\right\|_{\dot{B}_{2,1}^{1 / 2}}^{2} \leqq \frac{c_{1}}{4 C_{2}^{2} C_{5}}$, which contradicts (5.13). This, in turn, shows that $\tau^{*}=T^{*}$. Then integrating (5.14) and using (5.4) leads to (5.9). This completes the proof of the lemma.

\section{4. $H^{2}$ Estimate of $w$}

Lemma 6. Under the assumptions of Theorem 3, there exists a time independent constant $C$ such that for $t \in\left[t_{1}, T^{*}\right)$

$$
\left\|\nabla^{2} w\right\|_{L^{\infty}\left(\left[t_{1}, t\right], L^{2}\right)}+\left\|\nabla w_{t}\right\|_{L^{2}\left(\left[t_{1}, t\right], L^{2}\right)}+\left\|\nabla^{2} w\right\|_{L^{2}\left(\left[t_{1}, t\right], L^{6}\right)} \leqq C .
$$

Proof. Step 1. Energy estimate of $\sqrt{\rho} w_{t}$.

We get by first applying $\partial_{t}$ to the $w$ equation of (1.10) and then taking the $L^{2}$ inner product of the resulting equation with $\partial_{t} w$, that

$$
\begin{aligned}
& \frac{1}{2} \frac{\mathrm{d}}{\mathrm{d} t}\left\|\sqrt{\rho} w_{t}\right\|_{L^{2}}^{2}+\left\|\nabla w_{t}\right\|_{L^{2}}^{2}=\int_{\mathbb{R}^{3}}(1-\rho) w_{t} \cdot \partial_{t}\left(\Delta v-\nabla \Pi_{v}\right) \mathrm{d} x \\
& -\int_{\mathbb{R}^{3}} \rho_{t} w_{t} \cdot\left(w_{t}+(w+v) \cdot \nabla w+w \cdot \nabla v+\left(\Delta v-\nabla \Pi_{v}\right)\right) \mathrm{d} x \\
& -\int_{\mathbb{R}^{3}} \rho w_{t} \cdot\left((v+w)_{t} \cdot \nabla w+w_{t} \cdot \nabla v+w \cdot \nabla v_{t}\right) \mathrm{d} x \stackrel{\text { def }}{=} I+I I+I I I,
\end{aligned}
$$

where we used $\rho_{t}=-\operatorname{div}(\rho(w+v))$ so that

$$
\frac{1}{2} \int_{\mathbb{R}^{3}} \rho_{t}\left|w_{t}\right|^{2} \mathrm{~d} x=\int_{\mathbb{R}^{3}} \rho w_{t} \cdot[(w+v) \cdot \nabla] w_{t} \mathrm{~d} x .
$$

First, one has

$$
\begin{aligned}
|I| & \lesssim\left\|\sqrt{\rho} w_{t}\right\|_{L^{2}}\|a\|_{L^{\infty}}\left\|\partial_{t}\left(\Delta v-\nabla \Pi_{v}\right)\right\|_{L^{2}} \\
& \lesssim\left\|a_{0}\right\|_{L^{\infty}}\left\|\sqrt{\rho} w_{t}\right\|_{L^{2}}\left\|\partial_{t}\left(\Delta v-\nabla \Pi_{v}\right)\right\|_{L^{2}} .
\end{aligned}
$$

While again thanks to the transport equation of (1.10), one gets by using integration by parts

$$
\begin{aligned}
-I I= & \int_{\mathbb{R}^{3}} \rho(v+w)^{i} w_{t} \cdot\left(\partial_{i}(w+v) \cdot \nabla w+(w+v) \cdot \nabla \partial_{i} w+\partial_{i} w \cdot \nabla v\right. \\
& \left.+w \cdot \nabla \partial_{i} v\right) \mathrm{d} x+\int_{\mathbb{R}^{3}} \rho(v+w)^{i} w_{t} \cdot \partial_{i}\left(\Delta v-\nabla \Pi_{v}\right) \mathrm{d} x \\
& +\int_{\mathbb{R}^{3}} \rho(v+w)^{i} \partial_{i} w_{t} \cdot\left((w+v) \cdot \nabla w+w \cdot \nabla v+\left(\Delta v-\nabla \Pi_{v}\right)\right) \mathrm{d} x \\
& +2 \int_{\mathbb{R}^{3}} \rho w_{t} \cdot\left((w+v) \cdot \nabla w_{t}\right) \mathrm{d} x \stackrel{\text { def }}{=} I I_{1}+I I_{2}+I I_{3} .
\end{aligned}
$$


Yet thanks to (5.1), (5.2) and (5.9), one has

$$
\|v+w\|_{L^{6}} \lesssim\|\nabla v\|_{L^{2}}+\|\nabla w\|_{L^{2}} \leqq C,
$$

from which, we deduce that

$$
\begin{aligned}
\left|I I_{1}\right| \leqq & \left\|w_{t}\right\|_{L^{6}}\|v+w\|_{L^{6}}\left(\|\nabla(v+w)\|_{L^{6}}\|\nabla w\|_{L^{2}}+\|v+w\|_{L^{6}}\left\|\nabla^{2} w\right\|_{L^{2}}\right. \\
& \left.+\|\nabla v\|_{L^{6}}\|\nabla w\|_{L^{2}}+\left\|\nabla^{2} v\right\|_{L^{6}}\|w\|_{L^{2}}\right) \\
\leqq & \frac{1}{8}\left\|\nabla w_{t}\right\|_{L^{2}}^{2}+C\left(\|\Delta w\|_{L^{2}}^{2}+\|\nabla v\|_{L^{6}}^{2}+\left\|\nabla^{2} v\right\|_{L^{6}}^{2}\right) .
\end{aligned}
$$

While it follows from (5.1), (5.5), (5.9) and

$$
\|v+w\|_{L^{4}} \lesssim\|v\|_{\dot{B}_{2,1}^{3 / 4}}+\|w\|_{L^{2}}^{1 / 4}\|\nabla w\|_{L^{2}}^{3 / 4} \leqq C,
$$

that

$$
\begin{aligned}
\left|I I_{2}\right| \leqq & \left\|\sqrt{\rho} w_{t}\right\|_{L^{2}}\left(\|v+w\|_{L^{4}}\left\|\nabla\left(\Delta v-\nabla \Pi_{v}\right)\right\|_{L^{4}}+\|v+w\|_{L^{\infty}}\left\|\nabla w_{t}\right\|_{L^{2}}\right) \\
\leqq & \frac{1}{16}\left\|\nabla w_{t}\right\|_{L^{2}}^{2}+C\left(\left\|\sqrt{\rho} w_{t}\right\|_{L^{2}}^{2}\left(\|v\|_{L^{\infty}}^{2}+\|\nabla w\|_{L^{2}}\left\|\nabla^{2} w\right\|_{L^{2}}\right)\right. \\
& \left.+\left\|\sqrt{\rho} w_{t}\right\|_{L^{2}}\left\|\nabla\left(\Delta v-\nabla \Pi_{v}\right)\right\|_{L^{4}}\right) .
\end{aligned}
$$

Along the same line, we have

$$
\begin{aligned}
\left|I I_{3}\right| \leqq & \left\|\nabla w_{t}\right\|_{L^{2}}\left(\|v+w\|_{L^{\infty}}^{2}\|\nabla w\|_{L^{2}}+\|v+w\|_{L^{6}}\|\nabla v\|_{L^{6}}\|w\|_{L^{6}}\right. \\
& \left.+\|v+w\|_{L^{4}}\left\|\Delta v-\nabla \Pi_{v}\right\|_{L^{4}}\right) \\
\leqq & \frac{1}{16}\left\|\nabla w_{t}\right\|_{L^{2}}^{2}+C\left(\|v\|_{L^{\infty}}^{4}+\|\nabla v\|_{L^{6}}^{2}+\|\Delta w\|_{L^{2}}^{2}+\left\|\Delta v-\nabla \Pi_{v}\right\|_{L^{4}}^{2}\right) .
\end{aligned}
$$

As a consequence, we obtain

$$
\begin{aligned}
|I I| \leqq & C\left\{\|v\|_{L^{\infty}}^{4}+\|\nabla v\|_{L^{6}}^{2}+\|\Delta w\|_{L^{2}}^{2}+\left\|\Delta v-\nabla \Pi_{v}\right\|_{L^{4}}^{2}+\left\|\nabla^{2} v\right\|_{L^{6}}^{2}\right. \\
& +\left\|\sqrt{\rho} w_{t}\right\|_{L^{2}}^{2}\left(\|v\|_{L^{\infty}}^{2}+\|\nabla w\|_{L^{2}}\left\|\nabla^{2} w\right\|_{L^{2}}\right) \\
& \left.+\left\|\sqrt{\rho} w_{t}\right\|_{L^{2}}\left\|\nabla\left(\Delta v-\nabla \Pi_{v}\right)\right\|_{L^{4}}\right\}+\frac{1}{4}\left\|\nabla w_{t}\right\|_{L^{2}}^{2}
\end{aligned}
$$

Similarly, we have

$$
\begin{aligned}
|I I I| \leqq & \left\|\sqrt{\rho} w_{t}\right\|_{L^{2}}\left(\left\|v_{t}\right\|_{L^{\infty}}\|\nabla w\|_{L^{2}}+\left\|\nabla w_{t}\right\|_{L^{6}}\|\nabla w\|_{L^{3}}\right. \\
& \left.+\left\|\sqrt{\rho} w_{t}\right\|_{L^{2}}\|\nabla v\|_{L^{\infty}}+\|w\|_{L^{4}}\left\|\nabla v_{t}\right\|_{L^{4}}\right) \\
\leqq & \frac{1}{16}\left\|\nabla w_{t}\right\|_{L^{2}}^{2}+C\left\{\left\|\sqrt{\rho} w_{t}\right\|_{L^{2}}^{2}\left(\|\nabla w\|_{L^{2}}\left\|\nabla^{2} w\right\|_{L^{2}}+\|\nabla v\|_{L^{\infty}}\right)\right. \\
& \left.+\left\|\sqrt{\rho} w_{t}\right\|_{L^{2}}\left(\left\|v_{t}\right\|_{L^{\infty}}+\left\|\nabla v_{t}\right\|_{L^{4}}\right)\right\}
\end{aligned}
$$

which together with (5.17), (5.18) and (5.19) yields

$$
\frac{\mathrm{d}}{\mathrm{d} t}\left\|\sqrt{\rho} w_{t}\right\|_{L^{2}}^{2}+\left\|\nabla w_{t}\right\|_{L^{2}}^{2} \leqq C\left(\left(f_{1}(t)+f_{3}(t)\right)\left\|\sqrt{\rho} w_{t}\right\|_{L^{2}}^{2}+f_{2}(t)+f_{3}(t)\right),
$$


with

$$
\begin{aligned}
& f_{1}(t) \stackrel{\text { def }}{=}\|v\|_{L^{\infty}}^{2}+\|\nabla v\|_{L^{\infty}}+\|\nabla w\|_{L^{2}}\left\|\nabla^{2} w\right\|_{L^{2}}, \\
& f_{2}(t) \stackrel{\text { def }}{=}\|v\|_{L^{\infty}}^{4}+\|\nabla v\|_{L^{6}}^{2}+\|\Delta w\|_{L^{2}}^{2}+\left\|\Delta v-\nabla \Pi_{v}\right\|_{L^{4}}^{2}+\left\|\nabla^{2} v\right\|_{L^{6}}^{2} \text { and } \\
& f_{3}(t) \stackrel{\text { def }}{=}\left\|\nabla\left(\Delta v-\nabla \Pi_{v}\right)\right\|_{L^{4}}+\left\|\partial_{t}\left(\Delta v-\nabla \Pi_{v}\right)\right\|_{L^{p}}+\left\|v_{t}\right\|_{L^{\infty}}+\left\|\nabla v_{t}\right\|_{L^{4}} .
\end{aligned}
$$

Applying Gronwall's inequality to (5.20) yields for $t \in\left(t_{1}, T^{*}\right)$

$$
\begin{aligned}
\| & \sqrt{\rho} w_{t}(t)\left\|_{L^{2}}^{2}+\int_{t_{1}}^{t}\right\| \nabla w_{t}\left(t^{\prime}\right) \|_{L^{2}}^{2} \mathrm{~d} t^{\prime} \\
\leqq & C \exp \left\{C \int_{t_{1}}^{t}\left(f_{1}\left(t^{\prime}\right)+f_{3}\left(t^{\prime}\right)\right) \mathrm{d} t^{\prime}\right\} \\
& \times\left(\left\|\left(\sqrt{\rho} w_{t}\right)\left(t_{1}\right)\right\|_{L^{2}}^{2}+\int_{t_{1}}^{t}\left(f_{2}\left(t^{\prime}\right)+f_{3}\left(t^{\prime}\right)\right) \mathrm{d} t^{\prime}\right) .
\end{aligned}
$$

However, noticing that $\dot{B}_{2,1}^{3 / 2} \hookrightarrow L^{\infty}, \dot{B}_{2,1}^{1} \hookrightarrow L^{6}, \dot{B}_{2,1}^{3 / 4} \hookrightarrow L^{4}$, we deduce from (5.2), (5.3), (5.5) and (5.9) that

$$
\int_{t_{1}}^{t}\left(f_{1}\left(t^{\prime}\right)+f_{2}\left(t^{\prime}\right)+f_{3}\left(t^{\prime}\right)\right) \mathrm{d} t^{\prime} \leqq C,
$$

with $C$ being independent of $t$. Whereas taking the $L^{2}$ inner product of the $w$ equation of (1.10) with $w_{t}$ at $t=t_{1}$ and using (4.2) gives rise to

$$
\begin{aligned}
\left\|\left(\sqrt{\rho} w_{t}\right)\left(t_{1}\right)\right\|_{L^{2}} & \leqq C\left\|a\left(t_{1}\right)\right\|_{L^{2}}\left\|\left(\partial_{t} v+v \cdot \nabla v\right)\left(t_{1}\right)\right\|_{\dot{B}_{2,1}^{3 / 2}} \\
& \leqq C\left(\left\|v\left(t_{1}\right)\right\|_{\dot{B}_{2,1}^{7 / 2}}+\left\|v\left(t_{1}\right)\right\|_{\dot{B}_{2,1}^{3 / 2}}\left\|v\left(t_{1}\right)\right\|_{\left.\dot{B}_{2,1}^{5 / 2}\right)} \leqq C .\right.
\end{aligned}
$$

As a consequence, we deduce from (5.21) that

$$
\sup _{t \in\left[t_{1}, T^{*}\right)}\left\|\sqrt{\rho} w_{t}(t)\right\|_{L^{2}}^{2}+\int_{t_{1}}^{T^{*}}\left\|\nabla w_{t}\left(t^{\prime}\right)\right\|_{L^{2}}^{2} \mathrm{~d} t^{\prime} \leqq C .
$$

Step 2. The second space derivative estimate of $w$.

We first observe from the $w$ equation of (1.10) that

$$
\begin{aligned}
& \left\|\nabla^{2} w\right\|_{L^{2}}+\left\|\nabla \Pi_{w}\right\|_{L^{2}} \leqq \frac{1}{2}\left\|\nabla^{2} w\right\|_{L^{2}}+C\left\{\left\|\sqrt{\rho} w_{t}\right\|_{L^{2}}+\|\nabla w\|_{L^{2}}^{3}\right. \\
& \left.\quad+\|v\|_{L^{\infty}}\|\nabla w\|_{L^{2}}+\|\nabla v\|_{L^{\infty}}\|w\|_{L^{2}}+\|v\|_{\dot{B}_{2,1}^{7 / 2}}+\|v\|_{\dot{B}_{2,1}^{3 / 2}}\|v\|_{\dot{B}_{2,1}^{5 / 2}}\right\}
\end{aligned}
$$

which along with (5.2), (5.3), (5.5), (5.9) and (5.22) ensures that

$$
\sup _{t \in\left[t_{1}, T^{*}\right)}\left(\left\|\nabla^{2} w(t)\right\|_{L^{2}}+\left\|\nabla \Pi_{w}(t)\right\|_{L^{2}}\right) \leqq C .
$$

On the other hand, let $(v, q)$ solve

$$
-\Delta v+\nabla q=f, \quad \operatorname{div} v=0 .
$$


Then one has $\nabla q=-\nabla(-\Delta)^{-1} \operatorname{div} f$, and for any $r \in(1, \infty)$,

$$
\|\nabla q\|_{L^{r}} \leqq C\|f\|_{L^{r}} \quad\|\Delta v\|_{L^{r}} \leqq C\|f\|_{L^{r}},
$$

from which and the $w$ equation of (1.10), we infer

$$
\begin{aligned}
& \left\|\nabla^{2} w\right\|_{L^{6}}+\left\|\nabla \Pi_{w}\right\|_{L^{6}} \leqq C\left(\left\|w_{t}\right\|_{L^{6}}+\|w \cdot \nabla w\|_{L^{6}}+\|v \cdot \nabla w\|_{L^{6}}\right. \\
& \left.\quad+\|w \cdot \nabla v\|_{L^{6}}+\|(1-\rho)\|_{L^{6}}\left\|\partial_{t} v+v \cdot \nabla v\right\|_{L^{\infty}}\right),
\end{aligned}
$$

which along with (5.9) implies

$$
\begin{aligned}
& \left\|\nabla^{2} w\right\|_{L^{6}}+\left\|\nabla \Pi_{w}\right\|_{L^{6}} \\
& \leqq C\left(\left\|\nabla w_{t}\right\|_{L^{2}}+\|\nabla w\|_{L^{2}}\left\|\nabla^{2} w\right\|_{L^{2}}^{\frac{1}{2}}\left\|\nabla^{2} w\right\|_{L^{6}}^{\frac{1}{2}}+\left\|\nabla^{2} w\right\|_{L^{2}}\|v\|_{L^{\infty}}\right. \\
& \left.\quad+\|\nabla w\|_{L^{2}}\|\nabla v\|_{L^{\infty}}+\|v\|_{\dot{B}_{2,1}^{7 / 2}}+\|v\|_{\dot{B}_{2,1}^{3 / 2}}\|v\|_{\dot{B}_{2,1}^{5 / 2}}\right) \\
& \leqq \\
& \qquad \frac{1}{2}\left\|\nabla^{2} w\right\|_{L^{6}}+C\left(\left\|\nabla w_{t}\right\|_{L^{2}}+\left\|\nabla^{2} w\right\|_{L^{2}}+\|v\|_{\dot{B}_{2,1}^{7 / 2}}+\|v\|_{\dot{B}_{2,1}^{5 / 2}}\right) .
\end{aligned}
$$

Therefore thanks to (5.5), (5.22) and (5.23), we obtain

$$
\begin{aligned}
& \left\|\nabla^{2} w\right\|_{L^{2}\left(\left[t_{1}, t\right] ; L^{6}\right)}^{2}+\left\|\nabla \Pi_{w}\right\|_{L^{2}\left(\left[t_{1}, t\right] ; L^{6}\right)}^{2} \leqq C\left\{\left\|\nabla w_{t}\right\|_{L^{2}\left(\left[t_{1}, t\right] ; L^{2}\right)}^{2}\right. \\
& \left.\quad+\left\|\nabla^{2} w\right\|_{L^{2}\left(\left[t_{1}, t\right] ; L^{2}\right)}^{2}+\|v\|_{L^{2}\left(\left[t_{1}, t\right] ; \dot{B}_{2,1}^{7 / 2}\right)}^{2}+\|v\|_{L^{2}\left(\left[t_{1}, t\right] ; \dot{B}_{2,1}^{5 / 2}\right)}^{2}\right\} \leqq C .
\end{aligned}
$$

This ends the proof of the lemma.

\subsection{Proof of Theorem 3}

We first rewrite the momentum equation in (1.3) as

$$
\partial_{t} u+u \cdot \nabla u-\Delta u+\nabla \Pi=(1-\rho)\left(\partial_{t} u+u \cdot \nabla u\right) .
$$

Then it follows from the classical theory of Navier-Stokes equations (see [6] for instance) that $t \in\left[t_{1}, T^{*}\right)$

$$
\begin{aligned}
& \|u\|_{\widetilde{L}^{\infty}\left(\left[t_{1}, t\right] ; \dot{B}_{2,1}^{1 / 2}\right)}+\|u\|_{L^{1}\left(\left[t_{1}, t\right] ; \dot{B}_{2,1}^{5 / 2}\right)}+\|\nabla \Pi\|_{L^{1}\left(\left[t_{1}, t\right] ; \dot{B}_{2,1}^{1 / 2}\right)} \\
& \lesssim\left\|u\left(t_{1}\right)\right\|_{\dot{B}_{2,1}^{1 / 2}}+\int_{t_{1}}^{t}\|\nabla u\|_{L^{\infty}\|u\|_{\dot{B}_{2,1}^{1 / 2}} \mathrm{~d} t^{\prime}} \\
& \quad+\left\|(1-\rho)\left(\partial_{t} u+u \cdot \nabla u\right)\right\|_{L^{1}\left(\left[t_{1}, t\right] ; \dot{B}_{2,1}^{1 / 2}\right)}
\end{aligned}
$$

Applying the product law in Besov spaces gives

$$
\begin{aligned}
& \left\|(1-\rho)\left(\partial_{t} u+u \cdot \nabla u\right)\right\|_{L^{1}\left(\left[t_{1}, t\right] ; \dot{B}_{2,1}^{1 / 2}\right)} \\
& \quad \lesssim\|1-\rho\|_{L^{\infty}\left(\left[t_{1}, t\right] ; \dot{B}_{2,1}^{3 / 2}\right)}\left\|\partial_{t} u+u \cdot \nabla u\right\|_{L^{1}\left(\left[t_{1}, t\right] ; \dot{B}_{2,1}^{1 / 2}\right)}
\end{aligned}
$$

Yet thanks to Lemma 1 and (5.16), one has

$$
\left\|\partial_{t} u\right\|_{L^{1}\left(\left[t_{1}, t\right] ; \dot{B}_{2,1}^{1 / 2}\right)} \leqq C\left(t^{1 / 2}\left\|\partial_{t} w\right\|_{L^{2}\left(\left[t_{1}, t\right] ; H^{1}\right)}+\left\|u\left(t_{1}\right)\right\|_{\dot{B}_{2,1}^{1 / 2}}\right) \leqq C\left(t^{1 / 2}+1\right),
$$


and

$$
\|u \cdot \nabla u\|_{L^{1}\left(\left[t_{1}, t\right] ; \dot{B}_{2,1}^{1 / 2}\right)} \leqq C \int_{t_{1}}^{t}\left(\|\nabla w\|_{L^{2}}\|\Delta w\|_{L^{2}}+\|v\|_{\dot{B}_{2,1}^{3 / 2}}^{2}\right) \mathrm{d} t^{\prime} \leqq C .
$$

Thanks to Theorem 2.87 in [4] and Proposition 1, we have

$$
\begin{aligned}
\|1-\rho\|_{\widetilde{L}^{\infty}\left(\left[t_{1}, t\right] ; \dot{B}_{2,1}^{3 / 2}\right)} \lesssim\|a\|_{\widetilde{L}^{\infty}\left(\left[t_{1}, t\right] ; \dot{B}_{2,1}^{3 / 2}\right)} & \\
& \lesssim\left\|a\left(t_{1}\right)\right\|_{\dot{B}_{2,1}^{3 / 2}} \exp \left\{C \int_{t_{1}}^{t}\left\|u\left(t^{\prime}\right)\right\|_{\dot{B}_{6,1}^{3 / 2}} \mathrm{~d} t^{\prime}\right\} .
\end{aligned}
$$

Applying Lemma 1 leads to

$$
\|u\|_{\dot{B}_{6,1}^{3 / 2}} \lesssim\|\nabla w\|_{L^{6}}^{1 / 2}\left\|\nabla^{2} w\right\|_{L^{6}}^{1 / 2}+\|v\|_{\dot{B}_{2,1}^{5 / 2}}
$$

which along with (5.9) and (5.16) implies

$$
\begin{aligned}
& \|\nabla u\|_{L^{1}\left(\left[t_{1}, t\right] ; L^{\infty}\right)}+\|u\|_{L^{1}\left(\left[t_{1}, t\right] ; \dot{B}_{6,1}^{3 / 2}\right)} \leqq C\|u\|_{L^{1}\left(\left[t_{1}, t\right] ; \dot{B}_{6,1}^{3 / 2}\right)} \\
& \quad \lesssim\|v\|_{L^{1}\left(\left[t_{1}, t\right] ; \dot{B}_{2,1}^{5 / 2}\right)}+t^{1 / 2}\|\Delta w\|_{L^{2}\left(\left[t_{1}, t\right] ; L^{2}\right)}^{1 / 2}\|\Delta w\|_{L^{2}\left(\left[t_{1}, t\right] ; L^{6}\right)}^{1 / 2} \lesssim\left(1+t^{1 / 2}\right) .
\end{aligned}
$$

Therefore, we obtain

$$
\left\|(1-\rho)\left(\partial_{t} u+u \cdot \nabla u\right)\right\|_{L^{1}\left(\left[t_{1}, t\right] ; \dot{B}_{2,1}^{1 / 2}\right)} \leqq C\left\|a\left(t_{1}\right)\right\|_{\dot{B}_{2,1}^{3 / 2}} \exp \left\{C t^{1 / 2}\right\} .
$$

Then applying Gronwall's inequality to (5.24) gives rise to

$$
\begin{aligned}
& \|u\|_{\widetilde{L}^{\infty}\left(\left[t_{1}, t\right] ; \dot{B}_{2,1}^{1 / 2}\right)}+\|u\|_{L^{1}\left(\left[t_{1}, t\right] ; \dot{B}_{2,1}^{5 / 2}\right)}+\|\nabla \Pi\|_{L^{1}\left(\left[t_{1}, t\right] ; \dot{B}_{2,1}^{1 / 2}\right)} \\
& \quad \leqq C\left(\left\|u_{0}\right\|_{\dot{B}_{2,1}^{1 / 2}}+\left\|a_{0}\right\|_{\left.B_{2,1}^{3 / 2}\right) \exp \{C \sqrt{t}\} .}\right.
\end{aligned}
$$

From (5.25) and (5.26), we infer by a standard argument that $T^{*}=\infty$. Moreover, the global solution $(a, u, \Pi)$ thus obtained satisfies (1.5). This completes the proof of Theorem 3.

Combining Theorem 2 with Theorem 3, we complete the proof of Theorem 1.

Acknowledgements. Part of this work was done when we were visiting Morningside Center of Mathematics, CAS, in the summer of 2010. We appreciate the hospitality and the financial support from the center. GUI is partially supported by NSF of China under Grant 11001111 and 11171241, and Grants 10JDG141 and 10JDG157 from Jiangsu University. P. ZHANG is partially supported by NSF of China under Grant 10421101 and 10931007, the one hundred talents' plan from Chinese Academy of Sciences under Grant GJHZ200829 and innovation grant from National Center for Mathematics and Interdisciplinary Sciences. 


\section{Appendix A. Outline of the Proof to Proposition 7}

Proof. (Outline of the proof of Proposition 7) Again for simplicity, we just present the a priori estimates for smooth enough solutions of (1.9). Firstly, it is easy to observe that for $s_{1} \in[3 / 2,7 / 2]$,

$$
\begin{aligned}
& \|v\|_{\widetilde{L}^{\infty}\left(\left[t_{1}, t\right) ; \dot{B}_{2,1}^{\left.s_{1}\right)}\right.}+\|v\|_{L^{1}\left(\left[t_{1}, t\right) ; \dot{B}_{2,1}^{s_{1}+2}\right)}+\left\|\nabla \Pi_{v}\right\|_{L^{1}\left(\left[t_{1}, t\right) ; \dot{B}_{2,1}^{s_{1}}\right)} \\
& \quad \leqq\left\|u\left(t_{1}\right)\right\|_{\dot{B}_{2,1}^{s}}+C \int_{t_{1}}^{t}\|v \cdot \nabla v\|_{\dot{B}_{2,1}^{s_{1}}} \mathrm{~d} t^{\prime} \\
& \quad \leqq\left\|u\left(t_{1}\right)\right\|_{\dot{B}_{2,1}^{s_{1}}}+\frac{1}{2}\|v\|_{L^{1}\left(\left[t_{1}, t\right) ; \dot{B}_{2,1}^{s_{1}+2}\right)}+C \int_{t_{1}}^{t}\left(\|v\|_{\dot{B}_{2,1}^{3 / 2}}^{2}+\|v\|_{\left.\dot{B}_{2,1}^{5 / 2}\right)\|v\|_{\dot{B}_{2,1}^{s_{1}}} \mathrm{~d} t^{\prime}}\right.
\end{aligned}
$$

Applying Gronwall's inequality and using (5.1), (1.8) yields

$$
\begin{aligned}
& \|v\|_{\widetilde{L}^{\infty}\left(\left[t_{1},+\infty\right) ; \dot{B}_{2,1}^{\left.s_{1}\right)}\right.}+\|v\|_{L^{1}\left(\left[t_{1},+\infty\right) ; \dot{B}_{2,1}^{s_{1}+2}\right)}+\left\|\nabla \Pi_{v}\right\|_{L^{1}\left(\left[t_{1},+\infty\right) ; \dot{B}_{2,1}^{s_{1}}\right)} \\
& \quad \leqq\left\|u\left(t_{1}\right)\right\|_{\dot{B}_{2,1}^{s}} \exp \left\{C \int_{t_{1}}^{\infty}\left(\|v\|_{\dot{B}_{2,1}^{3 / 2}}^{2}+\|v\|_{\left.\dot{B}_{2,1}^{5 / 2}\right)} \mathrm{d} t^{\prime}\right\} \leqq C\left\|u_{0}\right\|_{\dot{B}_{2,1}^{1 / 2}}\right.
\end{aligned}
$$

Then thanks to (1.9) and (A.1), we infer

$$
\begin{gathered}
\left\|\partial_{t} v\right\|_{\widetilde{L}^{\infty}\left(\left[t_{1},+\infty\right) ; \dot{B}_{2,1}^{1 / 2}\right)}+\left\|\partial_{t} v\right\|_{L^{1}\left(\left[t_{1},+\infty\right) ; \dot{B}_{2,1}^{5 / 2}\right)} \\
+\left\|\partial_{t} \Pi_{v}\right\|_{L^{1}\left(\left[t_{1},+\infty\right), \dot{B}_{2,1}^{3 / 2}\right)} \leqq C\left\|u_{0}\right\|_{\dot{B}_{2,1}^{1 / 2}}
\end{gathered}
$$

Whereas it follows from the standard parabolic estimate that

$$
\begin{aligned}
& \left\|\partial_{t} v\right\|_{\widetilde{L}^{\infty}\left(\left[t_{1},+\infty\right) ; \dot{B}_{2,1}^{3 / 2}\right)}+\left\|\left(\partial_{t} \Delta v, \partial_{t} \nabla \Pi_{v}\right)\right\|_{L^{1}\left(\left[t_{1},+\infty\right) ; \dot{B}_{2,1}^{3 / 2}\right)} \\
& \quad \lesssim\left\|\partial_{t} v\left(t_{1}\right)\right\|_{\dot{B}_{2,1}^{3 / 2}}+\left\|\partial_{t}(v \cdot \nabla v)\right\|_{L^{1}\left(\left[t_{1},+\infty\right) ; \dot{B}_{2,1}^{3 / 2}\right)} .
\end{aligned}
$$

Yet notice that $\left.\partial_{t} v\left(t_{1}\right)=\Delta v\left(t_{1}\right)-\mathbb{P}(v \cdot \nabla) v\right)\left(t_{1}\right)$ with $\mathbb{P}$ being the Leray projection operator and thanks to (1.8), (A.1), and (A.2), we have

$$
\left\|\partial_{t} v\left(t_{1}\right)\right\|_{\dot{B}_{2,1}^{3 / 2}} \lesssim\left\|v\left(t_{1}\right)\right\|_{\dot{B}_{2,1}^{7 / 2}}+\left\|v\left(t_{1}\right)\right\|_{\dot{B}_{2,1}^{3 / 2}}\left\|v\left(t_{1}\right)\right\|_{\dot{B}_{2,1}^{5 / 2}} \lesssim\left\|u_{0}\right\|_{\dot{B}_{2,1}^{1 / 2}}
$$

and

$$
\begin{aligned}
& \left\|\partial_{t}(v \cdot \nabla v)\right\|_{L^{1}\left(\left[t_{1},+\infty\right) ; \dot{B}_{2,1}^{3 / 2}\right)} \lesssim\left\|\partial_{t} v\right\|_{L^{2}\left(\left[t_{1},+\infty\right) ; \dot{B}_{2,1}^{3 / 2}\right)}\|\nabla v\|_{L^{2}\left(\left[t_{1},+\infty\right) ; \dot{B}_{2,1}^{3 / 2}\right)} \\
& \quad+\|v\|_{L^{\infty}\left(\left[t_{1},+\infty\right) ; \dot{B}_{2,1}^{3 / 2}\right)}\left\|\nabla \partial_{t} v\right\|_{L^{1}\left(\left[t_{1},+\infty\right) ; \dot{B}_{2,1}^{3 / 2}\right)} \lesssim\left\|u_{0}\right\|_{\dot{B}_{2,1}^{1 / 2}}
\end{aligned}
$$

Plugging the above estimates into (A.3) gives (5.3) for $s_{2}=3 / 2$, which together (A.2) yields (5.3). This completes the proof of the proposition. 


\section{References}

1. AвIDI, H.: Équation de Navier-Stokes avec densité et viscosité variables dans l'espace critique. Rev. Mat. Iberoam. 23(2), 537-586 (2007)

2. AвIDI, H., Gui, G., Zhang, P.: On the decay and stability to global solutions of the 3-D inhomogeneous Navier-Stokes equations. Commun. Pure. Appl. Math. 64(6), 832-881 (2011)

3. Abidi, H., Paicu, M.: Existence globale pour un fluide inhomogéne. Ann. Inst. Fourier (Grenoble) 57, 883-917 (2007)

4. Bahouri, H., Chemin, J.Y., Danchin, R.: Fourier Analysis and Nonlinear Partial Differential Equations. Grundlehren der mathematischen Wissenschaften, vol. 343. Springer, Berlin, 2011

5. Bony, J.M.: Calcul symbolique et propagation des singularités pour les quations aux drivées partielles non linéaires. Ann. Sci. École Norm. Sup. 14(4), 209-246 (1981)

6. Chemin, J.Y.: Théorémes d'unicité pour le systéme de Navier-Stokes tridimensionnel. J. Anal. Math. 77, 27-50 (1999)

7. Chemin, J.Y., Gallagher, I.: On the global wellposedness of the 3-D Navier-Stokes equations with large initial data. Ann. Sci. École Norm. Sup. 39, 679-698 (2006)

8. Chemin, J.Y., Lerner, N.: Flot de champs de vecteurs non lipschitziens et équations de Navier-Stokes. J. Differ. Equ. 121, 314-328 (1995)

9. Danchin, R.: Density-dependent incompressible viscous fluids in critical spaces. Proc. R. Soc. Edin. Sect. A 133, 1311-1334 (2003)

10. DANChin, R.: Estimates in Besov spaces for transport and transport-diffusion equations with almost Lipschitz coefficients. Rev. Mat. Iberoam. 21(3), 861-886 (2005)

11. Danchin, R.: Local and global well-posedness results for flows of inhomogeneous viscous fluids. Adv. Differ. Equ. 9, 353-386 (2004)

12. Danchin, R.: The inviscid limit for density-dependent incompressible fluids. Anneles de la Faculté de Sciences des Toulouse Sér. 6 15, 637-688 (2006)

13. Danchin, R.: Well-posedness in critical cpaces for barotropic viscous fluids with truly not constant density. Commun. Partial. Differ. Equ. 32, 1373-1397 (2007)

14. Desjardins, B.: Regularity results for two-dimensional flows of multiphase viscous fluids. Arch. Ration. Mech. Anal. 137, 135-158 (1997)

15. DiPerna, R.J., Lions, P.L.: Equations différentielles ordinaires et équations de transport avec des coefficients irréguliers. In: Séminaire EDP 1988-1989. Ecole Polytechnique, Palaiseau, 1989

16. Fujita, H., Kato, T.: On the Navier-Stokes initial value problem I. Arch. Ration. Mech. Anal. 16, 269-315 (1964)

17. Gui, G., Zhang, P.: Global Smooth Solutions to the 2-D Inhomogeneous Navier-Stokes Equations with Variable Viscosity. Chin. Ann. Math. Ser. B 5, 607-630 (2009)

18. Kazhikov, A.V.: Solvability of the initial-boundary value problem for the equations of the motion of an inhomogeneous viscous incompressible fluid. (Russian). Dokl. Akad. Nauk SSSR 216, 1008-1010 (1974)

19. LADYŽENSKAJA, O.A., SOlONNIKOV, V.A.: The unique solvability of an initial-boundary value problem for viscous incompressible inhomogeneous fluids. (Russian) Boundary value problems of mathematical physics, and related questions of the theory of functions, 8, Zap. Naučn. Sem. Leningrad. Otdel. Mat. Inst. Steklov. (LOMI) 52, 52-109, 218-219 (1975)

20. Lions, P.L.: Mathematical Topics in Fluid Mechanics. Vol. 1. Incompressible models. Oxford Lecture Series in Mathematics and its Applications, Vol. 3. Oxford Science Publications. The Clarendon Press, Oxford University Press, New York, 1996

21. Peetre, J.: New Thoughts on Besov Spaces. Duke University Mathematical Series 1. Duke University, Durham, 1976

22. Triebel, H.: Theory of Function Spaces. Monograph in Mathematics, Vol. 78. Birkhauser Verlag, Basel, 1983 
23. Zhang, P.: Global smooth solutions to the 2-D nonhomogeneous Navier-Stokes equations. Int. Math. Res. Not. IMRN 2008, Art. ID rnn 098 (2008)

Département de Mathématiques,

Faculté des Sciences de Tunis Campus Universitaire Tunis, 2092 Tunisia.

e-mail: habidi@univ-evry.fr

and

Department of Mathematics,

Jiangsu University,

Zhenjiang 212013, People's Republic of China

and

The Institute of Mathematical Sciences,

The Chinese University of Hong Kong,

Shatin, N.T., Hong Kong.

e-mail: glgui@amss.ac.cn

and

Academy of Mathematics \& Systems Science and Hua Loo-Keng Key Laboratory of Mathematics,

Chinese Academy of Sciences,

Beijing 100190,

People's Republic of China.

e-mail: zp@amss.ac.cn

(Received November 18, 2010 / Accepted September 12, 2011)

Published online October 13, 2011 - (C) Springer-Verlag (2011) 